IZA DP No. 8104

Experimental Games on Networks:

Underpinnings of Behavior and Equilibrium Selection

Gary Charness

Francesco Feri

Miguel A. Meléndez-Jiménez

Matthias Sutter

April 2014 


\title{
Experimental Games on Networks: Underpinnings of Behavior and Equilibrium Selection
}

\author{
Gary Charness \\ University of California at Santa Barbara, \\ IZA and CESifo \\ Francesco Feri \\ Royal Holloway University of London \\ and University of Trieste
}
Miguel A. Meléndez-Jiménez
Universidad de Málaga

Matthias Sutter

European University Institute Florence, University of Innsbruck, University of Cologne and IZA

\author{
Discussion Paper No. 8104 \\ April 2014
}

\author{
IZA \\ P.O. Box 7240 \\ 53072 Bonn \\ Germany \\ Phone: +49-228-3894-0 \\ Fax: +49-228-3894-180 \\ E-mail: iza@iza.org
}

\begin{abstract}
Any opinions expressed here are those of the author(s) and not those of IZA. Research published in this series may include views on policy, but the institute itself takes no institutional policy positions. The IZA research network is committed to the IZA Guiding Principles of Research Integrity.

The Institute for the Study of Labor (IZA) in Bonn is a local and virtual international research center and a place of communication between science, politics and business. IZA is an independent nonprofit organization supported by Deutsche Post Foundation. The center is associated with the University of Bonn and offers a stimulating research environment through its international network, workshops and conferences, data service, project support, research visits and doctoral program. IZA engages in (i) original and internationally competitive research in all fields of labor economics, (ii) development of policy concepts, and (iii) dissemination of research results and concepts to the interested public.
\end{abstract}

IZA Discussion Papers often represent preliminary work and are circulated to encourage discussion. Citation of such a paper should account for its provisional character. A revised version may be available directly from the author. 


\section{ABSTRACT}

\section{Experimental Games on Networks: Underpinnings of Behavior and Equilibrium Selection*}

In this paper, we describe a series of laboratory experiments that implement specific examples of a more general network structure and we examine equilibrium selection. Specifically, actions are either strategic substitutes or strategic complements, and participants have either complete or incomplete information about the structure of a random network. Since economic environments typically have a considerable degree of complementarity or substitutability, this framework applies to a wide variety of settings. The degree of equilibrium play is striking, in particular with incomplete information. Behavior closely resembles the theoretical equilibrium whenever this is unique; when there are multiple equilibria, general features of networks, such as connectivity, clustering, and the degree of the players, help to predict informed behavior in the lab. People appear to be strongly attracted to maximizing aggregate payoffs (social efficiency), but there are forces that moderate this attraction: 1) people seem content with (in the aggregate) capturing only the lion's share of the efficient profits in exchange for reduced exposure to loss, and 2) uncertainty about the network structure makes it considerably more difficult to coordinate on a demanding, but efficient, equilibrium that is typically implemented with complete information.

JEL Classification: C71, C91, D03, D85

Keywords: random networks, incomplete information, connectivity, clustering, strategic substitutes, strategic complements, experiment

Corresponding author:

Matthias Sutter

European University Institute

Department of Economics

Via della Piazzuola 43

50133 Florence

Italy

E-mail: matthias.sutter@eui.eu

\footnotetext{
* We thank Juan Carrillo, Matthew Jackson, Fernando Vega-Redondo, four referees, and seminar participants at Stanford University, Chapman University, University of Amsterdam (CREED), University of Lyon, University of Bologna, Royal Holloway University of London, University of the Basque Country, 2011 EWEBE conference, the 2012 Royal Economic Society Meeting, and the 2012 CESifo conference in Munich for valuable comments. We are grateful for financial support from the University of Innsbruck. Miguel A. Meléndez-Jiménez acknowledges financial support from the Spanish Ministry of Economy and Competitiveness through project ECO2011-26996, and from the Regional Government of Andalusia through projects SEJ2009-4794 and SEJ2011-8065.
} 


\section{Introduction}

Social networks are a prominent feature of the economic landscape. A network is a nonmarket institution, but has important market-like characteristics. In a sense it can be considered to be an intermediate case between bilateral bargaining and matching in a large centralized market. Network structure affects choices in a wide variety of environments and network analysis has been applied to many important environments. ${ }^{1}$ Examples include systems compatibility (Katz and Shapiro 1994), airline route design (Hendricks, Piccione and Tan 1995), matching markets (Gale and Shapley 1962, Kelso and Crawford 1982, Roth 1984, Crawford and Rochford 1986, Roth and Sotomayor 1989), bargaining (Kranton and Minehart 2001), and friendship (Currarini, Jackson and Pin, 2009). Network analysis is also useful for job search and labor-market issues, since workers frequently find jobs through personal contacts and employers value the additional enforcement channel available through these personal intermediaries (Montgomery 1991, Calvó-Armengol 2004, Calvó-Armengol and Jackson 2004, 2007).

A growing empirical literature has documented the effects of social networks on behavior; the information gleaned from these has motivated theoretical work. Since social networks are so prevalent in economic settings, modeling these networks is essential in order to understand how network structure affects behavior. However, it is very difficult (if not impossible) to cleanly test theoretical predictions using field data, since there are many confounding features in the environment. ${ }^{2}$ In this respect, controlled laboratory experiments are often viewed as the ideal tool for qualitatively testing theory (e.g., Falk and Heckman, 2009).

In this paper, we describe a series of laboratory experiments that implement specific examples of a more general network structure. Our starting point is the model in Galeotti, Goyal, Jackson, Vega-Redondo, and Yariv (2010), which considers environments in which the agents' actions are either strategic complements or substitutes. Economic environments typically have a considerable degree of complementarity or substitutability, so that this notion applies to a wide variety of economic settings and includes many game-theoretic applications in the network literature. Strategic complements arise when the marginal benefit that an individual obtains from

\footnotetext{
${ }^{1}$ Jackson (2010, p. 512) states that network structure "influences patterns of decisions regarding education, career, hobbies, criminal activity, and even participation in micro-finance.” For an exhaustive review of social and economic networks, with particular attention to theoretical models, see Jackson (2008).

${ }^{2}$ Typical problems with field data are the use of idiosyncratic data sets, multiple simultaneous influences, and the issue of measurement error.
} 
choosing an action is greater when more of her neighbors do the same. An example of strategic complements is human capital investment, whereby one's own investment is marginally more beneficial if others also make this investment. Strategic substitutes arise when the marginal benefit that an individual obtains from choosing an action is greater when fewer of her neighbors do the same. An example is choosing routes to avoid congested roads, since one certainly receives a greater (marginal) benefit from choosing a route that has been chosen less by others.

Our study is also one of the first to experimentally consider an environment in which the agents are uncertain about the precise network structure. ${ }^{3}$ This enhances the applicability and the external validity of our experiment, as there are many economic situations in which individuals have a good sense of the number of other people with whom they are interacting in some form of network, but know neither the identity of these others nor how these others are connected to still others. As examples for such situations, Galeotti et alii (2010) mention choosing which languages to study before embarking on a career in diplomacy, researchers choosing software based on compatibility, and choosing whether to receive a vaccination.

A critical problem for network theory is that even simple games have multiple equilibria, so that a great variety of outcomes are consistent with theoretical analysis. This naturally limits the predictive power of the theory and the scope of policy recommendations, since multiple equilibria make it difficult-to-impossible to offer definitive advice regarding how such labor markets, search markets, etc. should be organized. To make meaningful policy recommendations, it is crucial to determine which equilibrium is likely to occur. Consequently, a central goal in network analysis is to refine the set of equilibria to be able to make better predictions about the likely outcomes. As Galeotti et alii (2010) state, in some cases with networks games "much of the equilibrium multiplicity that arises under complete information is no longer sustainable under incomplete information.”4 Another method for examining equilibrium selection is through experimental testing. This is our approach, as experimental work can provide empirical information regarding which of the multiple equilibria tends to actually prevail behaviorally and may even lead to clear insights ex post.

In our first set of experiments, we consider a specific environment that includes three

\footnotetext{
${ }^{3}$ A previous paper that introduces uncertainty about the network structure is Berninghaus et alii (2002), which we describe in more detail in Section 2.

${ }^{4}$ They argue (p. 219) that the key insight is that "when players have limited information about the network they are unable to condition their behavior on its fine details and this leads to a significant simplification and sharpening of equilibrium predictions”.
} 
different five-person networks. In the case of complete information, each person knows the network structure (which of the three networks is in play) and the node to which she has been assigned. In contrast, with incomplete information each person only knows her degree - i.e., the number of connections to others - and the probability (a treatment variable) that each of the three possible networks has been randomly drawn. The results provide striking support for the theoretical predictions. Participants are largely active (which could be interpreted as purchasing a particular good) or inactive (not purchasing) in the network according to (one of) the theoretical prediction(s). In all scenarios, the modal behavior by every individual is consistent with the observed equilibrium outcome, and the overall rate of such equilibrium play is quite high.

In the simpler case of complete information, we find strong evidence that groups play a particular equilibrium, even though there are at least three potential equilibria in all cases with strategic substitutes and two potential equilibria in one case with strategic complements. There are two kinds of equilibria: efficient ones that yield the highest aggregate payoff for the network, and more secure ones (Van Huyck, Battalio and Bail, 1990) in which (many or all) players choose the maximin action. In the experiment, behavior that is highly consistent with the same particular equilibrium is observed in each and every independent group. With strategic substitutes, the equilibrium played is not the efficient one, but in a certain sense is 'more secure', as deviations from this equilibrium are less harmful than deviations from the efficient one(s). In other words, there is a trade-off between efficiency and the cost of a mistake, since the efficient equilibrium results in a higher cost for agents' errors. With strategic complements, the efficient equilibrium is selected. Remarkably, the selected equilibrium is played qualitatively for every node and also quantitatively (within 10 percentage points of the extreme point-prediction) for most nodes, for both strategic substitutes and strategic complements.

With incomplete information, one knows only one's degree and the probability of each network being selected, so we do not distinguish amongst positions with the same degree. For this scenario, Galeotti et alii (2010) show that, under a wide class of games where agents' choices are either strategic complements or strategic substitutes to those of their neighbors, ${ }^{5}$ there is an equilibrium in which players use monotone (threshold) strategies: In the game of strategic

\footnotetext{
${ }^{5}$ Galeotti et alli (2010) study symmetric Bayes-Nash equilibria. They mainly focus on games that satisfy what they call Property A: Adding a link to a neighbor who is inactive is payoff equivalent to not having an additional neighbor. Note that this restriction excludes from their (and our) scope some economic setups (like the study of peer effects where the average actions of a reference group are considered).
} 
substitutes (complements) players are active if their degree is below (above) a certain threshold and inactive otherwise. They also consider the effects of connectivity, which is the extent to which the nodes of the network are connected. ${ }^{6}$ When the connectivity of the network increases, in the case of strategic substitutes (complements) the maximum (minimum) degree's value for which an agent is active increases (decreases). Our data support the qualitative predictions of the model in both cases. We observe that participants do use threshold strategies, and the frequency of active players increases with connectivity. In scenarios where incomplete information induces a unique equilibrium, we see that participants make the choice that is consistent with this equilibrium an overwhelming majority of the time. Our experiment demonstrates that the theory in Galeotti et alii (2010) does remarkably well in predicting behavior in our initial networks in the incomplete-information scenario.

However, our initial networks were not ideal for the purpose of studying equilibrium selection, and questions remained concerning the underpinnings. Hence, in order to study the issue of equilibrium selection in more detail, we conducted new treatments with another set of five-person networks (our second set of experiments) and with a set of 20-person networks (our third set of experiments). In the second set of experiments, we focus on strategic complements both with complete and incomplete information. In the third, we consider strategic complements and incomplete information. ${ }^{7}$ In the new sets of experiments, we can test if the connectivity and clustering of the network affect equilibrium selection. Clustering refers to the increased propensity of people to be linked with one another if they have another neighbor in common. ${ }^{8}$

In all the scenarios considered in the new treatments we have an efficient equilibrium and an inefficient but secure equilibrium. An increase of the connectivity raises the payoffs of the efficient equilibrium, while the payoffs of the other equilibrium remain unaltered. Thus we conjecture that connectivity fosters efficiency. ${ }^{9}$ A similar effect is expected for degree, since a player with a higher degree can (potentially) achieve a higher payoff by selecting the efficient

\footnotetext{
${ }^{6}$ Formally, a (random) network is more connected than another one when the neighbors' degree distribution of a network first-order stochastically dominates that of the other. For all of our networks, a higher (expected) average degree is linked with higher connectivity.

${ }^{7}$ We only consider complements in this environment because this offers a multiplicity of equilibria in all scenarios. We only consider incomplete information in the large-network case because, with complete information, the number of equilibria would be very large, and each player's strategy would have a huge number (20) of components.

${ }^{8}$ One simple measure of the degree of clustering of a network is the ratio between the number of closed triples and the number of potential closed triples. By a cluster or clique we refer to a fully-connected triple of nodes.

${ }^{9}$ Intuitively, the higher the payoff of an equilibrium, the more likely it is to be played, ceteris paribus. For experimental evidence see Brandts and Cooper (2006).
} 
action; therefore, we surmise that players with higher degree are more likely to be active. Finally, we also suspected that the degree of clustering in the network would have an important influence on behavior and equilibrium selection, since an increased level of clustering reduces the independence of the equilibrium strategies of the neighbors. ${ }^{10}$ Previous experimental evidence suggests that, in the context of coordination games played on networks, higher clustering results in the efficient equilibrium being played more frequently (see Berninghaus, Ehrhart and Keser, 1998, 2002, and Cassar, 2007, which are discussed in Section 3). Thus, we conjectured that clustering also fosters efficiency and is therefore important for equilibrium selection. The data gathered from the second and third sets of experiments support all these three behavioral hypotheses in small and (relatively) larger network environments, respectively.

We also consider a puzzle from the first set of experiments concerning the effects of uncertainty on the selection of the efficient equilibrium with strategic complements. To test whether the introduction of uncertainty per se can drive the selection of the inefficient equilibrium, we ran an additional treatment with a minimal degree of uncertainty. The results suggest that there is indeed an important effect of uncertainty per se on behavior in network games, as it appears to play a role in driving play to the most secure equilibrium.

People largely behave in accordance with some simple principles and generally make very sensible choices in complex environments. First, behavior closely resembles the theoretical equilibrium when this is unique. Second, when there are multiple equilibria, there are general features of networks, such as connectivity, clustering, and the degree of the players, that predict informed behavior in the lab. Third, our evidence reveals some specific patterns. With complete information and substitutes, people select an equilibrium that delivers nearly as much overall profit as the optimal one; with complete information and complements they select the efficient one. As discussed later, this difference can be explained by looking at the relation between efficiency and private incentives. The inactive equilibrium is rarely played when there is another equilibrium with activity; this is consistent with experimental work in which payoff dominance is a key consideration for equilibrium selection (Charness, 2000; Brandts and Cooper, 2006).

\footnotetext{
${ }^{10}$ In equilibrium the strategies of individuals are best responses to the strategies of the neighbors. Therefore, the equilibrium strategies of two players (linked or not) display more correlation as the number of their common neighbors increases (perfect correlation with the same set of neighbors). Thus, if two players A and B are neighbors in a (larger) network, and we increase the clustering by adding a new link between A and a neighbor of B (say C), then the correlation between the equilibrium strategies of $\mathrm{A}$ and $\mathrm{B}$ is higher (since $\mathrm{C}$ is now a common neighbor).
} 
And yet there are some moderating forces on the attraction to efficiency: 1) people seem content with only the lion's share of the efficient profits in exchange for greater security, and 2) uncertainty about the network structure makes it considerably more difficult to coordinate on a demanding, but efficient, equilibrium that is typically implemented with complete information. We believe that our findings, while certainly not a full characterization, nevertheless offer considerable predictive power for behavior and equilibrium selection in games on networks.

The remainder of the paper is organized as follows. We discuss the relevant literature in section 2, and describe the experimental design and implementation in section 3 . Our experimental results are given in section 4, and we offer a discussion of our results and their implications in section 5. We conclude in section 6, and propositions and proofs regarding our networks are given in the Appendix.

\section{Literature review}

In this section we review related work. We refer the interested reader to Jackson (2008) for a comprehensive overview of theoretical work and applications of social and economic networks.

Regarding theoretical work, a handful of papers show that the outcomes of games in general depend on the specific network structures, when there are either strategic substitutes or complements and either complete or incomplete information. ${ }^{11}$ Galeotti et alii (2010) was the starting point for our experimental design and our initial task was to adapt this theory to a distilled selection of networks that represented their framework.

Overall, there is relatively little research in experimental economics on network games, particularly given the wealth of theoretical contributions in this area. ${ }^{12}$ Here we restrict our discussion of the literature in experimental economics to designs with exogenous networks (where the participants have no control of the network structure), as in our own environment. ${ }^{13,14}$

\footnotetext{
${ }^{11}$ For the complete-information case, see for example Ballester, Calvó-Armengol and Zenou (2006), Bramoullé and Kranton (2007), Goyal and Moraga-Gonzalez (2001), and Calvó-Armengol and Jackson (2004). For the incompleteinformation case, see Jackson and Yariv (2005), Sundararajan (2006), and Galeotti and Vega-Redondo (2011).

${ }^{12}$ Researchers in sociology have long been interested in studying networks in experiments (see the seminal studies by Stolte and Emerson, 1977, or Cook and Emerson, 1978; see also surveys of Willer, 1999, or Burt, 2000). Note, however, that sociologists have been in particular interested in studying the exercise of power in networks, something with which the literature in experimental economics has not yet been concerned.

${ }^{13}$ Thus, we do not consider the issue of how networks were formed, but simply presume that the links are already in place due to some relationships that have (or had) value, and that the cost of endogenous change is prohibitive. In a sense, the study of agents' behavior under exogenous networks is a simplification of economic situations in which
} 
Some research has examined the consequences of network structure on equilibrium selection in coordination games, which is relevant for our settings with a multiplicity of equilibria (game of strategic complements). Keser, Ehrhart and Berninghaus (1998) use a 3person coordination game; in one treatment, each participant is connected to two neighbors on an 8-player circle, while in the other treatment, people play within closed 3-person groups. The 3person group quickly coordinates on the payoff-dominant equilibrium while the circular group eventually coordinates on the risk-dominant equilibrium.

Berninghaus, Ehrhart and Keser (2002) extend the design of Keser et alii (1998) to a more general framework, in order to gain more insight on the impact of neighborhood sizes and structures on equilibrium selection. Specifically, they add a network of 16 players arranged in a lattice with four neighbors each and a network of 16 players arranged in a circle, each linked to the four closest players. A comparison between the 16-player networks shows more coordination on the efficient equilibrium with the circle. The main differences between their design and ours are: (i) they focus on homogeneous and symmetric networks, whereas we consider heterogeneous networks, (ii) in their design adding a link to a neighbor who chooses inactivity is not payoff equivalent to not having an additional neighbor, ${ }^{15}$ and (iii) they consider a setup where subjects only know their degree (number of neighbors), but are uninformed about any other feature of the network, including the (random) generating process of the network. Our design allows participants to possibly perform more sophisticated calculations: They can potentially form (Bayesian) beliefs (as in Galeotti et alii, 2010) and calculate the equilibria.

Cassar (2007) compares convergence to equilibrium in three different network structures: a local-interaction network (similar to that in Berninghaus et alii, 2002), a random network, and a "small-world" network (each link in the local-interaction network has a probability of being rewired to a 'short cut' of a chord across the circle). Participants converge to the efficient equilibrium of the coordination game in the small-world network, but less so in the others.

networks adjust very slowly, whereas interaction among the agents located in the network can be very frequent (e.g., in housing neighborhoods, networks of co-workers, insurance networks in developing countries, etc.).

14 There are other experiments on networks in other environments, including buyer-seller networks (Charness, Corominas-Bosch, and Fréchette 2007), the prisoner's dilemma (Riedl and Ule 2002; Kirchkamp and Nagel 2007), and endogenous networks (Falk and Kosfeld 2003; Deck and Johnson 2004; Callander and Plott 2005; Berninghaus, Ehrhart, and Ott 2006; Berninghaus, Ehrhart, Ott, and Vogt 2007).

${ }^{15}$ This property is key to most of the results in Galeotti et alii (2010). 
Cassar's (2007) results suggest that high network clustering and short network path length increase the probability with which agents coordinate on the payoff-dominant equilibrium. ${ }^{16}$

Within the scope of experimental games of strategic substitutes played in networks, the most closely related paper to our study is Rosenkranz and Weitzel (2012). They consider the public-goods game on networks studied theoretically by Bramoulle and Kranton (2007), which is similar to our environments with strategic substitutes and complete information. The main differences are that in their game people can choose partial activity and that their benefit function is strictly increasing in the neighbors' actions. They study 4-player networks with repeated matching. The network and one's position within the network are fixed. Individuals find it difficult to coordinate on equilibria, but there is more coordination on equilibria in which each player is either fully active or inactive, and equilibrium play is more prevalent in networks with high (low) average degree and low (high) centrality. When convergence occurs, this involves a fully inactive player connected to at least two fully active players, ${ }^{17}$ which is related to our finding that an inefficient but more secure equilibrium is mostly played with complete information and substitutes in all three networks. They also find a negative correlation between individual contributions and degree, reflecting the idea that players with lower degree are the more 'active' ones in the network.

Other experimental studies that consider public-good games played in networks are Fatas, Meléndez-Jiménez and Solaz (2010) and Choi, Gale, Kariv and Palfrey (2011). ${ }^{18}$ Fatas et alii (2010) consider network effects primarily in relation to the voluntary-contribution mechanism. Four-person groups play repeatedly a standard VCM in four different network structures: the line, the circle, the star, and the complete network. In their (fixed-matching) setup, the network only determines the information about past behavior that each player receives (i.e., information about others' past contributions is transmitted if and only if there is a direct link between the parties). Therefore, an agent's payoffs depend on the choices made by all the population, rather

\footnotetext{
${ }^{16}$ Charness and Jackson (2007) frame a Stag Hunt as the choice of adding a link between two players in a preexisting network, where this link can be added by either mutual or unilateral consent. Whether the payoff-dominant or the risk-dominant equilibrium prevails depends on the degree of consent required. Boun My, Willinger, and Ziegelmeyer (2006) and Corbae and Duffy (2008) also study experiments on coordination games in networks.

${ }^{17}$ This type of equilibrium is the only stable one in Bramoulle and Kranton (2007) with a Nash tâtonnement process.

${ }^{18}$ See also Carpenter (2007), which mainly considers the issue of group size in the VCM, but also has treatments in which people are only allowed to punish their closest neighbors. Relative to not punishing at all, both the possibility of monitoring either half or all of the group yields significantly more contributions, and the possibility of punishing only a single player elicits significantly fewer contributions. In a more recent paper, Carpenter et alii (2012) study the effects of punishment in VCM played in networks.
} 
than on the choices made in his local network, as in this paper. They find that contributions are affected by the network structure, with 30-40 percent higher contributions with the complete network and the star than with the line and the circle. There is at least one person with a degree of three in each of the networks with higher contributions; such a person learns the contribution of every other player and every other player observes their choice, with this being common information. The degree of an individual does not appear to affect contributions, however.

Choi et alii (2011) study directed networks of three players in the lab. In each of three stages, each player must decide whether to irreversibly contribute his endowment to a public good. At each stage, players can only observe the past decisions of neighbors in the network. The public good is provided if and only if at least two players have contributed by the end of the third stage. Each player earns his unit of endowment (if not contributed) plus two units (if the public good has been provided). There are inefficient equilibria (no one contributes) and efficient equilibria (two agents contribute). Participants who do not observe others but are observed by others tend to contribute early, while those who observe others tend to delay their contributions. In their paper, the network only matters through the information received in the three stages. Furthermore, payoffs depend on own and global behavior, rather than depending only on own and neighbors' behavior. Moreover, our networks are larger and have a variety of structures.

Kearns et alii (2006, 2009) conduct experiments where players have a collective goal, studying how the capacity to achieve this goal depends on the network structure of communication. Kearns et alii (2006) consider a game of substitutes in which all players receive exactly the same payoff, which depends only on whether the collective goal is achieved or not; it is more difficult to achieve success with networks generated by preferential attachment than with either 'small-world' networks or networks based on cyclical structures. ${ }^{19}$ Kearns et alii (2009) examine a game of complements, where the entire group aims to coordinate (vote) on a choice, and where there is heterogeneity in preferences. Their findings suggest that some network structures better promote coordination, and that the presence of individuals, with extreme views or the awareness of opposing incentives, reliably improve collective performance. ${ }^{20}$ In contrast to these studies, the key aspect of our design is that earnings and optimal strategies are directly

\footnotetext{
${ }^{19}$ Preferential attachment is a stochastic process of network generation, in which the more connected a node is, the more likely that it will have more new links as the network is formed incrementally.

${ }^{20}$ See also the recent study by Choi and Lee (2014), which investigates how the interaction between the network structure of pre-play communication and the length of such communication affects outcome and behavior in a similar coordination context.
} 
related to network features such as the degree; also, in our case players only care about their own choices and those of their neighbors. Additionally, we present a framework where one choice is secure and the other one is efficient but 'risky'. Hence, we have multiplicity of equilibria of very different natures, which renders the equilibrium-selection problem a crucial issue.

In sum, our experiments can be seen as venturing into some new realms. We contrast strategic complements and strategic substitutes, considering both complete and incomplete information concerning aspects of the network structure.

\section{Experimental design}

In the experiments in this paper we focus on the two specific games that Galeotti et alii (2010) use to introduce and motivate their results, which we now briefly summarize. Consider a player who can choose between being active (e.g., buying a product) or inactive (e.g., not buying the product). The player is located in a position within a network and her payoff depends both on her choice and on the choices of her neighbors.

- With strategic substitutes, a player earns 100 if either she or at least one of her neighbors is active, and earns 0 otherwise. Being active costs 50 , while inactivity is costless.

- With strategic complements, if a player is inactive, she earns 50 and, if she is active she earns 33.33 times the number of neighbors who are active. ${ }^{21}$

In each of our two games, there is a choice that provides the subject a fixed payoff of 50 , independently of the degree and of the neighbors' decision. This choice is active in the game of strategic substitutes, whereas it is inactive in the game of strategic complements. Note that this choice is indeed the maximin action (pure strategy), since it provides a player the largest payoff in the worst possible outcome. In this sense, this choice is secure (see Von Neumann and Morgenstern, 1944, 1972). Following Van Huyck, Battalio, and Beil (1990), we denote an equilibrium in which all players choose the secure (maximin) action as a secure equilibrium. When there is no such equilibrium, we refer to the equilibrium with the highest number of players choosing the secure action as the most secure equilibrium. On the other hand, we say that an equilibrium is efficient if it provides the maximum feasible aggregate payoff.

As we shall see below, when there are multiple equilibria, the efficient and the (most) secure ones differ. In these cases, although the secure equilibrium is inefficient, it typically has

\footnotetext{
${ }^{21}$ In our experimental design, to avoid losses in the case of strategic complements we have added 50 to all the payoffs as compared to the original game used by Galeotti et alii (2010).
} 
very good properties in terms of risk-dominance and (stochastic) stability. In our multi-player games, we cannot invoke the Harsanyi and Selten (1988) concept of risk-dominance, but we can rely on the concept of ordinal generalized-risk dominance, proposed by Peski (2010). Indeed, Peski (2010) shows that an ordinal generalized-risk dominant profile is stochastically stable under a class of evolutionary dynamics. In our treatments, when there exists an ordinal generalized risk-dominant equilibrium, it is always the (most) secure equilibrium (see Online Supplement D). ${ }^{22}$ Thus, in many of our treatments, the equilibrium-selection problem between the efficient and the secure equilibrium reflects the traditional trade-off between efficiency and risk dominance.

All networks used in our experiments are shown in Figure 1:

[Figure 1]

\subsection{Experiment 1}

In this study we used the three networks displayed in the top panel: The Orange, Green and Purple networks. With incomplete information, $p$ is the probability that the Orange network is in force, with each of the other two networks being selected with probability (1- $p$ )/2. Note that, since the Orange network has a higher connectivity than the other two ones (the Orange network is the Green network with the link BD added and is also the Purple network with the link CD added), we modulate the connectivity by parameter $p$. We had 12 sessions with 20 participants in each. There were two sessions in each of the six treatments below.

- strategic substitutes with complete information;

- strategic complements with complete information;

- $\quad$ strategic substitutes with incomplete information and $p=0.2$;

- $\quad$ strategic substitutes with incomplete information and $p=0.8$;

- $\quad$ strategic complements with incomplete information and $p=0.2$;

- $\quad$ strategic complements with incomplete information and $p=0.8$.

In each session, the 20 participants were split randomly into two matching groups of 10 subjects, and this was common information. In each of 40 periods (plus five unpaid trial periods,

\footnotetext{
${ }^{22}$ There exists an ordinal generalized-risk dominant equilibrium in all the treatments of strategic complements of Experiments 1 and 2 (both with complete and incomplete information). In the case of strategic substitutes, when there are multiple equilibria (complete information scenario), only in one of the networks does there exist a generalized risk-dominant equilibrium. However, in all the networks the most secure equilibrium is stochastically stable under a class of evolutionary dynamics, as shown by Boncinelli and Pin (2012) - see Section 4.1 for details.
} 
with the same feedback structure as below), the members of a matching group were randomly assigned to groups of five subjects who played the stage game of a given treatment.

The experimental instructions are provided in Online Supplement C. ${ }^{23}$ In treatments with complete information, participants were always informed at the beginning of a period about the chosen network (which was re-drawn each period, each network being equally likely) and the participant's position in it. At the end of a period, each person received feedback about her neighbors' decisions and the payoff resulting from her choice and those of her neighbors. Before a new period began, participants also received the respective feedback for all prior periods. In treatments with incomplete information, subjects were informed about their degree at the beginning of a period. At the end of the period, each person received information about the actual network that was in effect, her position in it, the number of her neighbors who chose to be active, and the payoff resulting from her choice and those of her neighbors. Payoffs were given in ECUs (Experimental Currency Units), with $20 \mathrm{ECU}=1$ Euro. $^{24}$

Based on the equilibrium analysis reported in the Appendix, we summarize the purestrategy equilibrium predictions for each treatment of Experiment 1 in Table $1 .^{25}$ For the complete-information scenario, we report the Nash equilibria, and for the incompleteinformation scenario we report the symmetric Bayes-Nash equilibria. ${ }^{26}$

\section{[Table 1]}

With complete information and strategic substitutes, all three networks have equilibria in in which two nodes are active and equilibria in which three nodes are active. The former are efficient, and the latter are the most secure ones according to the definition given above. In the case of strategic complements, we have a unique equilibrium in the green and purple networks, where all individuals are inactive; and there are two equilibria in the orange network: In addition

\footnotetext{
${ }^{23}$ We only provide the instructions for “complete information - substitutes" and "incomplete information complements $-p=0.8$ ”. The remaining cases are analogous.

${ }^{24}$ Since behavior could potentially be affected by risk preferences, we also tested for these in Part 2 of the experiment, using two elicitation methods (see Charness and Gneezy, 2010). We find that the marginal effect of risk aversion on the probability of being active is almost always insignificant. At the beginning of the experiment it was announced that the experiment would consist of two independent parts. Part 1 was the network game. The instructions for Part 2 were only distributed after the end of Part 1 . The average payoff from the risk-preference task was 4.36 Euro. Note that participants had no information during Part 1 about the potential gains from the subsequent risk task, so that the size of the payoffs from the risk task could not affect behavior in Part 1.

${ }^{25}$ We refer readers interested in the mixed-strategy equilibria to the working-paper version, Charness et alii (2012).

${ }^{26}$ In the incomplete-information scenario, we focus on symmetric Bayes-Nash equilibria, (i.e., all players with the same degree choose the same strategy). Galeotti et alii (2010) restrict their main analysis also to symmetric BayesNash equilibria in order to clearly elicit the pure effects of networks on behavior and welfare, without any potential confounds that might arise from egalitarian concerns in case of asymmetric equilibria.
} 
to the (no-activity) secure equilibrium, there is an efficient equilibrium in which the clique formed by positions B, C and D is active.

With incomplete information, Galeotti et alii $(2010)^{27}$ show that the equilibria are defined by a threshold: In the case of strategic substitutes, those players with degree below (above) the threshold are active (inactive), and the threshold increases with connectivity. In the case of strategic complements, those players with degree above (below) the threshold are active (inactive) and the threshold decreases with connectivity. Thus, as depicted in Table 1, in the case of strategic substitutes, the theoretical prediction is that players with degree 1 (degree 3 ) are active (inactive) in both treatments, i.e., $p=0.2$ and $p=0.8$. Players with degree 2 are active only when $p=0.8$. With strategic complements, the theoretical prediction is that no one will be active when $p=0.2$, but that (in addition to the no-activity secure equilibrium) there is room for players with high degree (degrees 2 and 3 ) to be active in (an efficient) equilibrium when $p=0.8$.

Comparing across informational regimes (Table 1), we see that the equilibrium multiplicity with complete information and strategic substitutes is fully resolved with incomplete information, as well as with strategic complements and $p=0.2$ (but not $p=0.8$ ). Thus, we can study if people are behaviorally responsive to the different network positions and the levels of information they have when the incentives are either complements or substitutes.

We conducted this initial set of sessions at the University of Innsbruck in March of 2011, using the software zTree (Fischbacher 2007). A total of 240 undergraduate students from various academic disciplines were recruited with the help of ORSEE (Greiner 2004). No subject was allowed to participate in more than one session. On average, a session lasted about 80 minutes, with an average payoff of 16 Euro per subject (including a 5 Euro show-up fee).

After conducting these sessions and seeing the results, we added an extra treatment to the Experiment 1 setting. In an effort to understand the dramatically-different behavior with strategic complements according to whether the orange network is certain or only 80 percent likely, we also ran two sessions with our initial networks and with the likelihood of $p=0.95$ that the orange network being in force. The set of (pure-strategy) equilibria in this additional case coincides with the set of equilibria for $p=0.8$ : There is one equilibrium in which all degrees are inactive and

\footnotetext{
27 Another set of results presented in Galeotti et alii (2010) are related to mean preserving spread in degree distributions: keeping connectivity the same but allocating links differently. In a sense, Experiment 3 provides some evidences related to this issue, because in two treatments we preserve the degree distribution but change the network structure.
} 
another one in which players with degree 1 are inactive and players with degrees 2 and 3 are active. A total of 40 new subjects participated in these sessions.

\subsection{Experiment 2}

After observing our results for Study 1 and receiving helpful comments, we designed a new experiment to study the issue of equilibrium selection in the lab in more detail. We focus on strategic complements in the new treatments. ${ }^{28}$ The new treatments are:

- strategic complements with complete information;

- strategic complements with incomplete information and $p=0.2$;

- strategic complements with incomplete information and $p=0.8$.

In this experiment, we used the networks depicted in the middle panel of Figure 1: The Blue, Red and Brown networks. With incomplete information, the value for $p$ is the probability that the Blue network was in force, with each of the other two networks being selected with probability (1-p)/2. We created these three new networks by adding a link to each of the three initial networks used in Experiment 1 (with re-labeling to avoid visually-crossed lines). ${ }^{29}$ We note that the Red and Brown networks have the same average connectivity but different clustering, with a clustering coefficient of 0.5 (0) for the Red (Brown) network. The Blue network has higher average connectivity, but also has a higher clustering coefficient (of 0.6). ${ }^{30}$

Table 2 shows the theoretical predictions for the network scenarios in Experiment 2, which are derived in the equilibrium analysis reported in the Appendix.

[Table 2]

Note that all treatments share two equilibria, the secure one in which all subjects are inactive and the efficient one in which the maximum number of players who can (profitably) coordinate on activity do so. ${ }^{31}$ So this design allows us to study the equilibrium selection and to relate it to relevant network characteristics: connectivity, degree and clustering.

\footnotetext{
${ }^{28}$ This is so since, in the main context (incomplete information) analyzed in Galeotti et alii (2010), there are multiple equilibria with strategic complements, whereas they find a unique equilibrium in the case of substitutes. In the complete-information scenario in the new set of treatments, we also focus on the case of strategic complements.

${ }^{29}$ Specifically, we add the link CE to each of the networks, and then switch the labels of nodes D and E.

${ }^{30}$ The (global) clustering coefficient of a network is defined as three times the number of triangles (cliques) divided by the number of connected triples of vertices. A triangle is a trio of vertices, each of which is connected to both of the others, and a connected triple is a trio in which (at least) one of the vertices is connected to both of the others. Each triangle contributes to three connected triples of vertices (see Newman et alii 2001), ergo the factor of three.

31 The incomplete-information scenario presents an additional Bayes-Nash equilibrium in which only players with degree 3 are active when $p=0.8$. However this equilibrium is weak, since players with degree 2 are indifferent between being active or inactive (see the Appendix). It is an evanescent equilibrium since, in any dynamic setup, if
} 
A comparison between the $p=0.2$ and $p=0.8$ treatments lets us test the (behavioral) hypothesis that an increase in the connectivity increases the probability that the efficient equilibrium is played, since the higher the connectivity, the higher the payoffs with coordination on the efficient equilibrium, and so the more likely the efficient equilibrium is played. Similarly, we also conjecture that the degree of a subject increases the probability that she is active.

Regarding clustering, Keser et alii (1998) show that three-player cliques achieve more coordination than do 8-player circles (with zero clustering), despite the same average connectivity, ${ }^{32}$ and Berninghaus et alii (2002) find that there is more coordination on the efficient equilibrium in a (homogeneous) network of 16 players arranged in a circular structure and linked to their closest 4 neighbors (hence, with positive clustering) than in a 16-players lattice with the same average connectivity (with zero clustering). The results in Cassar (2007) reinforce the idea that clustering increases the probability that agents coordinate on the efficient equilibrium, ${ }^{33}$ and the experimental evidence supports the idea that small groups coordinate on the payoff-dominant equilibrium more than larger ones do (see, for instance, Van Huyck et alii, 1990). Thus, our (behavioral) hypothesis is that environments with higher clustering should support more coordination on the efficient outcome.

We conducted the sessions of Study 2 in Innsbruck in October of 2012. There were two sessions of each of the treatments with the new 5-person networks, and thus 40 people in each of these treatments, yielding a total of 120 new participants.

\subsection{Experiment 3}

We designed a new experiment using a more complex environment to confirm the results previously observed with respect to equilibrium selection. We use the three different 20-person networks shown in the bottom panel of Figure 1. We consider only strategic complements for the same reasons mentioned above (cf. Footnote 28). In this case, we additionally focus on the incomplete-information scenario because the strategy space in the case of complete information would be very large. Players know the network but not their position within the network. In each

\footnotetext{
one degree-2 player switches from inactive to active (also a best response), then all remaining players with degree 2 would have (strict) incentives to become active. Moreover, it is inefficient, as it is Pareto dominated by the (efficient and strict) equilibrium in which players with degrees 2 and 3 are active. For these reasons, and because it has no behavioral support in our data, we generally ignore this equilibrium in our analysis.

${ }^{32}$ Note that in our design it suffices for a 3-player clique to coordinate for activity to be optimal.

33 She argues: "when the clustering among neighbors is high, agents observe their neighbors responding to similar local conditions and play as if they were playing in a small groups instead of on a much larger network”.
} 
period, (the same group of 20) people are randomly allocated to the nodes of the network, are informed of their degree, and then choose to be active or inactive. The network remained the same for all 40 periods. We had three treatments, one for each of the networks that, as explained below, differ in terms of connectivity and clustering. The experimental instructions (to the treatment corresponding to Network 1) are provided in Online Supplement C.

Table 3 gives a summary of network characteristics for these networks. Note that networks 2 and 3 are formed by adding seven links to network 1, that both networks 1 and 2 have zero clustering whereas there is positive clustering in network 3, and that networks 2 and 3 have the same level of connectivity and the same degree distribution. Hence both networks 2 and 3 are more connected than network 1, but network 3 additionally has a higher degree of clustering.

[Table 3]

The equilibrium predictions (in pure strategies) are shown Table 4 (see the Appendix for a derivation of these results). As we can see, with this design, all three networks have the same set of (pure-strategy) equilibria: 1) An efficient equilibrium, in which players with degree higher than 1 are active, and 2) a secure (inefficient) equilibrium, in which all players are inactive.

\section{[Table 4]}

A comparison of networks 1 and 2 allows us to study the robustness of the connectivity effect on the selection of the efficient equilibrium in the large-network case, and the comparison of networks 2 and 3 allows us to study the effect of clustering. As in Experiment 2, our (behavioral) hypotheses are that connectivity and clustering enhance the selection of the efficient equilibrium, and that subjects with higher degrees are active with a higher frequency.

For each of the three treatments (different 20-person networks), we conducted three sessions, each with 20 new participants, yielding a total of 180 new participants, at the University of Innsbruck in December 2012 and January 2013.

\section{Results}

\subsection{Experiment 1}

\section{Measurement}

We analyze our data with an econometric model. We estimate the probability of being active as a logistic function of explanatory variables listed in the following paragraph. We have 
arranged the data as a panel where the unit of observation is a participant who is observed for 40 periods. The models are estimated using random effects and are shown in Online Supplement B.

The explanatory variables in the econometric model of the complete-information data are period, dummies for player position, all interactions between period and these dummies, and the measured level of risk aversion. One model is estimated using data from sessions with substitutes and another model is estimated using data from sessions with complements. The results are summarized by the estimated probabilities of being active computed by player position, network, and treatment (see Table 5 below and Online Supplement B). The explanatory variables in the econometric model of the incomplete-information data are period, a dummy for the connectivity (with $p=0.2$ as benchmark), and dummies for a player's degree, interactions across these variables, and the measured level of risk aversion. The results of this model are summarized by the marginal probabilities computed with respect to connectivity and degree (see Table 6 below).

\section{Complete information}

Table 5 presents the summary statistics for behavior in the three networks for both substitutes and complements with complete information, as well as the estimated rate of activity. Figure 2 shows the evolution per network and position across the 40 periods. $^{34}$

[Table 5 and Figure 2]

\section{Strategic Substitutes}

The main observation is that the equilibrium where $\mathrm{A}, \mathrm{C}$ and $\mathrm{E}$ are active, and $\mathrm{B}$ and $\mathrm{D}$ inactive (denoted ACE/BD henceforth) is focal in all networks. There is strong support for this. ${ }^{35}$ Averaging the absolute difference between the theoretical prediction and the observed behavior over all nodes, individual play is consistent with the equilibrium ACE/BD in 87.6 percent of all cases, which is (one of) the most secure equilibrium. There is no support for any of the other equilibria, ${ }^{36}$ so the problem of equilibrium multiplicity does not seem to be present behaviorally. In 52.5 percent of the observations the groups fully coordinate on this equilibrium; this is

\footnotetext{
${ }^{34}$ There are eight subjects in each network position in this treatment. Thus, the maximum number of observations behind each circle in Figure 2 is eight.

${ }^{35}$ The weakest support is from player $\mathrm{C}$ in networks where he or she has degree 2. Even so, there is a strong trend over time towards $\mathrm{C}$ being active, as this rate increases from 58.7 percent in the first 20 periods to 76.6 percent in the final 20 periods with the Orange network and from 52.8 percent to 80.8 percent with the Green network. Similarly, player C's activity rate increases from 90.2 percent to 98.4 percent with the Purple network.

${ }^{36}$ While there is scope for considerations of learning across games (see Mengel, 2012, for a related argument in the context of two-player games) that, for instance, might favor play of the equilibria that are common across the different games (i.e., networks in our case), since we have two equilibria (one efficient and one more secure) that are common to the three networks, this would not help in predicting which equilibrium would actually be selected.
} 
increasing over time, 36.9 (68.1) percent in the first (last) 20 periods. In fact, the correlation coefficient between the period and the average frequency of equilibrium play is $0.724(p<0.01)$.

The econometric analysis confirms our previous impressions. In all networks with strategic substitutes, the estimated activity probability for positions $\mathrm{A}$ and $\mathrm{E}$ is close to 100 percent. On the other hand, the estimated activity probability for positions B and D is never more than 10 percent. While position $\mathrm{C}$ has a lower estimated activity rate than positions $\mathrm{A}$ in $\mathrm{E}$ in networks Orange and Green, being active is still by far the most likely outcome for position C. Thus, the equilibrium ACE/BD prevails in all networks. In Figure B.1 (in Online Supplement A), we see that this regularity is present in every group.

Note that, across all possible equilibria, ACE/BD is the one that involves a maximum number of active players; i.e. it is not fully efficient, since three players pay the cost instead of two, with complete coverage in both cases (the net social benefit is 350, compared to the social benefit of 400 with only two active players). However, it can be argued that the selected equilibrium $\mathrm{ACE} / \mathrm{BD}$ is more stable than the equilibria where only two players are active. To see this, consider any of the three networks and the equilibrium ACE/BD. If any player who is active deviates to inactivity, only the deviating player incurs a loss (of 50) and, from that configuration, only such a player would have incentives to switch his action (to become active again), leading back to the initial ACE/BD equilibrium. ${ }^{37}$ On the other hand, after a deviation of a player to inactivity in the efficient equilibrium, at least two players would have incentives to become active, one of them being the deviator. ${ }^{38}$ Boncinelli and Pin (2012) provide support for this explanation, as they show that in Best Shot Games played in networks under complete information, the equilibria that involve a maximum number of active players are the only stochastically-stable ones under a class of evolutionary dynamics. ${ }^{39}$ This result applies directly to our set-up. ${ }^{40}$ Summarizing, there is a trade-off between efficiency and stability.

\footnotetext{
${ }^{37}$ Note that there is one exception. In the purple network, if player E becomes inactive, both players E and D have incentives to switch their action. However, note that no matter who switches, we would end up in one of the most secure equilibrium (the same argument applies to the equilibrium ACD/BE).

${ }^{38}$ For example, in the equilibrium BE/ACD in the Orange network: if B deviates, A, B and C all incur a loss of 50.

${ }^{39}$ The stochastically-stable states are those robust enough to be observed a significant fraction of time under a slightly perturbed dynamic (in the limit when the mutation probability goes to zero). Specifically, Boncinelli and Pin (2012) find that our more secure equilibria are the only stochastically-stable ones under (perturbed) myopic best response dynamics such that the rate at which active players mutate (make mistakes) is of an order sufficiently larger than that of inactive players. Otherwise, they show that all the equilibria are stochastically stable.

${ }^{40}$ The equilibrium ACE/BD is the only stable one in the Orange and Green networks, with an additional stable (but inefficient) equilibrium of ACD/BE in the Purple network. However, as shown in Online Supplement D, with the
} 
Result 1: With complete information and strategic substitutes in Experiment 1, agents' behavior in all three networks is consistent with the inefficient, but stochastically-stable equilibrium ACE/BD. Coordination on this equilibrium increases over time.

\section{Strategic Complements}

For complements, we see an impressive rate of play (96.1 percent) consistent with the unique equilibrium (no activity) in the Green and Purple networks. ${ }^{41}$ The Orange network admits two equilibria, with either three active players or none. Here the play resembles the active equilibrium, as players $\mathrm{B}, \mathrm{C}$, and $\mathrm{D}$ are active 74.0 percent of the time, and players $\mathrm{A}$ and $\mathrm{E}$ are inactive 95.6 percent of the time. This is also the efficient equilibrium, since players B, C, and D each earn more than with the inactive (secure) equilibrium. Thus, we find strong support for the theoretical predictions (players mostly play an equilibrium), and with multiplicity there is successful coordination by players (in a clique) on the efficient equilibrium. At the group level (see Figure B2 in Online Supplement A), three of the four matching groups coordinate quite well on this equilibrium. Over time, equilibrium play becomes more frequent, with a correlation coefficient between the period and the frequency of equilibrium play of $0.622(p<0.01)$.

The estimated probabilities of being active confirm this impression (Table 5). In all networks, this estimate for positions $\mathrm{A}$ and $\mathrm{E}$ choosing active is close to 0 . This is also true for positions B, C, and D in the Green and Purple networks, while in the Orange network B, C and $\mathrm{D}$ are predominantly active (the estimated activity rates are respectively $0.843,0.746$ and 0.813 ).

Result 2: With complete information and strategic complements in Experiment 1, players in the Green and Purple networks play the unique equilibrium, while players in the Orange network behave largely consistently with the efficient equilibrium BCD/AE. There is increasing coordination on the equilibrium over time.

Note the difference in outcomes between strategic substitutes and complements: while people select the inefficient equilibrium (the most secure one) with substitutes, with complements they select the efficient one. We can explain this difference by looking at the relation between efficiency and private incentives. With complements, the two equilibria are Pareto-ranked, and even if the inefficient equilibrium is secure, with complete information participants are able to successfully coordinate on the efficient equilibrium, which is (weakly)

Purple network ACE/BD is (generalized) risk-dominant while ACD/BE is not. In any case, one could conjecture that an additional explanation for the fact that (in the case of the Purple network) the ACE/BD equilibrium is mostly played, whereas there is no support for the ACD/BE equilibrium, could be an aesthetic preference for symmetric outcomes (since nodes ACE are symmetrically spatially located in the network as depicted in Figure 1).

${ }^{41}$ Of course, A and $\mathrm{E}$ will never wish to be active, since the maximum possible gain is less than the cost. 
beneficial for all of them. With substitutes there are more subtle considerations, since the equilibria are not Pareto-ranked and, therefore, there are individuals in different positions that (strictly) prefer different equilibria. Our results suggest that, in this case, security/stability considerations play an important role in the equilibrium selected (in line with the findings of Rosenkranz and Weitzel, 2012).

\section{Incomplete information}

Table 6 presents the summary statistics for behavior with incomplete information and both strategic substitutes and complements under each probability regime, as well as the marginal effects on activity. Figure 3 shows the evolution per network and position across the 40 periods for both substitutes and complements.

[Table 6 and Figure 3]

\section{Strategic substitutes}

For strategic substitutes we observe that, in each case ( $p=0.2$ and $p=0.8$ ), modal play coincides with play in the unique equilibrium. ${ }^{42}$ The correspondence is excellent for degrees 1 and 3, but less so for degree 2. Overall, 87.6 percent of all choices were consistent with equilibrium play when $p=0.2$ and 84.0 percent when $p=0.8$.

Regarding the effect of connectivity within a particular degree (recall that higher values of $p$ imply higher connectivity), Table 6 shows no significant difference in the behavior of players with degree 1 across the values of $p$. For players with degree 2, the probability of being active is significantly higher when $p=0.8$, with a marginal effect of 0.547 ; for players with degree 3 , this probability is marginally-significantly higher with $p=0.8$, although the marginal effect is small (0.024). Overall, our data are consistent with the equilibrium prediction.

Next consider the effect of degree on behavior. Participants with degree 2 are much less likely to choose activity than those with degree 1 ; the decrease is quite large when $p=0.2$ and much smaller when $p=0.8$. People with degree 3 have a much lower probability of choosing activity than do people with degree 1 , for both values of $p$. Comparing degree 3 to degree 2 , the probability of choosing active is significantly lower for participants of degree 3 , with a large

\footnotetext{
42 The proportions are 94.8, 71.8 and 98.9 percent of the time, respectively, for degree 1,2 and 3 when $p=0.2$, and 92.9, 59.5 and 89.9 percent when $p=0.8$.
} 
difference when $p=0.8$ and a much smaller one when $p=0.2 .{ }^{43}$ All of the differences across the probability values are in the direction of the theoretical prediction. ${ }^{44}$ Hence, our analysis suggests that the expected effects of connectivity and degree are observed in the lab.

We can also examine behavior over the course of the 40 periods. Behavior is quite stable for players with degrees 1 and 3 (and very close to the equilibrium prediction). The frequency of choosing to be active for players with degree 2 is always below $1 / 2$ when $p=0.2$, and mostly above $1 / 2$ when $p=0.8$; this qualitatively follows the equilibrium prediction, although deviations are observed. We note that when $p=0.8$, players of degree 2 display a convergence to the equilibrium. Overall, the correlation coefficient between the period and the frequency of equilibrium play is 0.233 (not significant) with $p=0.2$ and is 0.594 ( $p$-value $<0.01$ ) with $p=0.8$.

Summarizing, when players face a game of strategic substitutes with low connectivity, individual play and the level of coordination is stable over time. With higher connectivity there is a strong trend to the unique equilibrium and an increasing level of coordination.

Result 3: Under incomplete information and strategic substitutes in Experiment 1, people consistently play the unique equilibrium and the probability of activity is decreasing with the degree and increasing with connectivity.

\section{Strategic complements}

Now consider the case of strategic complements. When $p=0.2$, there is a unique equilibrium (all inactive), and play by people with degrees 1 and 2 is strongly consistent with the equilibrium prediction (98.0 and 82.1 percent). However, subjects with degree 3 are inactive only a bit more than half the time (55.6 percent). Still, in the aggregate, individual play is consistent with the equilibrium prediction six out of seven times. When $p=0.8$, there are two pure-strategy equilibria. In the secure one, no players are active, but in the efficient one players with degree 2 and 3 are active. While the behavior of individuals with degree 1 is strongly

\footnotetext{
${ }^{43}$ The marginal effects for degree 2 versus degree 1 are -0.816 and -0.273 for $p=0.2$ and $p=0.8$, respectively; for degree 3 versus degree 1 , these are -0.980 and -0.961 for $p=0.2$ and $p=0.8$, respectively. Finally, the marginal effects for degree 3 versus degree 2 are -0.687 and -0.164 for $p=0.2$ and $p=0.8$, respectively.

${ }^{44}$ The fact that players with degree 2 play equilibrium strategies less frequently than players with degrees 1 and 3 may reflect their lower cost from deviating: (I) Consider the case $p=0.2$, where players with both degree 2 and degree 3 are inactive in equilibrium. A player with degree 3 has more chances of being linked with an active player than does a player with degree 2 (i.e. the cost of deviation for a player with degree 2 is lower); (II) Consider the case $p=0.8$. Here players with both degree 2 and degree 1 are active in equilibrium. Similarly, in this case, the cost of deviating to become inactive is lower for players with degree 2 than for players with degree 1 (a deviating player with degree 2 is more likely to be linked to an active player), and we could expect more deviations from them.
} 
consistent with these equilibria (98.2 percent), the evidence on the behavior of individuals with degrees 2 and 3 is mixed, with activity rates of 31.0 and 51.0 percent respectively.

Regarding the effect of connectivity within a particular degree, the behavior of players with degree 1 does not significantly differ across the values of $p$. Players with degrees 2 and 3 are significantly more likely to choose to be active for the higher values of $p$, reflecting attempts by the players of higher degree to coordinate on activity. However, these attempts are largely unsuccessful over time. The decline over the course of the session is faster when $p=0.2$ (where activity is not present in any equilibrium) and slower when $p=0.8$.

Concerning the effect of the degree, a person with degree 2 is significantly more likely to be active than a person of degree 1 , but this difference is considerably larger with $p=0.8$ than with $p=0.2$ (the marginal effects are 0.153 and 0.041 , respectively). This qualitatively supports the threshold equilibria identified by Galeotti et alii (2010) that, as explained above, depend on connectivity. Note that the inefficient (inactive) equilibrium is still present in the scenario with $p$ $=0.8$, so this result is in line with our behavioral hypothesis on the effects of connectivity on equilibrium selection (since players with degree 2 are active in the efficient equilibrium when $p$ $=0.8$ ). The same relationship holds between players with degrees 1 and 3 , with higher marginal effects when $p=0.8$ (0.562 versus 0.328 with $p=0.2$ ). Finally, players of degree 3 are significantly more likely to be active than players of degree 2 , for all values of $p$. This evidence, not predicted by theory, is in line with our behavioral hypothesis on degree, due to the greater incentive for players of degree 3 to coordinate on the efficient equilibrium.

The pattern is revealing. It seems that subjects with higher degrees (particularly with degree 3) attempt to coordinate on profitably being active. But these attempts at efficiency diminish over time, with low or very low rates of activity for everyone by the end of the session; the correlation coefficient between the period and the average frequency of equilibrium play is 0.926 for $p=0.2$, and 0.639 for $p=0.8$, significant at the one percent level. So it seems that the inefficient (but secure) equilibrium would prevail in the long run. Our interpretation is that coordination problems lead participants to eventually play this generalized risk-dominant equilibrium. In any event, modal play (in the aggregate) corresponds to this no-activity case.

Summarizing, when players face a game of strategic complements, individual play with low connectivity converges to the unique equilibrium with an increasing level of coordination; individual play with higher connectivity appears to converge to the inefficient equilibrium. 
Result 4: Under incomplete information and strategic complements in Experiment 1, the modal play coincides with the unique equilibrium with lower connectivity, while the probability of activity increases with the degree and connectivity. With high connectivity, there is convergence towards the inefficient (but secure) equilibrium.

\section{Certainty versus uncertainty with strategic complements}

We find a difference in play when it is certain that the Orange network is in force (complete information) and when this is only very likely $(p=0.8){ }^{45}$ The question that arises is whether this difference is driven by there simply being any element of uncertainty regarding the network in force. Recall that the result with complete information is driven by the ability of the BCD clique (i.e., the positions with degree 2 or 3) to coordinate on activity, and that the potential benefit of such coordination is only one-third of the potential loss from trying. Perhaps even a tiny amount of uncertainty will make such coordination too difficult to achieve.

Accordingly, we conducted another treatment in which the probability that the Orange network is in force is 0.95 . There are two equilibria: the efficient one where players with degree 2 and 3 are active (analogous to the one played when the Orange network was certain) and a second one in which all players are inactive (the inefficient one, to which behavior converged in our treatment of incomplete information with $p=0.8) .{ }^{46}$ If we observe differences between this environment and one with complete certainty, it indicates that coordination on efficient-but-risky equilibrium is too difficult without common knowledge of the precise network having been implemented. This explanation is in part based on the abundant experimental evidence that people are loss adverse and tend to overestimate small probabilities.

While the activity rate for degree- 1 players is always negligible, the aggregated activity rates observed for players with degree 2 or 3 with $p=0.95$ look considerably closer to those by players in positions C (degree 2), and B and D (degree 3) with complete information when the Orange network was in force (hereafter we denote this environment by $p=1$ ) than to those found with incomplete information and $p=0.8$. The activity rates for players of degree 2 are 31.0, 67.9,

\footnotetext{
${ }^{45}$ Note that, although we cannot strictly say that the set of equilibria under complete information with the Orange network $(p=1)$ and under incomplete information with $p=0.8$ are the same (since the strategy spaces differ), they are indeed equilibria of the very same nature, and the comparison is meaningful. Clearly, the inefficient equilibrium (all inactive) reflects the very same arguments in both cases. In the efficient equilibrium with $p=1$ positions $\mathrm{B}, \mathrm{C}$ and $\mathrm{D}$ (all degree 2 or 3 ) are active, and positions $\mathrm{A}$ and $\mathrm{E}$ (degree 1) are inactive and, in the efficient equilibrium with $p=0.8$, players with degree 2 and 3 are active, while inactive with degree 1 (since with high probability they are in the BCD clique of the Orange network, as in the Green and Purple networks the incentives are to be inactive). ${ }^{46}$ When $p=0.95$, if players are coordinated in the efficient equilibrium, the probability of a loss when an agent has degree 3 is approximately $1 \%$, while this probability is $7 \%$ when he has degree 2 . Then, assuming coordination in the efficient equilibrium, over a total of 40 periods an individual would experience, on average, one period of losses.
} 
and 72.8 percent for $p=0.8,0.95$, and 1 , respectively, while the corresponding activity rates for players of degree 3 are 51.0, 75.7, and 74.6 percent. So at first glance it seems that there is no pure uncertainty effect. However, the patterns of play over time suggest otherwise.

[Figure 4]

Simple inspection of Figure 4 shows a clear negative trend in the rate of activity in both treatments of incomplete information ( $p=0.8$ and $p=0.95$ ), but no evidence of decay with complete information $(p=1)$. Table 7 provides analytic evidence of this visual evidence:

[Table 7]

We report the estimated rates of activity by treatment and degree for the average period (20) and for the last period (40). ${ }^{47}$ Comparing each of these rates in the two treatments with incomplete information, we find a clear and highly significant evidence of the connectivity effect for degrees 2 and 3. Across the treatments $p=0.95$ and $p=1$ we find that, while in period 20 there is no significant difference, in period 40 the differences are significant at $5 \%$ in the direction of a higher activity rate with complete information. Table 7 also shows that the trend in the activity rate for degree 2 is significantly more negative with $p=0.95$ than with complete information. So it seems that the effect of uncertainty per se changes over time, indicating that learning plays a role in how participants react to uncertainty. The trends suggest that the inefficient equilibrium will eventually prevail with incomplete information, while the efficient equilibrium prevails with complete information. Thus, it appears that uncertainty per se (regardless of the degree) is enough to derail attempts to coordinate on the efficient equilibrium.

One may wonder why the effect of uncertainty does not appear until time has passed in the sessions. We suspect that this is a contagion effect in the coordination game. When players observe that some other players are inactive (together with the fact that they also face an uncertain context), they also become inactive. So we feel that the uncertainty matters together with the coordination context players face: Given the uncertainty, players may have different thresholds regarding how much perceived inactivity induces them to become inactive.

Result 5: We see that even a very small amount of uncertainty about the network in force $(p=0.95)$ can lead to considerable differences over time with respect to behavior with certainty, derailing attempts to achieve the efficient equilibrium.

\footnotetext{
${ }^{47}$ We estimated the probability of being active using a logit panel model with random effects.
} 


\subsection{Experiment 2}

As mentioned earlier, we only consider strategic complements in Experiment 2, as these are better suited for testing equilibrium selection.

\section{Complete information}

Table 8 presents summary statistics for behavior in the three networks with complete information, as well as the marginal effects on activity. In all networks, the modal play corresponds (to varying degrees) to the efficient equilibria. For the Blue network, all positions except A should be active. Indeed A is never active, and the rates for B, C, and E (with three neighbors) are well over 90 percent. The activity rate for position $\mathrm{D}$, with exactly two neighbors, is only 76 percent. $^{48}$ In the Red network, position $\mathrm{A}$ is never active, so $\mathrm{B}$ has only one potentially-active neighbor and so is rarely active. C has three links and is nearly always active. However, positions D and E are only active 75-80 percent of the time; this pattern is similar to that for the Blue network. Note the difference in play for B versus D and E, even though each has two neighbors. Finally, the efficient equilibrium is least likely for the Brown network. While the play of positions A and B (one and three links, respectively) conforms well to the equilibrium predictions, C, D, and E (two links) have activity rates between 58 to 67 percent. $^{49}$

There is a positive effect of degree, since within each network the frequency of activity is higher for players with degree 3. We also see a positive effect of clustering, as both players with degree 2 who should be active in the efficient equilibrium and players with degree 3 are more likely to be active in the Red network (with clustering coefficient of 0.5 than in the Brown network (clustering coefficient 0 ). There are also higher activity rates for the more connected (and more clustered) Blue network (clustering coefficient 0.6) than the others. So the results are in line with our behavioral hypothesis. Figure 5 shows the evolution for each network and position over time. We do not find any time trends for any position in any of the networks.

\section{[Table 8 and Figure 5]}

For the econometric analysis we estimate a model analogous to that described in Experiment 1. Across networks and positions (at the average period and average risk levels) we see that players predicted to be active in the efficient equilibrium for each network have an

\footnotetext{
${ }^{48}$ Recall that activity with complements is only profitable with at least two active neighbors.

${ }^{49}$ It is a bit puzzling that B is more active than C, D, and E, since B's third neighbor is certainly inactive and so B, $\mathrm{C}, \mathrm{D}$, and $\mathrm{E}$ are essentially equivalent; it seems that B is somehow affected by the presence of the third link.
} 
estimated activity rate ranging from 0.581 to 0.996 . These results confirm that the efficient equilibrium prevails for all three networks. Note that the only difference between the Blue and Red networks is one extra link in the Blue network; this extra link changes the probability that B players choose to be active from 92.86 percent to 18.52 percent, a highly dramatic decrease that is consistent with the equilibrium prediction (in the efficient equilibrium, position B is active in the Blue network, but inactive in the Red one).

Result 6: In Experiment 2 with complete information, the efficient equilibrium prevails in every network. There is a strong effect of degree on activity, as well as an effect of clustering.

\section{Incomplete information}

Table 9 presents the summary statistics for behavior with incomplete information, as well as the marginal effects on activity. Figure 6 shows the evolution per degree over time.

[Table 9 and Figure 6]

Modal play for all degrees corresponds to the efficient equilibrium both when $p=0.2$ and $p=0.8$. In both treatments there is a positive effect of degree on activity levels (degree 3 is, respectively, 22.4 and 17.1 percentage points more active than degree 2). Regarding the effect of connectivity, there is no difference across values of $p$ for players with degree 3 , but there is a small difference for players with degree 2 (6.7 percentage points) that suggests a positive effect on the selection of the efficient equilibrium. ${ }^{50}$ The main difference in time trends across the two values of $p$ is that there is a clear negative trend for players with degree 2 when $p=0.2$, while the trend with $p=0.8$ is constant (or even slightly positive). There are no time trends for players of degree 1 (who are almost never active) or for players of degree 3 (who are almost always active).

To test the significance of the effects for degree and connectivity, we report (in the bottom part of Table 9) the marginal effects for players of each degree, estimated using an econometric model similar to that used for Experiment 1. We see a significant degree effect on the likelihood of activity. Comparing rates for degree 1 and the other degrees gives very large marginal effects. There is also a small but significant effect of degree for players of degrees 2 and 3; this is not predicted in equilibrium but is in the direction of our behavioral hypothesis. The estimated increase in the probability of activity is 5.2 and 9.3 percentage points when $p=0.2$ and $p=0.8$, respectively. Both marginal effects are significant at the $10 \%$ level with two-tailed tests.

\footnotetext{
${ }^{50}$ While a 6.7 percentage-point increase is certainly not large, it is nevertheless nearly one-quarter of the maximum 27.5 percentage-point increase possible from the activity rate with $p=0.2$.
} 
Finally, the marginal effects of connectivity ( $p=0.8$ versus $p=0.2$ ) on the probability of activity, measured at the average risk levels, are reported in the middle of Table 9. Since Figure 6 has shown a great difference in trends across $p=0.2$ and $p=0.8$ for players with degree 2, we measure the marginal effect at both the average period (20) and the final period (40).

At period 20, there are no significant differences for any degree. This is unsurprising for degree-3 players, since the frequency of activity is already very high when $p=0.2$. However, when we use period 40 to measure the marginal effect of connectivity for players with degree 2, we find significance at the $10 \%$ level on a two-tailed test. ${ }^{51}$ Hence, there is some effect of connectivity on activity rates for players with degree 2, but people need some periods of learning before this effect kicks in. No difference is observed or predicted for players of degrees 1 or $3 .^{52}$

Summarizing, we have:

Result 7: With incomplete information in Experiment 2, modal play corresponds to the efficient equilibrium for all probability values. Once again, there is a strong effect of degree and we see evidence that the probability of activity increases with the connectivity.

\subsection{Experiment 3}

In Table 10, we display the frequencies of activity by degree and network, as well as the marginal effects on activity. As before, players with degree 1 are rarely active, with rates ranging from three to seven percent. We see a strong degree effect on the activity, as these rates increase by degree in all three networks and for each degree. In network 1, this increase is steady (three to 25 to 61 to 81 percent), while in networks 2 and 3 the rate jumps to around 90 percent for degree 2 and is almost 100 percent with degree 3 or $4 .^{53}$

[Table 10]

\footnotetext{
${ }^{51}$ The econometric analysis shows that, both for degree 2 and 3, the differences in the trends corresponding to treatments $p=0.8$ and $p=0.2$ (measured by the marginal effect of treatment on the marginal effect of period by degree) are positive and significant (at the five percent level for degree 2 and the 10 percent level for degree 3).

${ }^{52}$ Once again, the marginal effects (not reported here) of risk preference on the probability of being active are not significant for any degree or connectivity level.

${ }^{53}$ In the third session of network 3, we identified one person whose behavior was anomalous. This individual was always active when her degree was 2, whereas she was always inactive when her degree was 3 or 4 . This pattern is in stark contrast to the incentive structure of the game and the behavior of all the other 179 subjects that played either in networks 1, 2 or 3. Thus, although we keep the data of this session for our analysis, we decided to remove the data from this specific anomalous individual throughout all our analysis. Consider that without this individual, we have completely full activity for players with degree 3 and 4, while this person is never active with degree 3 or 4 (but is active at a lower degree); furthermore, network 2 has full activity for players of degrees 3 and 4 . Thus, if we include this individual and compare behavior in networks 2 and 3, we would find an odd 'clustering' effect.
} 
We find a clear connectivity effect when behavior is compared across network 1 and either network 2 or 3, as the activity rates in networks 2 and 3 (higher connectivity than network 1) are much higher than in network 1 for players with multiple connections. In Figure 7 we observe the evolution of activity in each network (by degree). The results show convergence to the inefficient equilibrium in network 1 and clear adherence to the efficient equilibrium in networks 2 and 3 . This shows a clear and strong connectivity effect.

[Figure 7]

Regarding clustering, we can compare network 2 to network 3 (with a clustering coefficient of zero in network 2 and a coefficient of 0.31 for network 3). Since players with degree 3 and 4 are already fully active in network 2, to identify a clustering effect we can only consider players with degree 2. Still, the activity rate in network 2 is already very high (88.1 percent) for degree 2 and the probability of being active is only slightly higher in network 3 (92.9 percent). Nevertheless, the modest 4.8 percentage-point increase does represent more than 40 percent of the maximum 11.9 percentage-point increase possible. We do see some suggestive evidence of a clustering effect at the session level: In all three sessions of network 2, there is only partial activity for degree-2 players. By comparison, players with degree 2 in network 3 are fully active in two of three sessions, with partial inactivity for degree 2 players in network 2.

In order to study the significance of these effects, as before we estimate a logit panel-data model with random effects and report the marginal effects across networks in relation to the probability of being active (measured at the average period and risk levels). The estimations show a significant connectivity effect on the choice of being active (the marginal effect for players with degrees 2, 3 and 4, comparing network 1 to either of networks 2 or 3). However, the clustering effect (marginal effect of network 3 over network 2, measured for players with degree 2 ) is not significant, perhaps due to the high activity rate in network 2 (ceiling effect).

Summarizing, we have:

Result 8: Activity rates increase with players' degree in all three networks in Experiment 3. Comparing across networks, we find a clear and strong effect of connectivity, as well as suggestive evidence of a clustering effect.

\section{Discussion}

In this section, we address issues of behavior with respect to equilibrium predictions. We first consider how well the experimental data fit the theoretical predictions, and then discuss the 
extent to which potential multiple equilibria manifest in each treatment, as well as the issue of convergence over time. Finally, and perhaps most importantly, we examine the underpinnings of factors that appear to drive the selection of a particular equilibrium or equilibria.

\subsection{Conformance of the experimental results to theory and hypotheses}

\section{Experiment 1}

The results in Experiment 1 are quite consistent with the theoretical predictions for behavior in our games. These results not only provide very strong qualitative support, but also surprisingly strong quantitative support. With complete information and strategic substitutes, 87.6 percent of choices correspond to a (stochastically-stable) equilibrium. When the game involves strategic complements, play corresponds to the predicted equilibrium 96.1 percent (100 percent in the last 10 periods) of the time when it is unique. Matters are a bit more complicated when there are two equilibria. While overall the efficient equilibrium is played 74 percent of the time by the players who should be active (82.6 percent over all players), one of the four 10person groups converged to the no-activity equilibrium. In the last 10 periods of the session, the activity rate for players B, C, and D combined was 83.9 percent overall for three of the groups, but was only 11.1 percent for the other group. ${ }^{54}$ So we see heterogeneity across groups.

The results with incomplete information are particularly striking, given the much greater complexity of this environment. With substitutes, play is consistent with the unique equilibrium 87.6 percent of the time when $p=0.2$ and 84.0 percent of the time when $p=0.8$. Play in the last 10 periods is even more consistent with the equilibrium for both $p=0.2$ (91.0 percent) and $p=$ 0.8 (91.2 percent) ${ }^{55}$ But these percentages are relatively low for subjects with degree 2 , as there is a substantially lower expected cost if one deviates from equilibrium play.

With incomplete information and complements, play is consistent with the unique equilibrium 85.7 percent of the time (97.2 percent in the last 10 periods) when $p=0.2$. When $p=$ 0.8 , there is an additional equilibrium in which players of degree 2 and 3 are active. The overall activity rates are 31.0 percent for players with degree 2 and 51.0 percent for players of degree 3 ,

\footnotetext{
${ }^{54}$ Purely in terms of expected value, being active pays off for player B, C, or D if the chance that both of the other two players are also active is at least $2 / 3$, which corresponds to 81.6 percent for each player without correlation. But a taste for social efficiency (Charness and Rabin, 2002) may lower this threshold.

${ }^{55}$ The rates in the last 10 periods for degrees 1 , 2, and 3 with $p=0.2$ are 99.5 percent, 72.7 percent, and 100 percent, respectively. The rates in the last 10 periods for degrees 1 , 2, and 3 with $p=0.8$ are 94.6 percent, 78.4 percent, and 95.2 percent, respectively.
} 
painting a murky picture. However, these rates decline to 4.3 percent and 14.9 percent in the last 10 periods, so that behavior appears to be converging to the no-activity equilibrium.

The observed effects of degree and connectivity on activity support the notion of threshold equilibria, which implies a negative relationship between degree and activity with substitutes, but a positive one with complements. Furthermore, activity rates for agents with degrees 2 or 3 are higher for both complements and substitutes with higher connectivity (agents with degree 1 should never be active with complements for either $p$-value, but should always be active with substitutes for either $p$-value). ${ }^{56}$

\section{Experiment 2}

The results of Experiment 2 (with strategic complements only) are more mixed, but provide strong qualitative and some quantitative support for equilibrium play. In this design, there is always a secure equilibrium with no activity and an efficient equilibrium (not the same across networks) with activity by a proper subset of the players. With complete information, 81.2 percent of the overall choices correspond to the efficient equilibrium. The likelihood is highest for the Blue network, lower for the Red network, and lowest for the Brown network. Note that this pattern matches the number of 3-player cliques in each network, showing the difficulties in successful coordination on activity. All four groups coordinate on the efficient equilibrium with the Blue network, but one group in the Red network and two groups in the Brown network fail to do so. ${ }^{57}$

The equilibria with incomplete information are the same for $p=0.2$ and $p=0.8$, with a no-activity equilibrium and one in which players with degrees 2 and 3 are active; players of degree 1 should never be active. ${ }^{58}$ While activity rates are slightly higher for degree-2 players with more connectivity (79.21 percent versus 72.48 percent), the activity rates for degree-3

\footnotetext{
${ }^{56}$ Summarizing, the activity rates with substitutes for degrees 1,2 , and 3, respectively, drop from 95 to 28 to 1 percent with $p=0.2$ and from 93 to 60 to 10 percent for $p=0.8$. The activity rates with complements increase from two to 18 to 44 percent for $p=0.2$ and from two to 31 to 51 percent for $p=0.8$. Regarding connectivity, the comparisons with substitutes across $p=0.2$ and $p=0.8$ are 28 versus 60 percent for degree 2, and one versus 10 percent for degree 3; the respective comparisons with complements are 18 versus 31 percent (44 versus 51) for degree 2 (3).

${ }^{57}$ The rate for players predicted to be active in the Red network is 83.5 percent for the three coordinating groups, but only 42.5 percent for the other group; the corresponding rates for the final 10 periods are 79.1 and 22.2 percent. By the same token, the rate is 86.1 percent for the two coordinating groups in the Brown network and is 38.2 percent for the two non-coordinating groups; the corresponding rates for the final 10 periods are 88.3 and 18.7 percent.

${ }^{58}$ Recall that there was also an additional evanescent (weak) equilibrium for $p=0.8$, in which a player with degree 2 is not active. Yet, this equilibrium does not have any behavioral impact (cf. Footnote 31).
} 
players are essentially the same with low and high connectivity (94.87 and 96.36 respectively). There is no time trend except for degree-2 players and low connectivity, as the respective activity rates in the last 10 periods for degree-2 players and degree-3 players are 62.14 and 93.86 percent when $p=0.2$ and 78.95 and 98.06 percent when $p=0.8$. Indeed with $p=0.2$, while two groups fully converge to the efficient equilibrium (99.38 percent activity in the last 10 periods for players with more than one degree), the other two do not (47.50 percent activity in the last 10 periods) and in fact seem to be headed toward the no-activity equilibrium.

The effects of degree are consistent with our hypothesis, as there is more activity with higher degree with both complete and incomplete information. We also confirm the effect of connectivity in the latter case. With complete information, the increased connectivity and clustering in the Blue network lead to higher activity rates than in the other networks. In addition, comparing behavior in the Red network to that in the Brown network allows us to identify a pure effect of clustering, holding connectivity constant. Table 8 shows that, for the players predicted to be active in the efficient equilibrium, the frequencies of activity in the Red network is higher than in the Brown network, with aggregate frequencies of 71.0 percent and 64.2 percent, respectively. Since the frequency of activity is higher in the Red network than in the Brown network for all four groups, a binomial test that conservatively considers each group to be only one independent observation gives $p=0.062$ on a (justified) one-tailed test. So there does appear to be an effect from clustering.

\section{Experiment 3}

In this difficult stress test with 20-person networks, the theoretical predictions do fairly well. The activity rates increase steadily by degree, at least up to the point where the activity rate is near 100 percent (for degrees 3 and 4 in networks 2 and 3). All three networks had the same two equilibria, one with no activity and an efficient one with activity by all players with multiple links. We have hypotheses regarding connectivity and clustering that can be tested by comparing activity rates across network 1 and network 2 (change in connectivity only) and across network 2 and network 3 (change in clustering only), respectively. Figure 7 indicates that the efficient equilibrium predominates in networks 2 and 3. However, there is considerable decay over time with network 1 for players with multiple links.

In fact, the pattern becomes clearer if we examine the session-level data for network 1 . In one session, activity rates were completely stable, about five percent, 43 percent, 85 percent, and 
100 percent for degrees $1,2,3$, and 4, respectively, both on average and for the last 10 periods. There is sharp decay in activity rates in both of the other sessions, where the average activity rates in the last 10 periods are zero, 2.5, 17.5, and 62.5 percent for degrees $1-4$, respectively. While some hubs still cling to the possibility of gaining through activity, we speculate that they would eventually give up and the no-activity equilibrium would be reached.

So adding seven links to the 20 in network 1 greatly affects behavior. Activity rates are lower in all sessions of network 1 than in any sessions of either network 2 or network 3, and two of the three sessions with network 1 clearly converge to the no-entry equilibrium. Yet, for some reason, the efficient equilibrium is played in networks 2 and 3 . This brings us to our next section.

\subsection{Equilibrium selection}

We find some strong and interesting patterns, and generally a strong adherence both qualitatively and quantitatively to the theoretical predictions. Nevertheless, a key issue for policy is that of equilibrium selection, where theory is typically silent. How can we predict which of multiple equilibria will prevail? Our results shed some light on this issue and perhaps permit us to make some conjectures about equilibrium selection in games on networks.

Overall, there is a strong tendency for a group to converge to one of the theoretical equilibra. ${ }^{59}$ When there is a unique equilibrium, this is played almost universally. We again mention that this is also the case in games of incomplete information, where players don't even know the network that has been drawn, let alone their position in it.

But there are definite patterns in the data that beg for an explanation. In Experiment 1, a particular equilibrium is played in all three networks with strategic substitutes and complete information. This equilibrium, with three active players, is secure and nearly as efficient as the equilibrium in which only two players are active. ${ }^{60}$ With strategic complements, only the Orange network has an equilibrium involving activity; this requires full coordination by the members of a 3-person clique, as the potential loss from being active is three times the potential gain. This clique is successful in coordinating on the efficient equilibrium in three of the four groups, with an overall activity rate of 86.08 percent in the final 10 periods (11.11 percent activity rate in the other group). Yet, this kind of equilibrium does not manifest (see Figure 3) when the probability

\footnotetext{
${ }^{59}$ Throughout the paper, we have ignored the existence of mixed-strategy equilibra in our networks, as these do not seem to have behavioral impact.

${ }^{60}$ Again, the net social benefit is 350 , which is 87.5 percent of the net social benefit of 400 with two active players.
} 
is 80 percent that the Orange network has been drawn. ${ }^{61}$ In Experiment 2, with complete information the efficient equilibrium is largely present in the Blue and the Red networks, which both have a clique, but less so in the clique-less Brown network. Perhaps surprisingly, the efficient equilibrium is mostly played even when there is only a 60 percent chance (incomplete information, $p=0.2$ ) that a network with (at least) one clique has been drawn (which happens in the blue and the red network). Finally, in Experiment 3, the efficient equilibrium was played in two of the networks, but not in most sessions of the less-connected third.

One general tendency we see is that people have a strong taste for coordinating on efficient outcomes. There is considerable evidence (e.g., Charness and Rabin 2002; Engelmann and Strobel 2004) that people like social efficiency. This is similar to the taste for achieving payoff-dominant outcomes (all agents receive their highest payoff), found in the experimental literature (e.g., Charness, 2000). Equilibrium selection may reflect a group's overall taste for efficiency. If many people in a group are willing to take the chance on the efficient (but risky) equilibrium with activity, they may well be able to sustain the maximum payoff stream. This is idiosyncratic across groups and will essentially fall on either side of a threshold value. Certain conditions enhance the likelihood that the efficient equilibrium is selected.

We have seen that connectivity often influences the likelihood of activity, affecting which equilibrium emerges. A more active equilibrium occurs in Experiment 1 with strategic substitutes and incomplete information when the connectivity increases; with strategic complements the more active equilibrium collapses over time. With complements and complete information, the extra link in the Orange network generally leads to successful coordination on the active equilibrium. We see some evidence of a connectivity effect in Experiment 2 with incomplete information, as half of the groups converged on the no-activity equilibrium with $p=$ 0.2 , but no groups did with $p=0.8$. Finally, there is a clear effect of connectivity when comparing behavior in networks 1 and 2 of Experiment 3 (with complements and incomplete information).

The manner in which the network is connected also matters; we consider the clustering coefficient, which reflects the number of cliques in the network. There is no direct way to test for clustering effects in Experiment 1, since the Orange network differs from the others by having

\footnotetext{
${ }^{61}$ Even if the strategy spaces differ, the equilibria in the Orange network with certainty and in the incomplete information scenario with high connectivity are of the same nature ( $c f$. Footnote 45$)$.
} 
both an extra link and a corresponding new clique. Nevertheless, the success in reaching the efficient equilibrium in the Orange network with complements and complete information suggests that clustering may have an effect. In Experiment 2, we do find a small-but-significant clustering effect with both complete and incomplete information. In Experiment 3, there is a modest increase in the activity rate for players with degree 2 when cliques are present. ${ }^{62}$

A third factor that feeds into equilibrium selection is the degree of uncertainty. To some extent, this may help to explain why cliques are more effective, since there is in some sense more certainty when a group is fully connected. And even when it is very probable $(p=0.95)$ that a clique is present, the efficient equilibrium (predominant with complements and certainty in Experiment 1) has collapsed into the no-activity equilibrium in half the groups. It is not so easy to coordinate when the loss from failure is triple the potential gain from success, and any degree of uncertainty substantially exacerbates the difficulty. With known positions, there may be a flavor of common knowledge, as each individual in the clique knows that the other individuals in the clique, etc., know the situation. Perhaps the awareness of a shared fate makes people more confident about the likelihood of successful coordination.

Thus, while people have a taste for social efficiency, the hurdle appears to be too high for some coordination problems. We have also seen that groups are willing to absorb a limited cost to attain the near-efficient (and more secure) equilibrium in Experiment 1 (positions A, C, and E active) with complete information and substitutes, rather than an equilibrium with full efficiency (one less active player). This equilibrium is stochastically-stable (see Footnote 39) under a class of evolutionary dynamics and gives seven-eighths of the total payoffs received in an efficient one. Our view is that this represents a group awareness of the riskiness of having only two active players. This is not unfamiliar in other coordination games. For example, Rosenkranz and Weitzel (2012) report that, when they find convergence, it is to equilibria where each inactive player is connected to at least two active players.

Summarizing, we see a number of intertwining factors that in combination determine the selected equilibrium in games on networks. Higher connectivity and more clustering increase activity rates and facilitate coordination on efficient outcomes. Uncertainty (incomplete information) is a negative influence on activity rates, but can be overcome when the coordination

\footnotetext{
${ }^{62}$ Players of degree 3 or 4 are fully active even without any cliques (network 2), so this can’t increase by introducing cliques (network 3 ) and in fact it remains the same.
} 
problem is less severe or when the active equilibrium is unique. There is also a strong taste for efficiency, but people will take a slight payoff reduction to ensure profits if one other person deviates from the equilibrium. No one of these factors is determinative, but can be seen as reinforcing or weakening beliefs in the likelihood of successful coordination. That said, nearly every group has largely converged to an equilibrium by the end of the 40 periods in the session.

\section{Conclusion}

Networks are a ubiquitous feature of the social and economic landscape, with important applications in the areas of bargaining, job search, political interactions, and systems compatibility, among others. The question of how network structure affects behavior is a vital one for business decisions and governmental policy. We conduct an experiment designed to test how games with strategic substitutes or complements, which are general to many economic environments, are played on a variety of networks. We include the case of incomplete information in our experimental design, and to the best of our knowledge we are one of the first to consider experimentally the challenging case of uncertainty and its effects on aspects of the network structure. In our view, there is almost always a degree of uncertainty concerning the prevailing network structure in the field, so this is a very relevant design choice.

A central issue in network theory is that of equilibrium selection, since it is more difficult to make informed policy decisions when one cannot predict the effects of network structure on outcomes. Considerable theoretical research has been conducted on trying to refine these or to gain insight into how to predict which of a multiplicity of equilibria actually prevails. Our principal objectives in conducting our experiments were to test theoretical predictions with complete and incomplete information and to provide empirical evidence that sheds light on factors that influence which equilibrium will actually prevail in practice in network settings. In fact, our results suggest that the problem of equilibrium multiplicity may in practice not be so severe. This is particularly true with complete information and substitutes in Experiment 1, where people seem to be willing to trade a relatively small difference in potential gain for an increased likelihood of actually receiving a gain. We find that a number of factors help to mediate which equilibrium prevails. There are higher rates of activity (and so higher profitability) with higher connectivity and clustering, and people have a definite taste for efficiency when there is not much uncertainty and risk is limited. 
We find that play conforms very strongly to the qualitative and quantitative theoretical predictions for whether agents are active or inactive. The degree to which this is true is impressive with complete information and, considering the cognitive challenge of making decisions under uncertainty, is somewhat startling with incomplete information. While play is remarkably close to equilibrium predictions even in early periods, this further improves in later periods, indicating that people learn over time to avoid mis-coordination. When we consider only the more 'settled' behavior in the last 10 periods, we observe strong convergence to an equilibrium for almost every group. In the case of incomplete information, we also find strong qualitative support for the predicted relationships between degree and activity and connectivity and activity. Our results are robust to a variety of smaller networks and larger networks.

Overall, we feel that experimental research such as this will be quite useful in making pragmatic choices regarding which network structure to implement and in predicting outcomes for an already-existing network structure. Given the uncertainty in the field environment, further experimental research that incorporates incomplete information and uncertainty certainly seems worthwhile. It might also be interesting to examine how communication between players in a network may have an impact on equilibrium selection. Our results have shown a remarkable degree of equilibrium play and coordination on a particular equilibrium without any communication, but it seems promising to study whether communication may even further improve successful coordination on the efficient equilibrium. Choi and Lee (2014) is a first step in this direction. Finally, it would be valuable to develop experiments involving endogenous network formation with multiple players. One major difficulty is that a large number of networks are possible, making it difficult to get enough data to draw even tentative conclusions. One approach is to provide an existing framework with some specified options, as in Charness and Jackson (2007), but that is just a start. Improved behavioral network theory may well be the result of the knowledge gleaned from this and future laboratory experiments. 


\section{References}

Ballester, C., Calvó-Armengol, A. and Zenou, Y. (2006): "Who’s Who in Networks. Wanted: The Key Player," Econometrica 74(5), 1403-1417.

Berninghaus, S.K., Ehrhart, K. and Keser, C. (2002): "Conventions and Local Interaction Structures," Games and Economic Behavior 39(2), 177-205.

Berninghaus, S.K., Ehrhart, K. and Ott, M. (2006): “A Network Experiment in Continuous Time: The Influence of Link Costs,” Experimental Economics 9(3), 237-251.

Berninghaus, S.K., Ehrhart, K., Ott, M. and Vogt, B. (2007): "Evolution of Networks - An Experimental Analysis,” Journal of Evolutionary Economics 17(3), 317-347.

Boncinelli, L. and Pin, P. (2012): "Stochastic Stability in Best Shot Network Games," Games and Economic Behavior 75(2), 538-554..

Boun My, K., Willinger, M. and Ziegelmeyer, A. (2006): "Structure d'interactions et problème de coordination: une approche expérimentale,” Revue d'Economie Industrielle 114-115, 245266.

Bramoullé, Y. and Kranton, R. (2007): "Public Goods in Networks," Journal of Economic Theory 135(1), 478-494.

Brandts, J. and Cooper, D. J. (2006): "A Change Would Do You Good .... An Experimental Study on How to Overcome Coordination Failure in Organizations.” American Economic Review 96(3), 669-693.

Burt, R. (2000): “The Network Structure of Social Capital,” in B. Staw and Sutton, R. (Ed.), Research in Organizational Behavior (Vol. 22). New York, NY, JAI Press.

Callander, S. and Plott, C.R. (2005): "Principles of Network Development and Evolution: An Experimental Study," Journal of Public Economics 89(8), 1469-1495.

Calvó-Armengol, A. (2004): “Job Contact Networks,” Journal of Economic Theory 115(1), 191206.

Calvó-Armengol, A. and Jackson, M.O. (2004): "The Effects of Social Networks on Employment and Inequality,” American Economic Review 94(3), 426-454.

Calvó-Armengol, A. and Jackson, M.O. (2007): "Networks in Labor Markets: Wage and Employment Dynamics and Inequality,” Journal of Economic Theory 132(1), 27-46.

Carpenter, J.P. (2007): "Punishing Free-riders: How Group Size Affects Mutual Monitoring and the Provision of Public Goods," Games and Economic Behavior 60(1), 31-55.

Carpenter, J., Kariv, S., and Schotter, A. (2012): "Network Architecture, Cooperation and Punishment in Public Good Experiments”, Review of Economic Design 16, 93-118.

Cassar, A. (2007): "Coordination and Cooperation in Local, Random and Small World Networks: Experimental Evidence,” Games and Economic Behavior 58(2), 209-230.

Charness, G. (2000): “Self-Serving Cheap Talk: A Test Of Aumann's Conjecture," Games and Economic Behavior 33(2), 177-194.

Charness, G., Corominas-Bosch, M. and Fréchette, G.R. (2007): "Bargaining and Network Structure: An Experiment.” Journal of Economic Theory 136(1), 28-65.

Charness, G., Feri, F., Meléndez-Jiménez, M.A. and Sutter, M. (2012) "Equilibrium Selection in Experimental Games on Networks," Departmental Working Papers, Department of Economics, University of California, Santa Barbara (02-18-2012).

Charness, G. and Gneezy, U. (2010): "Portfolio Choice and Risk Attitudes: An Experiment," Economic Inquiry 48(1), 133-146. 
Charness, G. and Jackson, M.O. (2007): "Group Play in Games and the Role of Consent in Network Formation,” Journal of Economic Theory 136(1), 417-445.

Charness, G. and Rabin, M. (2002): "Understanding Social Preferences with Simple Tests," Quarterly Journal of Economics 117(3), 817-869.

Choi, S., Gale, D., Kariv, S. and Palfrey, T. (2011): "Network Architecture, Salience and Coordination," Games and Economic Behavior 73(1), 76-90.

Choi, S. and Lee, J. (2014): “Communication, Coordination and Networks," Journal of the European Economic Association 12(1), 223-247.

Cook, K.S. and Emerson, R.M. (1978): "Power, Equity and Commitment in Exchange Networks,” American Sociological Review 43(5), 721-739.

Corbae, D. and Duffy, J. (2008): "Experiments with network formation," Games and Economic Behavior 64(1), 81-120.

Crawford, V.P. and Rochford, S.C. (1986): "Bargaining and Competition in Matching Markets," International Economic Review 27(2), 329-348.

Currarini, S., Jackson, M., Pin, P. (2009): “An Economic Model of Friendship: Homophily, Minorities, and Segregation,” Econometrica 77(4), 1003-1045.

Deck, C. and Johnson, C. (2004): "Link Bidding in a Laboratory Experiment," Review of Economic Design 8(4), 359-372.

Engelmann, D. and Strobel, M. (2004): "Inequality Aversion, Efficiency, and Maximin Preferences in Simple Distribution Experiments," American Economic Review 94(4), 857869.

Falk, A. and Heckman, J. (2009): "Lab Experiments are a Major Source of Knowledge in the Social Sciences,” Science 326(5952), 535-538.

Falk, A. and Kosfeld, M. (2003): "It's all about Connections: Evidence on Network Formation,” IZA discussion paper no. 777.

Fatas, E., Meléndez-Jiménez, M.A. and Solaz, H. (2010): “An Experimental Analysis of Team Production in Networks,” Experimental Economics 13(4), 399-411.

Fischbacher, U. (2007): "z-Tree: Zurich Toolbox for Ready-Made Economic Experiments," Experimental Economics 10(2), 171-178.

Gale, D., and Shapley, L.S. (1962): “College Admissions and the Stability of Marriage,” The American Mathematical Monthly 69(1), 9-15.

Galeotti, A., Goyal, S., Jackson, M.O., Vega-Redondo, F. and Yariv, L. (2010): "Network Games," Review of Economic Studies 77(1), 218-244.

Galeotti, A. and Vega-Redondo, F. (2011): "Complex Networks and Local Externalities: A Strategic Approach,” International Journal of Economic Theory 7(1), 77-92.

Goyal, S. and Moraga-Gonzalez, J.L. (2001): “R\&D Networks,” Rand Journal of Economics 32(4), 686-707.

Greiner, B. (2004): "The Online Recruitment System ORSEE 2.0 - A Guide for the Organization of Experiments in Economics," Working Paper Series in Economics 10, University of Cologne, Department of Economics.

Harsanyi, J.C., and Selten, R. (1988), A General Theory of Equilibrium Selection in Games, Cambridge: MIT Press.

Hendricks, K., Piccione, M. and Tan, G. (1995): "The Economics of Hubs: The Case of Monopoly," Review of Economic Studies 62(1), 83-99.

Jackson, M.O. (2008): Social and Economic Networks, Princeton University Press: NJ. 
Jackson, M.O. (2010): “An Overview of Social Networks and Economic Applications,” in the The Handbook of Social Economics, edited by J. Benhabib, A. Bisin, and M.O. Jackson, North Holland Press, 511-586.

Jackson, M.O. and Yariv, L. (2005): “Diffusion on Social Networks,” Économie Publique, 16(1), 3-16.

Katz, M. and Shapiro, C. (1994): "Systems Competition and Network Effects," Journal of Economic Perspectives 8(2), 93-115.

Kearns, M., Suri, S. and Montfort, N. (2006): “An Experimental Study of the Coloring Problem on Human Subject Networks,” Science 313(5788), 824-827.

Kearns, M., Judd, S., Tan, J. and Wortman, J. (2009): "Behavioral Experiments on Biased Voting in Networks,” PNAS 106(5), 1347-1352.

Kelso A.S., and Crawford, V.P. (1982): "Job Matching, Coalition Formation, and Gross Substitutes," Econometrica 50(6), 1483-1504.

Keser, C., Ehrhart, K. and Berninghaus, S.K. (1998): "Coordination and Local Interaction: Experimental Evidence,” Economics Letters 58(3), 269-275.

Kirchkamp, O. and Nagel, R. (2007): "Naive Learning and Cooperation in Network Experiments," Games and Economic Behavior 58(2), 269-292.

Kranton, R.E. and Minehart, D.F. (2001): “A Theory of Buyer-Seller Networks,” American Economic Review 91(3), 485-508.

Mengel, F. (2010): “Learning Across Games,” Games and Economic Behavior 74, 601-619.

Montgomery, J.D. (1991): "Social Networks and Labor-Market Outcomes: Toward an Economic Analysis,” The American Economic Review, 81(5), 1408-1418.

Newman, M.E., Strogatz, S.H. and Watts D.J. (2001): "Random graphs with arbitrary degree distributions and their applications,” Physical Review E, 64:026118.

Peski, M. (2010): “Generalized Risk-Dominance and Asymmetric Dynamics,” Journal of Economic Theory 145, 216-248.

Riedl, A. and Ule, A. (2002): "Exclusion and Cooperation in Social Network Experiments," mimeo, University of Amsterdam.

Rosenkranz, S., and Weitzel, U. (2012): "Network Structure and Strategic Investments: An Experimental Analysis," Games and Economic Behavior 75, 898-920.

Roth, A.E. (1984): “The Evolution of the Labor Market for Medical Interns and Residents: A Case Study in Game Theory,” Journal of Political Economy 92(6), 991-1016.

Roth, A. and Sotomayor, M. (1989): Two Sided Matching, Econometric Society Monographs No. 18: Cambridge University Press, Cambridge, UK.

Stolte, J. and Emerson, R. (1977): "Structural Inequality: Position and Power in Network Structures" in Behavioral Theory in Sociology (pp. 117-38), ed. R. Hamblin and J. Kunkel, New Brunswick, NJ: Transaction Books.

Sundararajan, A. (2007): "Local Network Effects and Complex Network Structure," The B.E. Journal of Theoretical Economics 7(1), Article 46.

Van Huyck, J.B., Battalio, R.C. and Beil, R.O. (1990): “Tacit Coordination Games, Strategic Uncertainty, and Coordination Failure,” The American Economic Review 80(1), 234-248.

Von Neumann, J. and Morgenstern, O. (1972): Theory of Games and Economic Behavior, Princeton: Princeton University Press.

Willer, D. (1999): Network Exchange Theory, New York: Praeger. 


\section{Tables and Figures}

\section{Table 1: Equilibria in Experiment 1}

I) With complete information

\begin{tabular}{|c|c|c|c|}
\hline & Network & Active nodes & Inactive nodes \\
\hline \multirow{10}{*}{ Substitutes } & \multirow{3}{*}{ Orange } & A, C, E & B, D \\
\hline & & $\mathrm{B}, \mathrm{E}$ & A, C, D \\
\hline & & A, D & $B, C, E$ \\
\hline & \multirow{4}{*}{ Green } & A, C, E & B, D \\
\hline & & B, D & A, C, E \\
\hline & & $\mathrm{B}, \mathrm{E}$ & A, C, D \\
\hline & & A, D & $\mathrm{B}, \mathrm{C}, \mathrm{E}$ \\
\hline & \multirow{3}{*}{ Purple } & A, C, D & $\mathrm{B}, \mathrm{E}$ \\
\hline & & A, C, E & $\mathrm{B}, \mathrm{D}$ \\
\hline & & $\mathrm{B}, \mathrm{E}$ & A, C, D \\
\hline \multirow{4}{*}{ Complements } & Oranøe & B, C, D & $\mathrm{A}, \mathrm{E}$ \\
\hline & Uidange & - & A, B, C, D, E \\
\hline & Green & - & $\mathrm{A}, \mathrm{B}, \mathrm{C}, \mathrm{D}, \mathrm{E}$ \\
\hline & Purple & - & $\mathrm{A}, \mathrm{B}, \mathrm{C}, \mathrm{D}, \mathrm{E}$ \\
\hline \multicolumn{4}{|c|}{ II) With incomplete information } \\
\hline & $\begin{array}{l}\text { Probability of the } \\
\text { Orange network }\end{array}$ & Active degrees & Inactive degrees \\
\hline \multirow{2}{*}{ Substitutes } & 0.2 & 1 & 2,3 \\
\hline & 0.8 & 1,2 & 3 \\
\hline \multirow{5}{*}{ Complements } & 0.2 & - & $1,2,3$ \\
\hline & \multirow{2}{*}{0.8} & - & 1, 2, 3 \\
\hline & & 2,3 & 1 \\
\hline & 0.95 (addendum to & - & $1,2,3$ \\
\hline & Experiment 1) & 2,3 & 1 \\
\hline
\end{tabular}


Table 2: Equilibria in Experiment 2

I) With complete information

\begin{tabular}{lll}
\hline Network & Active nodes & Inactive nodes \\
\hline \multirow{2}{*}{ Blue } & B, C, D, E & A $~$ \\
& - & A, B, C, D, E \\
\multirow{2}{*}{ Red } & C, D, E & A, B \\
& - & A, B, C, D, E \\
\multirow{2}{*}{ Brown } & B, C, D, E & A \\
& - & A, B, C, D, E \\
\multirow{2}{*}{ II) With incomplete information } & \\
\hline \multirow{2}{*}{$\begin{array}{l}\text { Probability of the Blue } \\
\text { network }\end{array}$} & Active degrees & \multicolumn{1}{c}{ Inactive degrees } \\
\multirow{2}{*}{0.2} & - & $1,2,3$ \\
& 2,3 & 1 \\
\hline \multirow{2}{*}{0.8} & - & $1,2,3$ \\
& 2,3 & 1 \\
\hline
\end{tabular}

Table 3: Network Characteristics in Experiment 3

\begin{tabular}{ccccc}
\hline & & Network 1 & Network 2 & Network 3 \\
\hline Degree & degree $=1$ & 8 & 4 & 4 \\
distribution & degree $=2$ & 6 & 4 & 4 \\
(\# nodes) & degree $=3$ & 4 & 6 & 6 \\
& degree $=4$ & 2 & 6 & 6 \\
\hline Number of links & 20 & 27 & 27 \\
\hline (Global) Clustering coefficient & 0 & 0 & 0.31 \\
\hline
\end{tabular}

Table 4: Equilibria in Experiment 3

\begin{tabular}{lll}
\hline Network & Active degrees & Inactive degrees \\
\hline \multirow{2}{*}{ Network 1} & - & $1,2,3,4$ \\
& $2,3,4$ & 1 \\
\hline \multirow{2}{*}{ Network 2} & - & $1,2,3,4$ \\
& $2,3,4$ & 1 \\
\hline \multirow{2}{*}{ Network 3 } & - & $1,2,3,4$ \\
& $2,3,4$ & 1 \\
\hline
\end{tabular}


Table 5: Complete information in Experiment 1

Frequencies of activity by treatment, network and player position

\begin{tabular}{|c|c|c|c|c|c|c|c|}
\hline & \multicolumn{2}{|c|}{ Orange } & \multicolumn{2}{|c|}{ Green } & \multicolumn{2}{|c|}{ Purple } \\
\hline & & $\begin{array}{l}\text { Active } \\
\text { (\%) }\end{array}$ & Total & $\begin{array}{c}\text { Active } \\
\text { (\%) }\end{array}$ & Total & $\begin{array}{l}\text { Active } \\
(\%)\end{array}$ & Total \\
\hline \multirow{6}{*}{ Substitutes } & A & $\begin{array}{c}88 \\
(94.62)\end{array}$ & 93 & $\begin{array}{c}96 \\
(91.43)\end{array}$ & 105 & $\begin{array}{c}113 \\
(92.62)\end{array}$ & 122 \\
\hline & B & $\begin{array}{c}8 \\
(8.60)\end{array}$ & 93 & $\begin{array}{c}16 \\
(15.24)\end{array}$ & 105 & $\begin{array}{c}6 \\
(4.92)\end{array}$ & 122 \\
\hline & C & $\begin{array}{c}63 \\
(67.74)\end{array}$ & 93 & $\begin{array}{c}70 \\
(66.67)\end{array}$ & 105 & $\begin{array}{c}115 \\
(94.26)\end{array}$ & 122 \\
\hline & $\mathrm{D}$ & $\begin{array}{c}10 \\
(10.75)\end{array}$ & 93 & $\begin{array}{c}18 \\
(17.14)\end{array}$ & 105 & $\begin{array}{c}22 \\
(18.03)\end{array}$ & 122 \\
\hline & E & $\begin{array}{c}85 \\
(91.40) \\
\end{array}$ & 93 & $\begin{array}{c}99 \\
(94.29) \\
\end{array}$ & 105 & $\begin{array}{c}112 \\
(91.80) \\
\end{array}$ & 122 \\
\hline & Total & $\begin{array}{c}254 \\
(54.62) \\
\end{array}$ & 465 & $\begin{array}{c}299 \\
(56.95) \\
\end{array}$ & 525 & $\begin{array}{c}368 \\
(60.33) \\
\end{array}$ & 610 \\
\hline \multirow{6}{*}{ Complements } & A & $\begin{array}{c}4 \\
(3.51) \\
\end{array}$ & 114 & $\begin{array}{c}1 \\
(0.95)\end{array}$ & 105 & $\begin{array}{c}1 \\
(0.99)\end{array}$ & 101 \\
\hline & B & $\begin{array}{c}85 \\
(74.56) \\
\end{array}$ & 114 & $\begin{array}{c}4 \\
(3.81) \\
\end{array}$ & 105 & $\begin{array}{c}13 \\
(12.87) \\
\end{array}$ & 101 \\
\hline & $\mathrm{C}$ & $\begin{array}{c}83 \\
(72.81)\end{array}$ & 114 & $\begin{array}{c}11 \\
(10.48)\end{array}$ & 105 & $\begin{array}{c}1 \\
(0.99)\end{array}$ & 101 \\
\hline & $\mathrm{D}$ & $\begin{array}{c}85 \\
(74.56)\end{array}$ & 114 & $\begin{array}{c}2 \\
(1.90)\end{array}$ & 105 & $\begin{array}{c}5 \\
(4.95) \\
\end{array}$ & 101 \\
\hline & $\mathrm{E}$ & $\begin{array}{c}6 \\
(5.26) \\
\end{array}$ & 114 & $\begin{array}{c}1 \\
(0.95)\end{array}$ & 105 & $\begin{array}{c}1 \\
(0.99)\end{array}$ & 101 \\
\hline & Total & $\begin{array}{c}263 \\
(46.14)\end{array}$ & 570 & $\begin{array}{c}19 \\
(3.62)\end{array}$ & 525 & $\begin{array}{c}21 \\
(4.16)\end{array}$ & 505 \\
\hline
\end{tabular}

Estimated activity rates by treatment, network and player position (at period $=20$ and average risk level)

\begin{tabular}{ccccccc}
\hline & & Substitutes & & \multicolumn{3}{c}{ Complements } \\
\hline Position & Orange & Green & Purple & Orange & Green & Purple \\
\hline \multirow{2}{*}{$\mathrm{A}$} & $0.989^{* * *}$ & $0.975^{* * *}$ & $0.977^{* * *}$ & 0.010 & 0.000 & 0.000 \\
& $(0.007)$ & $(0.014)$ & $(0.013)$ & $(0.007)$ & $(0.000)$ & $(0.000)$ \\
\hline \multirow{2}{*}{$\mathrm{B}$} & 0.011 & $0.048^{*}$ & 0.013 & $0.843^{* * *}$ & 0.007 & $0.050^{*}$ \\
& $(0.008)$ & $(0.025)$ & $(0.009)$ & $(0.050)$ & $(0.006)$ & $(0.027)$ \\
\hline \multirow{2}{*}{$\mathrm{C}$} & $0.767^{* * *}$ & $0.754^{* * *}$ & $0.990^{* * *}$ & $0.746^{* * *}$ & 0.010 & 0.000 \\
& $(0.076)$ & $(0.079)$ & $(0.008)$ & $(0.067)$ & $(0.009)$ & $(0.000)$ \\
\hline \multirow{2}{*}{$\mathrm{D}$} & 0.023 & $0.091^{* *}$ & $0.097^{* *}$ & $0.813^{* * *}$ & 0.001 & 0.006 \\
& $(0.014)$ & $(0.039)$ & $(0.040)$ & $(0.057)$ & $(0.002)$ & $(0.007)$ \\
\hline \multirow{2}{*}{$\mathrm{E}$} & $0.978^{* * *}$ & $0.987^{* * *}$ & $0.970^{* * *}$ & 0.000 & 0.001 & 0.000 \\
& $(0.014)$ & $(0.010)$ & $(0.016)$ & $(0.001)$ & $(0.002)$ & $(0.000)$ \\
\hline
\end{tabular}

$* * *, * *, *$ denote significance at $1 \%, 5 \%$ and $10 \%$ levels, respectively, two-tailed tests 
Table 6: - Incomplete information in Experiment 1

Frequencies of activity by connectivity ( $p$ ) and degree

\begin{tabular}{|c|c|c|c|c|c|}
\hline & & \multicolumn{2}{|c|}{$p=0.2$} & \multicolumn{2}{|c|}{$p=0.8$} \\
\hline & & $\begin{array}{c}\text { Active } \\
\text { (\%) }\end{array}$ & Total & $\begin{array}{c}\text { Active } \\
\text { (\%) }\end{array}$ & Total \\
\hline \multirow{4}{*}{ Substitutes } & degree $=1$ & $\begin{array}{c}731 \\
(94.81) \\
\end{array}$ & 771 & $\begin{array}{c}628 \\
(92.90) \\
\end{array}$ & 676 \\
\hline & degree $=2$ & $\begin{array}{c}156 \\
(28.16) \\
\end{array}$ & 554 & $\begin{array}{c}225 \\
(59.52) \\
\end{array}$ & 378 \\
\hline & degree $=3$ & $\begin{array}{c}3 \\
(1.09)\end{array}$ & 275 & $\begin{array}{c}55 \\
(10.07)\end{array}$ & 546 \\
\hline & Total & $\begin{array}{c}890 \\
(55.63)\end{array}$ & 1600 & $\begin{array}{c}908 \\
(56.75)\end{array}$ & 1600 \\
\hline \multirow{4}{*}{ Complements } & degree $=1$ & $\begin{array}{c}15 \\
(1.97) \\
\end{array}$ & 763 & $\begin{array}{c}12 \\
(1.76)\end{array}$ & 681 \\
\hline & degree $=2$ & $\begin{array}{c}107 \\
(17.89)\end{array}$ & 598 & $\begin{array}{c}116 \\
(31.02)\end{array}$ & 374 \\
\hline & degree $=3$ & $\begin{array}{c}106 \\
(44.35)\end{array}$ & 239 & $\begin{array}{c}278 \\
(51.01)\end{array}$ & 545 \\
\hline & Total & $\begin{array}{c}228 \\
(14.25)\end{array}$ & 1600 & $\begin{array}{c}406 \\
(25.37)\end{array}$ & 1600 \\
\hline
\end{tabular}

Marginal effects of connectivity (p) by treatment and degree $(p=0.8$ vs. $p=0.2$ at period 20 and average risk level)

\begin{tabular}{ccc}
\hline & Substitutes & Complements \\
\hline \multirow{2}{*}{ degree $=1$} & 0.004 & -0.000 \\
& $(0.009)$ & $(0.001)$ \\
\hline \multirow{2}{*}{ degree $=2$} & $0.547^{* * *}$ & $0.111^{* *}$ \\
& $(0.083)$ & $(0.056)$ \\
\hline \multirow{2}{*}{ degree $=3$} & $0.024^{* *}$ & $0.233^{*}$ \\
& $(0.010)$ & $(0.132)$ \\
\hline
\end{tabular}

Marginal effects of degree by treatment and connectivity ( $p$ ) (at period 20 and average risk level)

\begin{tabular}{|c|c|c|c|}
\hline & & Substitutes & Complements \\
\hline \multirow{2}{*}{ degree $=2$ vs. degree $=1$} & $p=0.2$ & $\begin{array}{c}-0.816^{* * *} \\
(0.041)\end{array}$ & $\begin{array}{c}0.041^{* *} \\
(0.017)\end{array}$ \\
\hline & $p=0.8$ & $\begin{array}{c}-0.273^{* * *} \\
(0.064)\end{array}$ & $\begin{array}{c}0.153 * * * \\
(0.053)\end{array}$ \\
\hline \multirow{2}{*}{ degree $=3$ vs. degree $=1$} & $p=0.2$ & $\begin{array}{c}-0.980 * * * \\
(0.007)\end{array}$ & $\begin{array}{c}0.328 * * * \\
(0.091)\end{array}$ \\
\hline & $p=0.8$ & $\begin{array}{c}-0.961 * * * \\
(0.009)\end{array}$ & $\begin{array}{c}0.562 * * * \\
(0.095)\end{array}$ \\
\hline \multirow{2}{*}{ degree $=3$ vs. degree $=2$} & $p=0.2$ & $\begin{array}{c}-0.164 * * * \\
(0.045)\end{array}$ & $\begin{array}{c}0.287 * * * \\
(0.079)\end{array}$ \\
\hline & $p=0.8$ & $\begin{array}{c}-0.687 * * * \\
(0.063)\end{array}$ & $\begin{array}{c}0.409 * * * \\
(0.061)\end{array}$ \\
\hline
\end{tabular}

$* * *, * *, *$ denote significance at $1 \%, 5 \%$ and $10 \%$ levels, respectively, two-tailed tests 
Table 7. Experiment 1

Estimated activity rates by connectivity (p), degree and period (at average risk level)

\begin{tabular}{ccccc}
\hline & & $p=0.80$ & $p=0.95$ & $p=1$ \\
\hline \multirow{3}{*}{ degree $=2$} & period $=20$ & $0.153^{* * *}$ & 0.805 & 0.746 \\
& & $(0.053)$ & $(0.072)$ & $(0.067)$ \\
\cline { 2 - 5 } & \multirow{2}{*}{ period $=40$} & $0.007^{* * *}$ & 0.416 & $0.747^{* *}$ \\
& & $(0.004)$ & $(0.131)$ & $(0.102)$ \\
\hline \multirow{2}{*}{ degree $=3$} & period $=20$ & $0.563^{* * *}$ & 0.919 & 0.828 \\
& & $(0.095)$ & $(0.034)$ & $(0.045)$ \\
\cline { 2 - 5 } & \multirow{2}{*}{ period $=40$} & $0.012^{* * *}$ & 0.388 & $0.685^{* *}$ \\
& & $(0.006)$ & $(0.117)$ & $(0.090)$ \\
\hline
\end{tabular}

Estimated trends of activity rates by connectivity (p) and degree (at period 20 and average risk level)

\begin{tabular}{cccc}
\hline degree $=2$ & -0.008 & -0.014 & $0.000^{* * *}$ \\
& $(0.003)$ & $(0.004)$ & $(0.000)$ \\
\hline \multirow{2}{*}{ degree $=3$} & $-0.047 * * *$ & -0.011 & -0.006 \\
& $(0.008)$ & $(0.004)$ & $(0.002)$ \\
\hline
\end{tabular}

$* * *, * *, *$ denote significant difference respect to treatment $\mathrm{p}=0.95$ at $1 \%, 5 \%$ and $10 \%$ levels, respectively, two-tailed tests

Note that $\mathrm{p}=1$ means the orange network in the treatment of complete information. 
Table 8. Complete Information in Experiment 2

Frequencies of activity by network and player position

\begin{tabular}{|c|c|c|c|c|c|c|}
\hline \multirow[b]{2}{*}{ Position } & \multicolumn{2}{|c|}{ Blue } & \multicolumn{2}{|c|}{ Red } & \multicolumn{2}{|c|}{ Brown } \\
\hline & $\begin{array}{c}\text { Active } \\
\text { (\%) }\end{array}$ & Total & $\begin{array}{c}\text { Active } \\
\text { (\%) }\end{array}$ & Total & $\begin{array}{c}\text { Active } \\
\text { (\%) }\end{array}$ & Total \\
\hline A & $\begin{array}{c}0 \\
(0.00) \\
\end{array}$ & 112 & $\begin{array}{c}1 \\
(0.93) \\
\end{array}$ & 108 & $\begin{array}{c}2 \\
(2.00) \\
\end{array}$ & 100 \\
\hline B & $\begin{array}{c}104 \\
(92.86)\end{array}$ & 112 & $\begin{array}{c}20 \\
(18.52)\end{array}$ & 108 & $\begin{array}{c}79 \\
(79.00)\end{array}$ & 100 \\
\hline C & $\begin{array}{c}109 \\
(97.32)\end{array}$ & 112 & $\begin{array}{c}92 \\
(85.19)\end{array}$ & 108 & $\begin{array}{c}62 \\
(62.00)\end{array}$ & 100 \\
\hline D & $\begin{array}{c}85 \\
(75.89)\end{array}$ & 112 & $\begin{array}{c}70 \\
(64.81)\end{array}$ & 108 & $\begin{array}{c}60 \\
(60.00)\end{array}$ & 100 \\
\hline $\mathrm{E}$ & $\begin{array}{c}108 \\
(96.43)\end{array}$ & 112 & $\begin{array}{c}68 \\
(62.96)\end{array}$ & 108 & $\begin{array}{c}56 \\
(56.00)\end{array}$ & 100 \\
\hline Degree $2^{\wedge}$ & $\begin{array}{c}85 \\
(75.89) \\
\end{array}$ & 112 & $\begin{array}{c}138 \\
(63.88)\end{array}$ & 216 & $\begin{array}{c}178 \\
(59.33) \\
\end{array}$ & 300 \\
\hline Degree 3 & $\begin{array}{c}321 \\
(95.54)\end{array}$ & 336 & $\begin{array}{c}92 \\
(85.19)\end{array}$ & 108 & $\begin{array}{c}79 \\
(79.00)\end{array}$ & 100 \\
\hline
\end{tabular}

Estimated activity rates by network and player position (at period 20 and average risk level)

\begin{tabular}{cccc}
\hline & Blue & Red & Brown \\
\hline \multirow{2}{*}{$\mathrm{A}$} & 0.000 & 0.000 & 0.000 \\
& $()$. & $()$. & $()$. \\
\hline \multirow{2}{*}{$\mathrm{B}$} & $0.989^{* * *}$ & $0.065^{* *}$ & $0.913^{* * *}$ \\
& $(0.007)$ & $(0.034)$ & $(0.042)$ \\
\hline \multirow{2}{*}{$\mathrm{C}$} & $0.996^{* * *}$ & $0.992^{* * *}$ & $0.581^{* * *}$ \\
& $(0.003)$ & $(0.005)$ & $(0.120)$ \\
\hline \multirow{2}{*}{$\mathrm{D}$} & $0.882^{* * *}$ & $0.753^{* * *}$ & $0.668^{* * *}$ \\
& $(0.052)$ & $(0.091)$ & $(0.109)$ \\
\hline \multirow{2}{*}{$\mathrm{E}$} & $0.999^{* * *}$ & $0.785^{* * *}$ & $0.674^{* * *}$ \\
& $(0.001)$ & $(0.085)$ & $(0.110)$ \\
\hline \multirow{2}{*}{ Degree $2^{\wedge}$} & $0.882^{* * *}$ & $0.769^{* * *}$ & $0.641^{* * *}$ \\
& $(0.052)$ & $(0.080)$ & $(0.091)$ \\
\hline \multirow{2}{*}{ Degree 3 } & $0.995^{* * *}$ & $0.992^{* * *}$ & $0.913^{* * *}$ \\
& $(0.003)$ & $(0.005)$ & $(0.042)$ \\
\hline
\end{tabular}

$* * *, * *, *$ denote significance at $1 \%, 5 \%$ and $10 \%$ levels, respectively, two-tailed tests

$\wedge$ Here we are only referring to those players with degree 2 active in the efficient equilibrium. 
Table 9. Incomplete information in Experiment 2

Frequencies of activity by connectivity ( $p$ ) and degree

\begin{tabular}{|c|c|c|c|c|}
\hline & \multicolumn{2}{|c|}{$p=0.2$} & \multicolumn{2}{|c|}{$p=0.8$} \\
\hline & $\begin{array}{c}\text { Active } \\
(\%)\end{array}$ & Total & $\begin{array}{l}\text { Active } \\
(\%)\end{array}$ & Total \\
\hline degree $=1$ & $\begin{array}{c}25 \\
(7.81)\end{array}$ & 320 & $\begin{array}{c}23 \\
(7.19)\end{array}$ & 320 \\
\hline degree $=2$ & $\begin{array}{c}603 \\
(72.48)\end{array}$ & 832 & $\begin{array}{c}339 \\
(79.21)\end{array}$ & 428 \\
\hline degree $=3$ & $\begin{array}{c}425 \\
(94.87)\end{array}$ & 448 & $\begin{array}{c}821 \\
(96.36)\end{array}$ & 852 \\
\hline Total & $\begin{array}{c}1053 \\
(65.81)\end{array}$ & 1600 & $\begin{array}{c}1183 \\
(73.94)\end{array}$ & 1600 \\
\hline
\end{tabular}

Marginal effect of connectivity ( $p$ ) by degree and period $(p=0.8 v s . p=0.2$ at average risk level)

\begin{tabular}{ccc}
\hline & period $=20$ & period $=40$ \\
\hline \multirow{2}{*}{ degree $=1$} & 0.001 & -0.001 \\
& $(0.005)$ & $(0.002)$ \\
\hline \multirow{2}{*}{ degree $=2$} & -0.041 & $0.272^{*}$ \\
& $(0.060)$ & $0.151)$ \\
\hline \multirow{2}{*}{ degree $=3$} & -0.000 & 0.004 \\
& $(0.001)$ & $(0.004)$ \\
\hline
\end{tabular}

Marginal effect of degree by connectivity ( $p$ ) (at period 20 and average risk level)

\begin{tabular}{|c|c|c|}
\hline \multirow{2}{*}{ degree $=2$ vs. degree $=1$} & $\mathrm{p}=0.2$ & $\begin{array}{c}0.943 * * * \\
(0.030) \\
\end{array}$ \\
\hline & $\mathrm{p}=0.8$ & $\begin{array}{c}0.900 * * * \\
(0.049)\end{array}$ \\
\hline \multirow{2}{*}{ degree $=3$ vs. degree $=1$} & $\mathrm{p}=0.2$ & $\begin{array}{c}0.995 * * * \\
(0.003)\end{array}$ \\
\hline & $\mathrm{p}=0.8$ & $\begin{array}{c}0.994 * * * \\
(0.004)\end{array}$ \\
\hline \multirow{2}{*}{ degree $=3$ vs. degree $=2$} & $\mathrm{p}=0.2$ & $\begin{array}{l}0.052^{*} \\
(0.031)\end{array}$ \\
\hline & $\mathrm{p}=0.8$ & $\begin{array}{l}0.093^{*} \\
(0.051)\end{array}$ \\
\hline
\end{tabular}

***, **,* denote significance at $1 \%, 5 \%, 10 \%$ levels, respectively, two-tailed tests 
Table 10. Experiment 3

Frequencies of activity by network and degree

\begin{tabular}{ccccccc}
\hline & \multicolumn{2}{c}{ Network 1 } & \multicolumn{2}{c}{ Network 2 } & \multicolumn{2}{c}{ Network 3 } \\
\hline & $\begin{array}{c}\text { Active } \\
(\%)\end{array}$ & Total & $\begin{array}{c}\text { Active } \\
(\%)\end{array}$ & Total & $\begin{array}{c}\text { Active } \\
(\%)\end{array}$ & Total \\
\hline degree $=1$ & $\begin{array}{c}26 \\
(2.71)\end{array}$ & 960 & $\begin{array}{c}16 \\
(3.33)\end{array}$ & 480 & $\begin{array}{c}34 \\
(7.17)\end{array}$ & 474 \\
\hline degree $=2$ & $\begin{array}{c}177 \\
(24.58)\end{array}$ & 720 & $\begin{array}{c}423 \\
(88.13)\end{array}$ & 480 & $\begin{array}{c}434 \\
(92.93)\end{array}$ & 467 \\
\hline degree $=3$ & $\begin{array}{c}291 \\
(60.62)\end{array}$ & 480 & $\begin{array}{c}720 \\
(100)\end{array}$ & 720 & $\begin{array}{c}707 \\
(99.58)\end{array}$ & 710 \\
\hline degree $=4$ & $\begin{array}{c}795 \\
(81.25)\end{array}$ & 240 & $\begin{array}{c}719 \\
(99.86)\end{array}$ & 720 & $\begin{array}{c}709 \\
(100)\end{array}$ & 709 \\
\hline
\end{tabular}

Marginal effect of network by degree (at period 20 and average risk level)

\begin{tabular}{cccc}
\hline & Network 2 vs. Network 1 & Network 3 vs. Network 1 & Network 3 vs. Network 2 \\
\hline \multirow{2}{*}{ degree $=1$} & 0.004 & $0.010^{*}$ & 0.006 \\
& $(0.004)$ & $(0.006)$ & $(0.006)$ \\
\hline \multirow{2}{*}{ degree $=2$} & $0.832^{* * *}$ & $0.836^{* * *}$ & 0.004 \\
& $(0.038)$ & $(0.037)$ & $(0.014)$ \\
\hline \multirow{2}{*}{ degree $=3$} & $0.284^{* * *}$ & $0.284^{* * *}$ & -0.001 \\
& $(0.060)$ & $(0.060)$ & $(0.000)$ \\
\hline \multirow{2}{*}{ degree $=4$} & $0.051^{* * *}$ & $0.051^{* * *}$ & 0.000 \\
& $(0.019)$ & $(0.019)$ & $(0.000)$ \\
\hline
\end{tabular}

***, **,* denote significance at $1 \%, 5 \%$ and $10 \%$ levels, respectively, two-tailed tests 
Figure 1: The networks

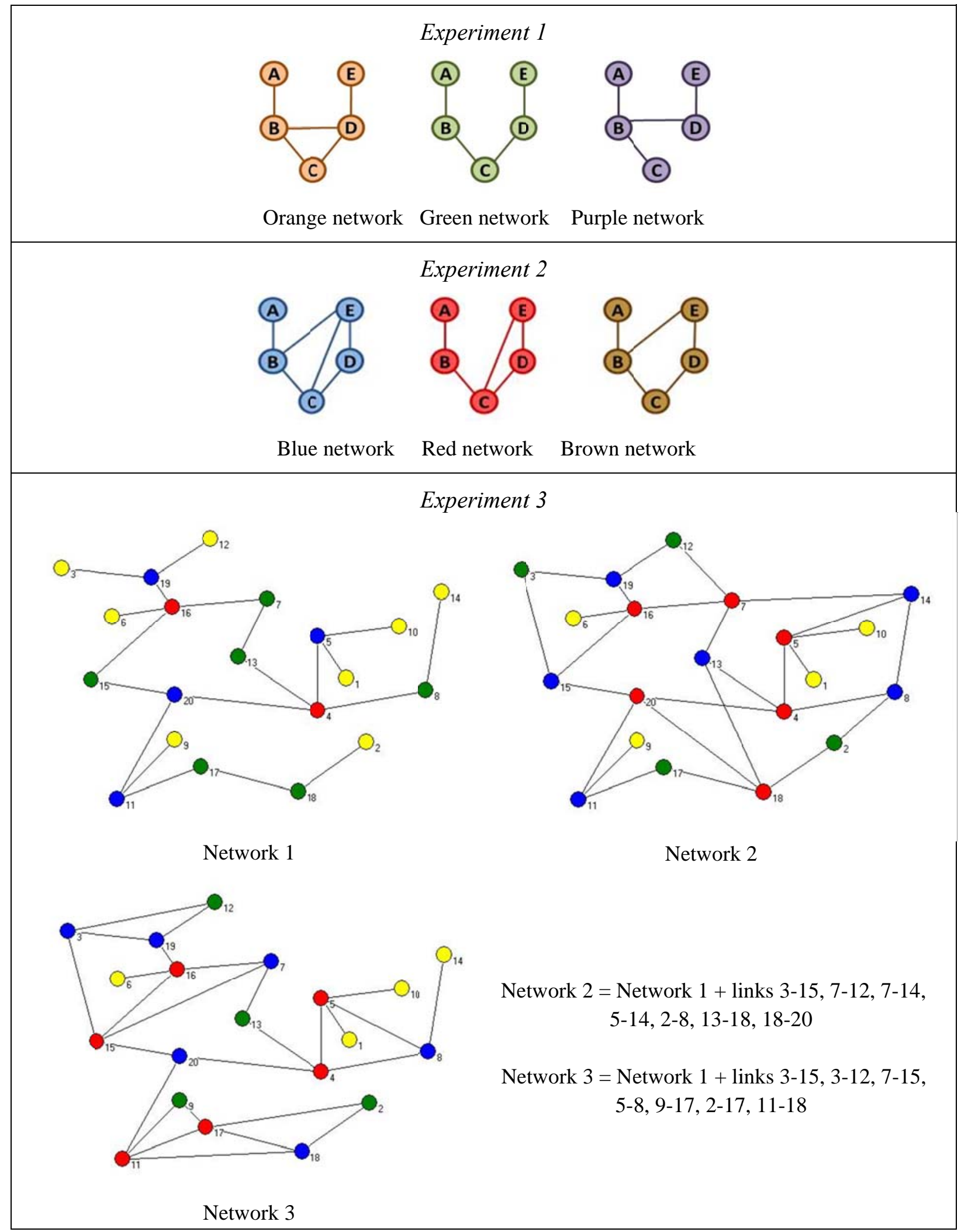


Figure 2: Relative frequency of active choices across periods, by network player position and treatment - Experiment 1, Complete information
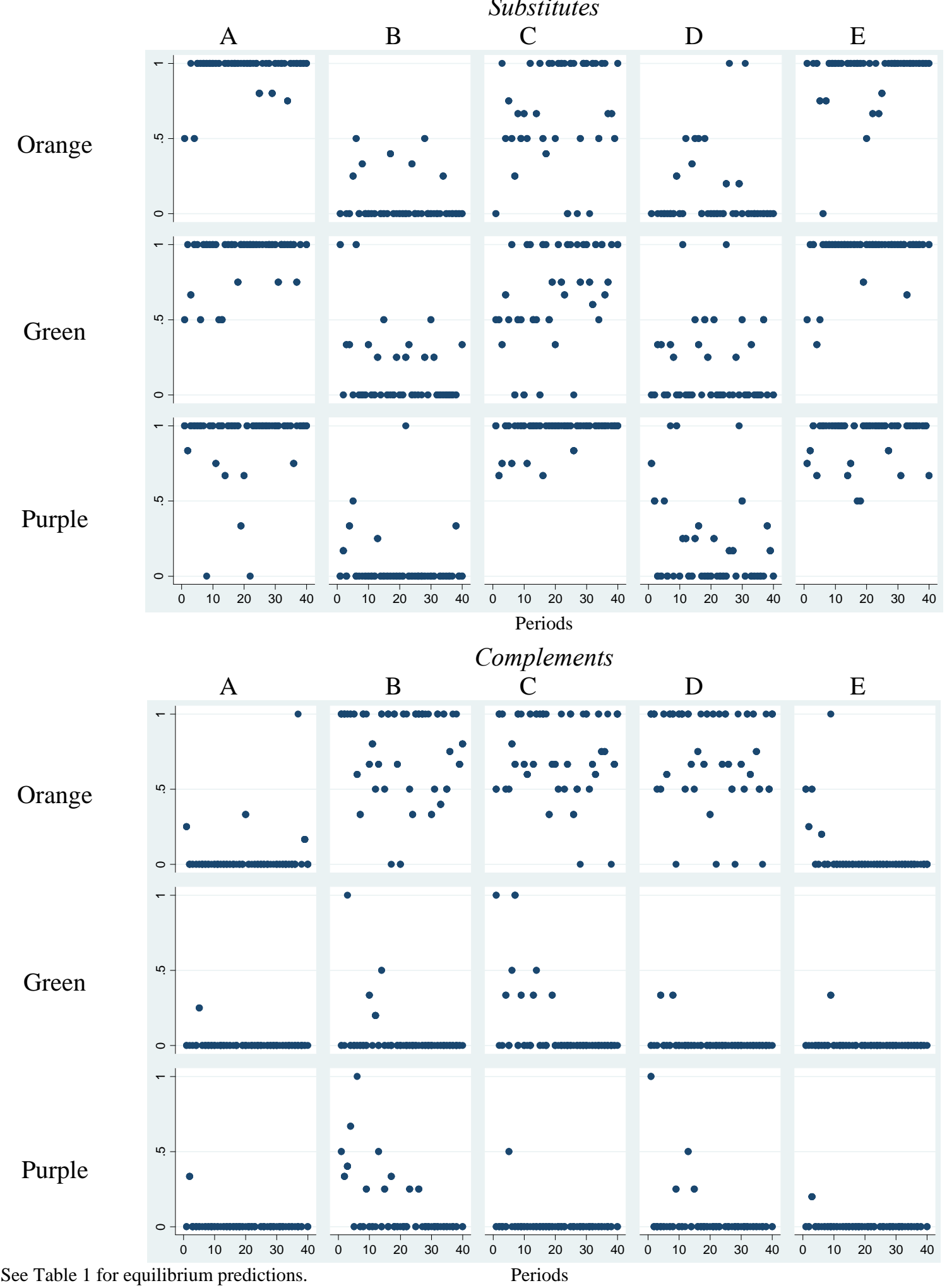
Figure 3: Relative frequencies of choices by degree, games, and $p$ Incomplete information, Experiment 1

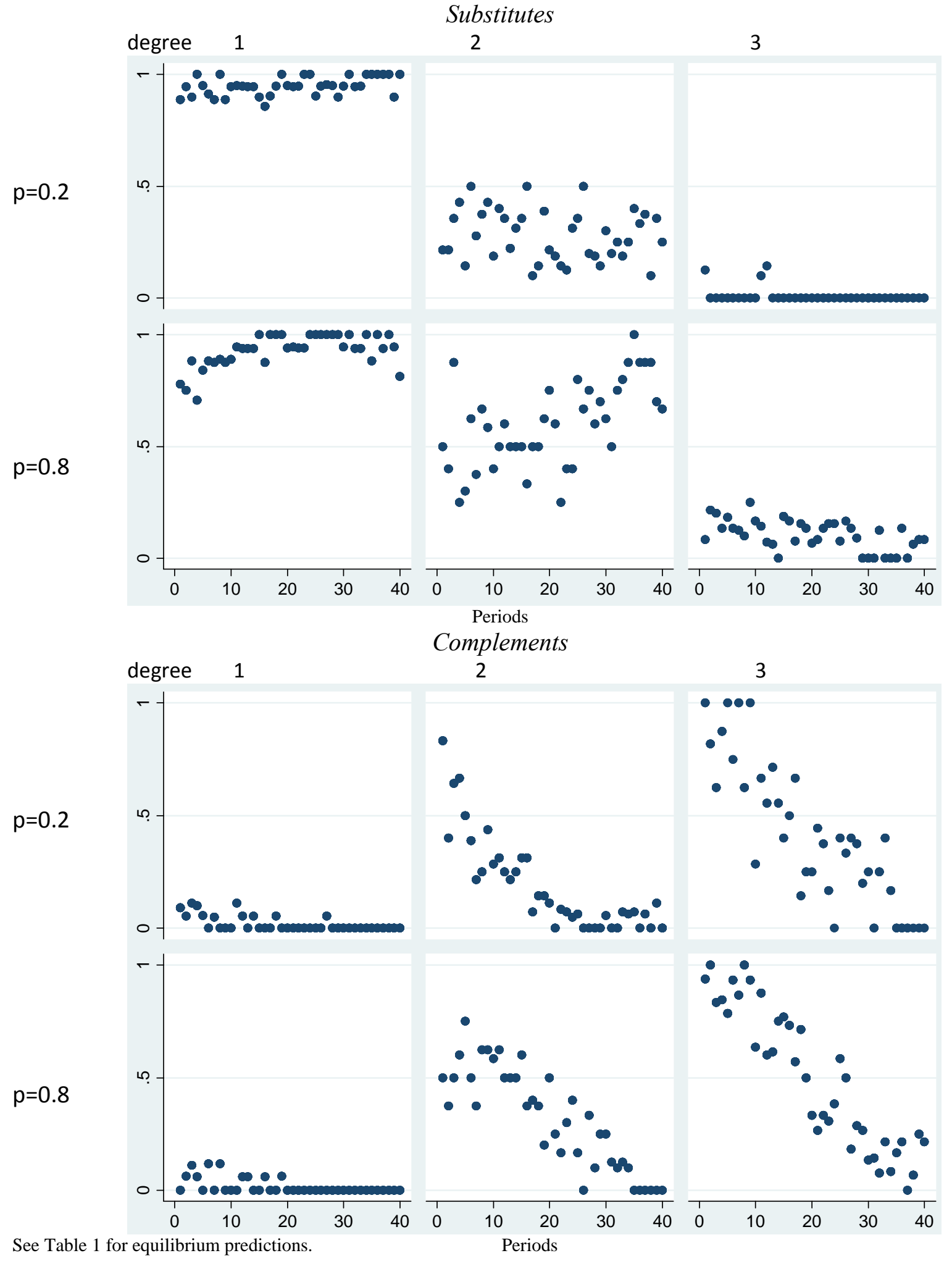


Figure 4. Observed activity probabilities, by degree and $p$, Experiment 1

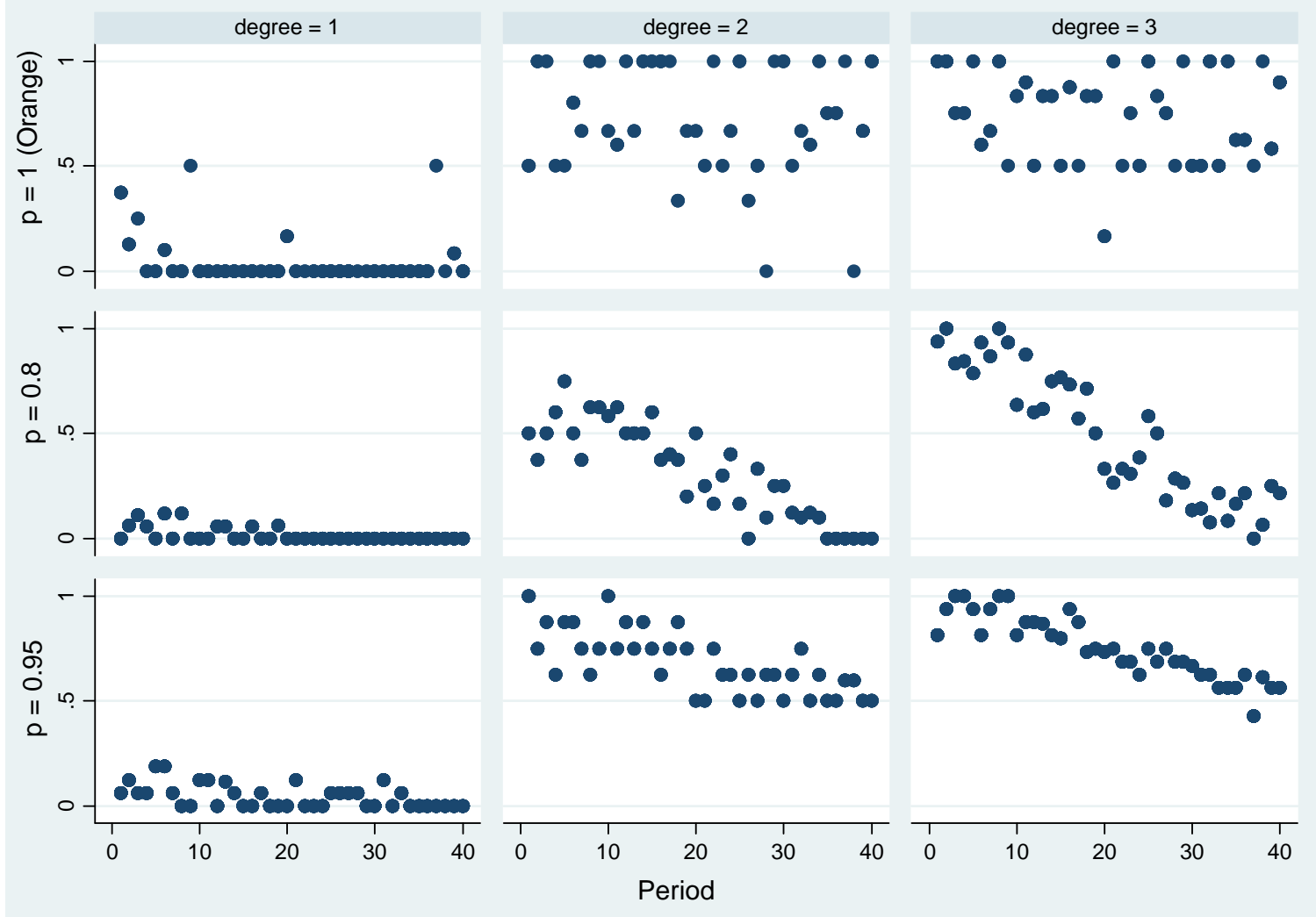

See Table 1 for equilibrium predictions. 
Figure 5. Observed probabilities of being active, by network and position in Experiment 2, Complete information

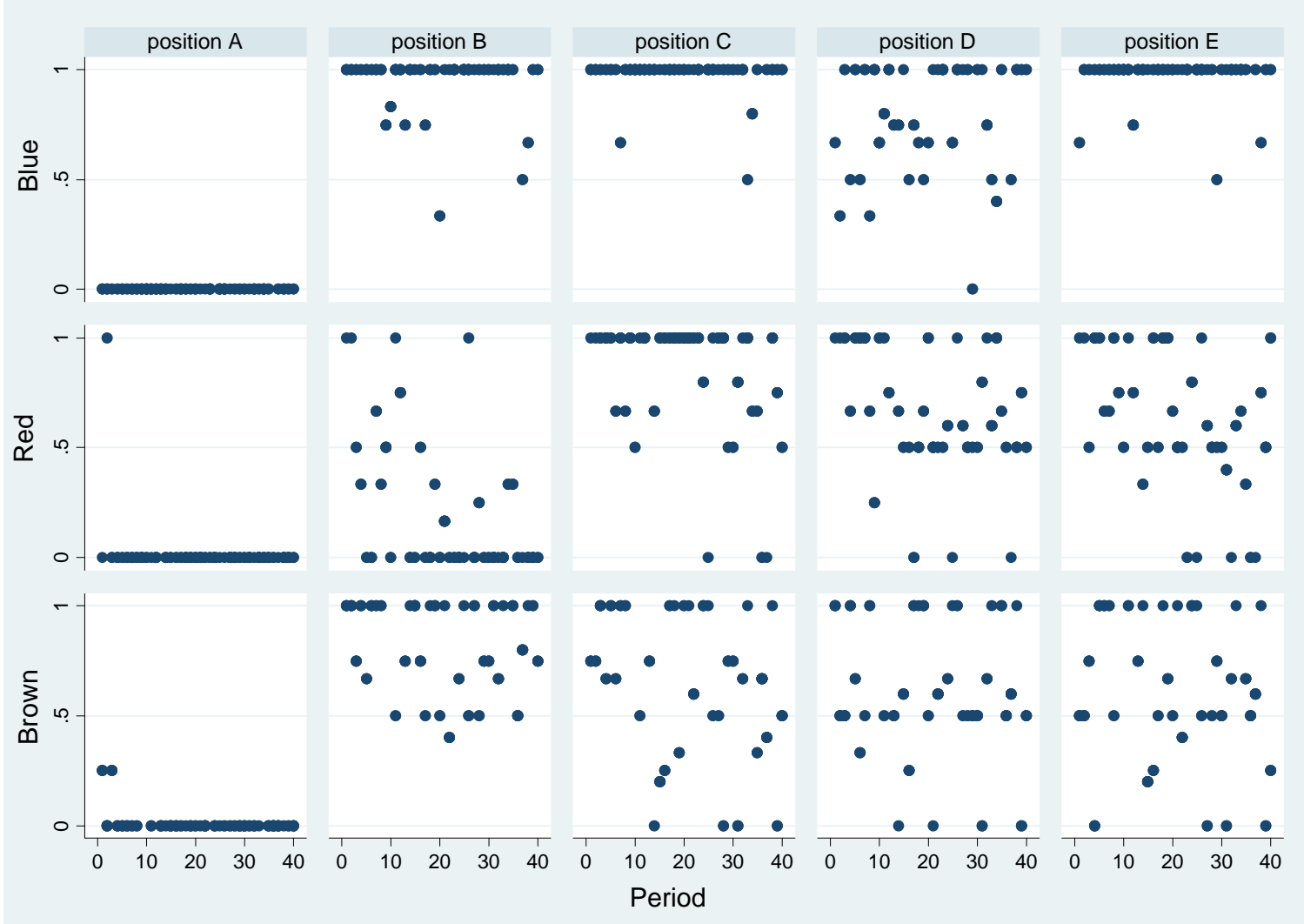

See Table 2 for equilibrium predictions. 
Figure 6. Observed probabilities of being active, by connectivity and degree Experiment 2
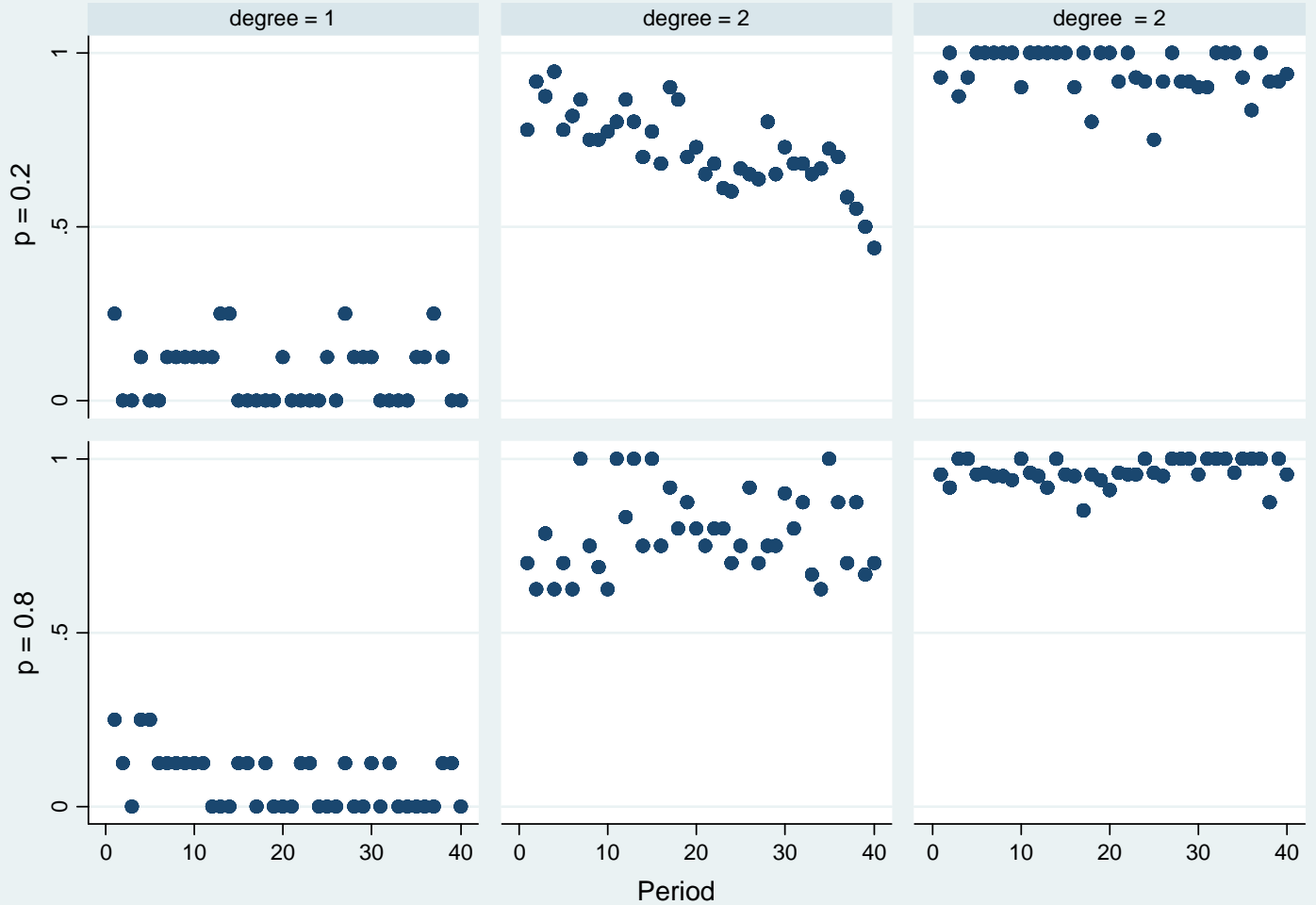

See Table 2 for equilibrium predictions. 
Figure 7. Observed probabilities of being active, by network and degree Experiment 3

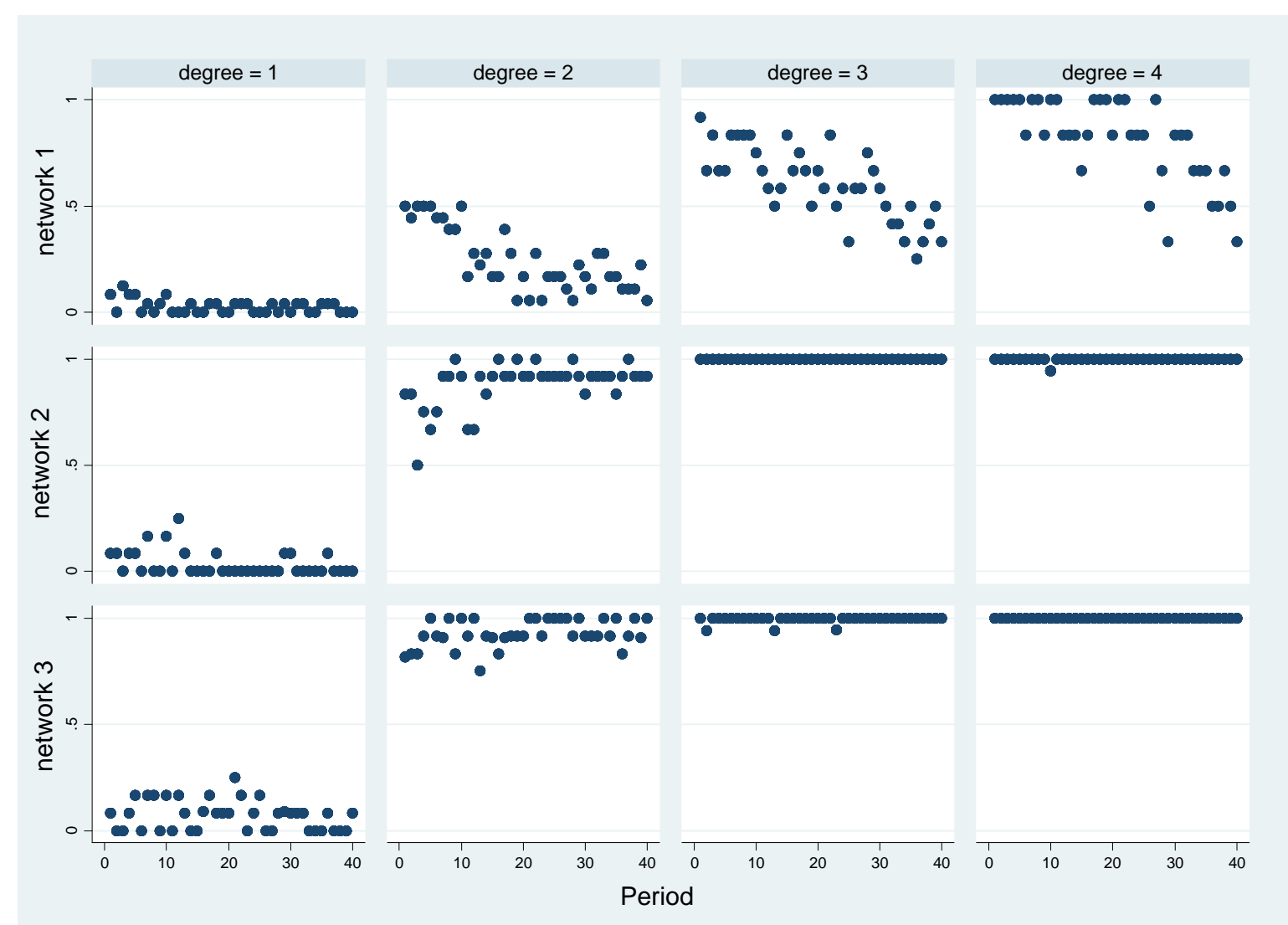

See Table 4 for equilibrium predictions. 


\section{Appendix: Proofs}

\section{Complete information, 5-player networks}

Let $s_{i} \in\{0,1\} i \in\{A, B, C, D, E\}$ be the action of the player in position $i$ where 1 means to be active 0 means to be inactive. Then a strategy profile is give by $s=\left(s_{A}, s_{B}, s_{C}, s_{D}, s_{E}\right)$. Let be $N_{i}$ the set of player that have a link to player $i$ and $n_{i}=\left|N_{i}\right|$. Let $\pi_{i}\left(s_{i}, s_{-i}\right)$ be the expected payoff of the player in position $i$.

Proposition 1. Consider the scenario of strategic substitutes and complete information of Experiment 1. In the Orange network the pure-strategy Nash equilibria are: $(1,0,1,0,1)$, $(1,0,0,1,0)$, and $(0,1,0,0,1)$. In the Green network the pure-strategy Nash equilibria are $(1,0,1,0,1),(0,1,0,1,0),(1,0,0,1,0)$, and $(0,1,0,0,1)$. In the Purple network the pure-strategy Nash equilibria are $(1,0,1,0,1),(1,0,1,1,0)$, and $(0,1,0,0,1)$. All these equilibria are strict.

Proof: It suffices to prove the following claim: In a Nash equilibrium (i) $s_{i}=1$ if and only if, $\forall j \in N_{i}, s_{j}=0$; and (ii) $s_{i}=0$ if and only if $\exists j \in N_{i}$ s.t. $s_{j}=1$. Then the result directly follows. To prove the claim, assume a Nash equilibrium where $\forall j \in N_{i}, s_{j}=0$. Then the best response of player $i$ is $s_{i}=1$ because $\pi_{i}\left(0, s_{-i}\right)=0$ and $\pi_{i}\left(1, s_{-i}\right)=50$. Assume a Nash equilibrium where $\exists j \in N_{i}$ s.t. $s_{j}=1$, then the best response of player $i$ is $s_{i}=0$ because $\pi_{i}\left(0, s_{-i}\right)=100$ and $\pi_{i}\left(1, s_{-i}\right)=50$. Assume a Nash equilibrium where $s_{i}=1$ and $\exists j \in N_{i}$ s.t. $s_{j}=1$, then the best response of player $i$ is $s_{i}=0$ because $\pi_{i}\left(0, s_{-i}\right)=100$ and $\pi_{i}\left(1, s_{-i}\right)=50$, a contradiction. Assume a Nash equilibrium where $s_{i}=0$ and $\forall j \in N_{i}, s_{j}=0$, then the best response of player $i$ is $s_{i}=1$ because $\pi_{i}\left(0, s_{-i}\right)=0$ and $\pi_{i}\left(1, s_{-i}\right)=50$, a contradiction. It is straightforward to see that all the equilibria are strict. QED

Proposition 2. Consider the scenario of strategic complements and complete information of Experiment 1. In the Orange network there are two pure-strategy Nash equilibria: $(0,0,0,0,0)$, and $(0,1,1,1,0)$. In the Green and Purple networks there is a unique Nash equilibrium: $(0,0,0,0,0)$. All these equilibria are strict.

Proof: We first prove the following claim: $s_{i}=1$ is a best response if and only if $\sum_{j \in N_{i}} s_{j} \geq 2$. To this aim, suppose a strategy profile where $\sum_{j \in N_{i}} s_{j} \geq 2$. Then, the best response of player $i$ is $s_{i}=1$, since $\pi_{i}\left(0, s_{-i}\right)=50$ and $\pi_{i}\left(1, s_{-i}\right) \geq 66.66$. Suppose now a strategy profile where $s_{i}=1$ is a best response and $\sum_{j \in N_{i}} s_{j}<2$. Then, $\pi_{i}\left(0, s_{-i}\right)=50$ and $\pi_{i}\left(1, s_{-i}\right) \leq 33.33$, a contradiction. Thus, the claim follows. The claim implies that, in all Nash equilibria, $s_{i}=$ 0 if $n_{i}=1$.

Consider the Orange network. Since players $\mathrm{A}$ and $\mathrm{E}$ choose 0 in all Nash equilibria $\left(n_{A}=n_{E}=\right.$ 1 ), in a pure-strategy Nash equilibrium either $s_{B}=s_{C}=s_{D}=1$ or $s_{B}=s_{C}=s_{D}=0$. Consider 
the Green and the Purple Network. Players $A$ and $E$ in the Green network, and players $A, C$ and $E$ in the Purple network choose 0 in all Nash equilibria (all of them have $n_{i}=1$ ). Hence, in a Nash equilibrium, players B and D in the Green network and player D in the Purple network also choose action 0 , since they have $n_{i}=2$ and one of their neighbors has $n_{i}=1$ (and, therefore, chooses action 0). It follows that, in a Nash equilibrium, also player $\mathrm{C}$ in the Green network and player B in the Purple network choose action 0, since all their neighbors also choose 0 . It is straightforward to see that all the equilibria are strict. QED

\section{Incomplete information, 5-player networks}

In Incomplete information scenario players are not informed about which network has been drawn, but they know their own degree (the number of neighbors they have, either 1,2 or 3 ). With this information in hand, each player decides whether to be active (action 1) or not (action 0 ). Since each player only learns her degree (and the prior $p$ ), she can only condition her behavior on this information. In this sense, a (symmetric) strategy profile is represented by a vector $s=\left(s_{1}, s_{2}, s_{3}\right)$, where $s_{j} \in\{0,1\}$ is the action chosen by an agent with degree $j \in$ $\{1,2,3\}$. There are 8 strategy profile candidates to be a pure - strategy Nash equilibrium: $s^{\mathrm{I}}=$ $(0,0,0), s^{\mathrm{II}}=(1,0,0), s^{\mathrm{III}}=(0,1,0), s^{\mathrm{IV}}=(0,0,1), s^{\mathrm{V}}=(1,1,0), s^{\mathrm{VI}}=(1,0,1), s^{\mathrm{VII}}=(0,1,1)$ and $s^{\mathrm{VIII}}=$ $(1,1,1)$. Let $\pi_{i}^{j}\left(x_{i}, x_{-i}\right)$ be the payoff of an agent (indexed by $i \in N$ ) with degree $j \in\{1,2,3\}$.

Proposition 3. In the scenario of strategic substitutes and incomplete information of Experiment 1 there exists a unique pure-strategy Bayes-Nash equilibrium: $\left(1, s_{2}^{*}, 0\right)$, with $s_{2}^{*}=0$ if $p=0.2$ and $s_{2}^{*}=1$ if $p=0.8$. All these equilibria are strict.

Proof. We first define some conditional probabilities that shall be useful in the proof. Let $q_{1}(j)$ be the expected probability for an agent that, conditional on having degree 1, her neighbor has degree $j$. By applying Bayes' rule we get $q_{1}(2)=\frac{3(1-p)}{5(1-p)+4 p}$ and $q_{1}(3)=\frac{2(1-p)+4 p}{5(1-p)+4 p}$. Let $q_{2}\left(j_{1} j_{2}\right)$ be the expected probability for an agent that, conditional on having degree 2 , her neighbors have degrees $j_{1}$ and $j_{2}$. By applying Bayes' rule we get $q_{2}(1,2)=\frac{2(1-p)}{4(1-p)+2 p}, q_{2}(2,2)=\frac{1-p}{4(1-p)+2 p}, q_{2}(1,3)$ $=\frac{1-p}{4(1-p)+2 p}$ and $q_{2}(3,3)=\frac{2 p}{4(1-p)+2 p}$. Let $q_{3}\left(j_{1}, j_{2}, j_{3}\right)$ be the expected probability for an agent that, conditional on having degree 3 , her neighbors have degrees $j_{1}, j_{2}$ and $j_{3}$. By applying Bayes' rule we get $q_{3}(1,1,2)=\frac{1-p}{1+3 p}$ and $q_{3}(1,2,3)=\frac{4 p}{1+3 p}$.

First, we prove that candidates $s^{\mathrm{I}}, s^{\mathrm{III}}, s^{\mathrm{IV}}, s^{\mathrm{VI}}, s^{\mathrm{VII}}$ and $s^{\mathrm{VIII}}$ cannot be equilibria.

For all $p \in(0,1), s^{\mathrm{I}}$ is not an equilibrium, since $\pi_{i}^{1}\left(0, x_{-i}^{I}\right)=0<50=\pi_{i}^{1}\left(1, x_{-i}^{I}\right)$.

Regarding $s^{I I I}$, in order to be an equilibrium, it would require $\pi_{i}^{1}\left(0, x_{-i}^{I I I}\right) \geq \pi_{i}^{1}\left(1, x_{-i}^{I I I}\right)$ and $\pi_{i}^{2}\left(1, x_{-i}^{I I I}\right) \geq \pi_{i}^{2}\left(0, x_{-i}^{I I I}\right)$, i.e., $q_{1}(2) \geq \frac{1}{2}$ and $\frac{1}{2} \geq q_{2}(1,2)+q_{2}(2,2)$, but these inequalities are incompatible for all $p \in(0,1)$. 
Regarding $s^{\mathrm{IV}}$, in order to be an equilibrium, it would require $\pi_{i}^{2}\left(0, x_{-i}^{I V}\right) \geq \pi_{i}^{2}\left(1, x_{-i}^{I V}\right)$ and $\pi_{i}^{3}\left(1, x_{-i}^{I V}\right) \geq \pi_{i}^{3}\left(0, x_{-i}^{I V}\right)$, i.e., $q_{2}(1,3)+q_{2}(3,3) \geq \frac{1}{2}$ and $\frac{1}{2} \geq q_{3}(1,2,3)$, but these inequalities are incompatible for all $p \in(0,1)$.

For all $p \in(0,1), s^{\mathrm{VI}}$ is not an equilibrium, since $\pi_{i}^{3}\left(1, x_{-i}^{V I}\right)=50<100=\pi_{i}^{3}\left(0, x_{-i}^{V I}\right)$.

For all $p \in(0,1), s^{\mathrm{VII}}$ is not an equilibrium, since $\pi_{i}^{2}\left(1, x_{-i}^{V I I}\right)=50<100=\pi_{i}^{2}\left(0, x_{-i}^{V I I}\right)$.

For all $p \in(0,1), s^{\mathrm{VIII}}$ is not an equilibrium, since $\pi_{i}^{1}\left(1, x_{-i}^{V I I I}\right)=50<100=\pi_{i}^{1}\left(0, x_{-i}^{V I I I}\right)$.

Finally, we prove that candidates $s^{\mathrm{II}}$ is an equilibrium if and only if $p \leq \frac{1}{2}$, and that $s^{\mathrm{V}}$ is an equilibrium if and only if $p \geq \frac{2}{3}$. Let us start with $s^{\mathrm{II}}$. First, we observe that, for all $p \in(0,1)$, $\pi_{i}^{1}\left(1, x_{-i}^{I I}\right)=50>0=\pi_{i}^{1}\left(0, x_{-i}^{I I}\right)$ and $\pi_{i}^{3}\left(0, x_{-i}^{I I}\right)=100>50=\pi_{i}^{3}\left(1, x_{-i}^{I I}\right)$. Hence, in order to be an equilibrium, it requires $\pi_{i}^{2}\left(0, x_{-i}^{I I}\right) \geq \pi_{i}^{2}\left(1, x_{-i}^{I I}\right)$, i.e., $q_{2}(1,2)+q_{2}(1,3) \geq \frac{1}{2}$, which simplifies to $p \leq \frac{1}{2}$. Thus, if $p=0.2$, $s^{\mathrm{II}}$ is a strict equilibrium and, if $p=0.8$, it is not an equilibrium. Consider now $s^{\mathrm{V}}$. First, we observe that, for all $p \in(0,1), \pi_{i}^{3}\left(0, x_{-i}^{V}\right)=100>$ $50=\pi_{i}^{3}\left(1, x_{-i}^{V}\right)$. Hence, in order to be an equilibrium, it requires both $\pi_{i}^{1}\left(1, x_{-i}^{V}\right) \geq \pi_{i}^{1}\left(0, x_{-i}^{V}\right)$ and $\pi_{i}^{2}\left(1, x_{-i}^{V}\right) \geq \pi_{i}^{2}\left(0, x_{-i}^{V}\right)$, i.e., $\frac{1}{2} \geq q_{1}(2)$ and $\frac{1}{2} \geq q_{2}(1,2)+q_{2}(2,2)+q_{2}(1,3)$. The second inequality implies the first one, and the equilibrium condition simplifies to $p \geq \frac{2}{3}$. Thus, if $p=$ $0.2, s^{\mathrm{V}}$ is not an equilibrium and, if $p=0.8$, it is a strict equilibrium. QED

Proposition 4. In the scenario of strategic complements and incomplete information of Experiment 1, if $p=0.2$ there is a unique Bayes-Nash equilibrium: $(0,0,0)$; if $p \in\{0.8,0.95\}$, there are two pure-strategy Bayes Nash equilibria: $(0,0,0)$ and $(0,1,1)$. All these equilibria are strict.

Proof. The conditional probabilities $q_{1}(j), q_{2}\left(j_{1}, j_{2}\right)$ and $q_{3}\left(j_{1}, j_{2}, j_{3}\right)$ are defined in the proof of Proposition 3. We first prove that candidates $s^{\mathrm{II}}, s^{\mathrm{III}}, s^{\mathrm{IV}}, s^{\mathrm{V}}, s^{\mathrm{VI}}$, and $s^{\mathrm{VIII}}$ cannot be equilibria:

For all $p \in(0,1), s^{\mathrm{II}}, s^{\mathrm{V}}, s^{\mathrm{VI}}$ and $s^{\mathrm{VIII}}$ are not equilibria, since $\pi_{i}^{1}\left(1, x_{-i}^{I I}\right) \leq \frac{100}{3}<50=\pi_{i}^{1}\left(0, x_{-i}^{I I}\right)$. For all $p \in(0,1), s^{\mathrm{III}}$ is not an equilibrium, since $\pi_{i}^{2}\left(1, x_{-i}^{I I I}\right)=\frac{100}{3}\left(q_{2}(1,2)+2\left(q_{2}(2,2)\right)<\right.$ $50=\pi_{i}^{2}\left(0, x_{-i}^{I I I}\right)$. Regarding $s^{\mathrm{IV}}$, in order to be an equilibrium, it would require $\pi_{i}^{3}\left(1, x_{-i}^{I V}\right) \geq$ $\pi_{i}^{3}\left(0, x_{-i}^{I V}\right)$, i.e., $\frac{100}{3} q_{3}(1,2,3) \geq 50$. However, the inequality does not hold since, for any $\in(0,1)$, $q_{3}(1,2,3)<1$.

We now prove that candidates $s^{\mathrm{I}}$ is an equilibrium for all $p \in(0,1)$, and that candidate $s^{\mathrm{VII}}$ is an equilibrium if and only if $p \geq 1 / 2$. We start with candidate $s^{\mathrm{I}}$. For all $p \in(0,1)$, and $k \in\{1,2,3\}$, $\pi_{i}^{k}\left(0, x_{-i}\right)=50>0=\pi_{i}^{k}\left(1, x_{-i}\right)$. Hence $s^{\mathrm{I}}$ is a strict equilibrium. Now consider candidate $s^{\mathrm{VII}}$. First, we observe that, for all $p \in(0,1), \pi_{i}^{1}\left(0, x_{-i}^{V I I}\right)=50>100 / 3=\pi_{i}^{1}\left(1, x_{-i}^{V I I}\right)$. Hence, in order to be an equilibrium, it requires both $\pi_{i}^{2}\left(1, x_{-i}^{V I I}\right) \geq \pi_{i}^{2}\left(0, x_{-i}^{V I I}\right)$ and $\pi_{i}^{2}\left(1, x_{-i}^{V I I}\right) \geq$ $\pi_{i}^{2}\left(0, x_{-i}^{V I I}\right)$, i.e., 


$$
\begin{aligned}
& \frac{100}{3}\left(q_{2}(1,2)+q_{2}(1,3)+2 q_{2}(2,2)+2 q_{2}(2,3)\right) \geq 50 \text { and } \\
& \frac{100}{3}\left(q_{3}(1,1,2)+2 q_{3}(1,2,3)\right) \geq 50 .
\end{aligned}
$$

The first inequality simplifies to $p \geq 1 / 2$ and the second one simplifies to $p \geq 1 / 5$. Hence, if $p=$ $0.2, s^{\mathrm{VII}}$ is not an equilibrium and, if $p=0.8$ it is a strict equilibrium (since both inequalities strictly hold). QED

Proposition 5. In the scenario of Experiment 2 (strategic complements and incomplete information), (i) if $p=0.2$, there are two pure-strategy Bayes-Nash equilibria: $(0,0,0)$ and $(0,1,1)$; these equilibria are strict. (ii) If $p=0.8$ there are two pure-strategy strict Bayes-Nash equilibria: $(0,0,0)$ and $(0,1,1)$; and there is a pure-strategy weak Bayes-Nash equilibria: $(0,0,1)$.

Proof. We first redefine the conditional probabilities for the case of Experiment 2. By applying Bayes' rule we get $q_{1}(2)=\frac{1-p}{2}, q_{1}(3)=\frac{1+p}{2}, q_{2}(2,2)=\frac{1-p}{2(p+3(1-p))^{\prime}}, q_{2}(1,3)=\frac{1-p}{2(p+3(1-p))^{\prime}}, q_{2}(2,3)=$ $\frac{2(1-p)}{p+3(1-p)}, q_{2}(3,3)=\frac{p}{p+3(1-p)}, q_{3}(1,2,2)=\frac{1-p}{2(3 p+(1-p))}, q_{3}(2,2,2)=\frac{1-p}{2(3 p+(1-p))}, q_{3}(1,3,3)=\frac{p}{3 p+(1-p)}$, and $q_{3}(2,3,3)=\frac{2 p}{3 p+(1-p)}$.

For all $p \in(0,1), s^{\mathrm{II}}, s^{\mathrm{V}}, s^{\mathrm{VI}}$ and $s^{\mathrm{VIII}}$ are not equilibria, since $\pi_{i}^{1}\left(1, x_{-i}\right) \leq \frac{100}{3}<50=$ $\pi_{i}^{1}\left(0, x_{-i}\right)$. For all $p \in(0,1)$, since $\pi_{i}^{2}\left(1, x_{-i}^{I I I}\right)=\frac{100}{3}\left(2 q_{2}(2,2)+q_{2}(2,3)\right)<50=\pi_{i}^{2}\left(0, x_{-i}^{I I I}\right)$, $s^{\mathrm{III}}$ is not an equilibrium.

We now prove that candidate $s^{\mathrm{I}}$ is an equilibrium for all $p \in(0,1)$, and that candidate $s^{\mathrm{VII}}$ is an equilibrium if and only if $p \geq 1 / 2$. We start with candidate $s^{\mathrm{I}}$. For all $p \in(0,1)$, and $k \in\{1,2,3\}$, $\pi_{i}^{k}\left(0, x_{-i}\right)=50>0=\pi_{i}^{k}\left(1, x_{-i}\right)$. Hence $s^{\mathrm{I}}$ is a strict equilibrium. Now consider candidate $s^{\mathrm{VII}}$. First, we observe that, for all $p \in(0,1), \pi_{i}^{1}\left(0, x_{-i}^{V I I}\right)=50>100 / 3=\pi_{i}^{1}\left(1, x_{-i}^{V I I}\right)$. Hence, in order to be an equilibrium, it requires both $\pi_{i}^{2}\left(1, x_{-i}^{V I I}\right) \geq \pi_{i}^{2}\left(0, x_{-i}^{V I I}\right)$ and $\pi_{i}^{3}\left(1, x_{-i}^{V I I}\right) \geq$ $\pi_{i}^{3}\left(0, x_{-i}^{V I I}\right)$, i.e.,

$$
\begin{aligned}
& \frac{100}{3}\left(2 q_{2}(2,2)+q_{2}(1,3)+2 q_{2}(2,3)+2 q_{2}(3,3)\right) \geq 50 \text { and } \\
& \frac{100}{3}\left(2 q_{3}(1,2,2)+3 q_{3}(2,2,2)+2 q_{3}(1,3,3)+3 q_{3}(2,3,3)\right) \geq 50 .
\end{aligned}
$$

It can be directly verified that both inequalities (strictly) hold for any $p \in(0,1)$ and, therefore, $s^{\mathrm{VII}}$ is a strict equilibrium.

Finally, consider $s^{\mathrm{IV}}$. First, we observe that, for all $p \in(0,1), \pi_{i}^{1}\left(0, x_{-i}^{I V}\right)=50>100 / 3=$ $\pi_{i}^{1}\left(1, x_{-i}^{I V}\right)$. Hence, in order to be an equilibrium, it requires both $\pi_{i}^{2}\left(0, x_{-i}^{I V}\right) \geq \pi_{i}^{2}\left(1, x_{-i}^{I V}\right)$ and $\pi_{i}^{3}\left(1, x_{-i}^{I V}\right) \geq \pi_{i}^{3}\left(0, x_{-i}^{I V}\right)$, i.e.,

$$
\begin{aligned}
& 50 \geq \frac{100}{3}\left(q_{2}(1,3)+q_{2}(2,3)+2 q_{2}(3,3)\right) \text { and } \\
& \frac{100}{3}\left(2 q_{3}(1,3,3)+2 q_{3}(2,3,3)\right) \geq 50 .
\end{aligned}
$$

The first inequality simplifies to $p \leq 0.8$ and the second one simplifies to $p \geq 0.5$. Therefore, $s^{\mathrm{IV}}$ is not an equilibrium if $p=0.2$, and it is a weak equilibrium if $p=0.8$. QED 


\section{Incomplete information, 20-player networks}

In this scenario players are informed about which network is in place but they do not know the specific position they have in the network. They know only their own degree (the number of neighbors they have, either 1, 2, 3 or 4). With this information in hand, each player decides whether to be active (action 1) or not (action 0). Since each player only learns her degree, she can only condition her behavior on this information. In this sense, a (symmetric) strategy profile is represented by a vector $s=\left(s_{1}, s_{2}, s_{3}, s_{4}\right)$, where $s_{j} \in\{0,1\}$ is the action chosen by an agent with degree $\mathrm{j} \in\{1,2,3,4\}$.

Proposition 6. In the scenario of Experiment 3 in the three 20-player networks there are two pure-strategy Bayes-Nash equilibria: $(0,0,0,0)$ and $(0,1,1,1)$. Both equilibria are strict.

Proof. Let $\pi_{i}^{j}\left(x_{i}, x_{-i}\right) \equiv \pi_{i}^{j}\left(x_{i}\right)$ be the payoff of an agent (indexed by $i \in N$ ) with degree $j \in$ $\{1,2,3,4\}$ from action $x_{i}$ when other players choose $x_{-i}$. Note that (i) in all Nash equilibria, $s_{1}=0$ because $\pi_{i}^{1}\left(0, x_{-i}\right)=50$ and $\pi_{i}^{1}\left(x_{i}, x_{-i}\right) \leq \frac{100}{3}$; (ii) the strategy profile $(0,0,0,0)$ is a strict Bayes-Nash equilibrium in all networks because a deviation to action 1 produces a payoff of 0 (against a payoff of 50 from action 0 ). Then there are 7 strategy profiles candidates to be a purestrategy Bayes-Nash equilibrium: all possible combinations of $s_{2}, s_{3}, s_{4}$ in $s$ excluding $(0,0,0,0)$.

Consider Network 1. Strategy $(0,1,0,0)$ is not an equilibrium because $\pi_{i}^{2}\left(1, x_{-i}\right)=$ $22.22 \leq 50=\pi_{i}^{2}\left(0, x_{-i}\right)$. Strategy $(0,0,1,0)$ is not an equilibrium because $\pi_{i}^{3}\left(1, x_{-i}\right)=16.66<$ $50=\pi_{i}^{3}\left(0, x_{-i}\right)$. Strategy $(0,0,0,1)$ is not an equilibrium because $\pi_{i}^{4}\left(1, x_{-i}\right)=0<50=$ $\pi_{i}^{4}\left(0, x_{-i}\right)$. Strategy $(0,1,1,0)$ is not an equilibrium because $\pi_{i}^{4}\left(1, x_{-i}\right)=116.66>50=$ $\pi_{i}^{4}\left(0, x_{-i}\right)$. Strategy $(0,1,0,1)$ is not an equilibrium because $\pi_{i}^{2}\left(1, x_{-i}\right)=44.44<50=$ $\pi_{i}^{2}\left(0, x_{-i}\right)$. Strategy $(0,0,1,1)$ is not an equilibrium because $\pi_{i}^{3}\left(1, x_{-i}\right)=41.66<50=$ $\pi_{i}^{3}\left(0, x_{-i}\right)$. Finally, strategy $(0,1,1,1)$ is a strict equilibrium because $\pi_{i}^{4}\left(1, x_{-i}\right)=116.66>50=$ $\pi_{i}^{4}\left(0, x_{-i}\right), \pi_{i}^{3}\left(1, x_{-i}\right)=58.33>50=\pi_{i}^{3}\left(0, x_{-i}\right)$, and $\pi_{i}^{2}\left(1, x_{-i}\right)=55.55>50=\pi_{i}^{2}\left(0, x_{-i}\right)$.

Consider Network 2. Strategy $(0,1,0,0)$ is not an equilibrium because $\pi_{i}^{2}\left(1, x_{-i}\right)=0 \leq$ $50=\pi_{i}^{2}\left(0, x_{-i}\right)$. Strategy $(0,0,1,0)$ is not an equilibrium because $\pi_{i}^{3}\left(1, x_{-i}\right)=11.11<50=$ $\pi_{i}^{3}\left(0, x_{-i}\right)$. Strategy $(0,0,0,1)$ is not an equilibrium because $\pi_{i}^{4}\left(1, x_{-i}\right)=44.44<50=$ $\pi_{i}^{4}\left(0, x_{-i}\right)$. Strategy $(0,1,1,0)$ is not an equilibrium because $\pi_{i}^{2}\left(1, x_{-i}\right)=41.66<50=$ $\pi_{i}^{2}\left(0, x_{-i}\right)$. Strategy $(0,1,0,1)$ is not equilibrium because $\pi_{i}^{2}\left(1, x_{-i}\right)=25<50=\pi_{i}^{2}\left(0, x_{-i}\right)$. Strategy $(0,0,1,1)$ is not equilibrium because $\pi_{i}^{2}\left(1, x_{-i}\right)=66.66>50=\pi_{i}^{2}\left(0, x_{-i}\right)$. Strategy $(0,1,1,1)$ is a strict equilibrium because $\pi_{i}^{4}\left(1, x_{-i}\right)=116.66>50=\pi_{i}^{4}\left(0, x_{-i}\right), \pi_{i}^{3}\left(1, x_{-i}\right)=$ $94.44>50=\pi_{i}^{3}\left(0, x_{-i}\right)$, and $\pi_{i}^{2}\left(1, x_{-i}\right)=66.66>50=\pi_{i}^{2}\left(0, x_{-i}\right)$.

Consider Network 3. Strategy $(0,1,0,0)$ is not an equilibrium because $\pi_{i}^{2}\left(1, x_{-i}\right)=0 \leq$ $50=\pi_{i}^{2}\left(0, x_{-i}\right)$. Strategy $(0,0,1,0)$ is not an equilibrium because $\pi_{i}^{3}\left(1, x_{-i}\right)=11.11<50=$ 
$\pi_{i}^{3}\left(0, x_{-i}\right)$. Strategy $(0,0,0,1)$ is not an equilibrium because $\pi_{i}^{4}\left(1, x_{-i}\right)=33.33<50=$ $\pi_{i}^{4}\left(0, x_{-i}\right)$. Strategy $(0,1,1,0)$ is not an equilibrium because $\pi_{i}^{2}\left(1, x_{-i}\right)=33.33<50=$ $\pi_{i}^{2}\left(0, x_{-i}\right)$. Strategy $(0,1,0,1)$ is not an equilibrium because $\pi_{i}^{2}\left(1, x_{-i}\right)=33.33<50=$ $\pi_{i}^{2}\left(0, x_{-i}\right)$. Strategy $(0,0,1,1)$ is not an equilibrium because $\pi_{i}^{2}\left(1, x_{-i}\right)=66.66>50=$ $\pi_{i}^{2}\left(0, x_{-i}\right)$. Strategy $(0,1,1,1)$ is a strict equilibrium because $\pi_{i}^{4}\left(1, x_{-i}\right)=116.66>50=$ $\pi_{i}^{4}\left(0, x_{-i}\right), \pi_{i}^{3}\left(1, x_{-i}\right)=94.44>50=\pi_{i}^{3}\left(0, x_{-i}\right)$, and $\pi_{i}^{2}\left(1, x_{-i}\right)=66.66>50=\pi_{i}^{2}\left(0, x_{-i}\right)$. QED 


\section{Online Supplement A: Figures by groups}

$(\mathrm{x}, \mathrm{y}, \mathrm{z})$ means group $\mathrm{x}$, network y position $\mathrm{z}$ where Network: Orange $=1$, Green $=2$, Purple $=3$ Position: $A=1, B=2, C=3, D=4, E=5$.

Figure B.1: Complete information and substitutes: Relative frequencies of active choices across periods, by group, network and position.

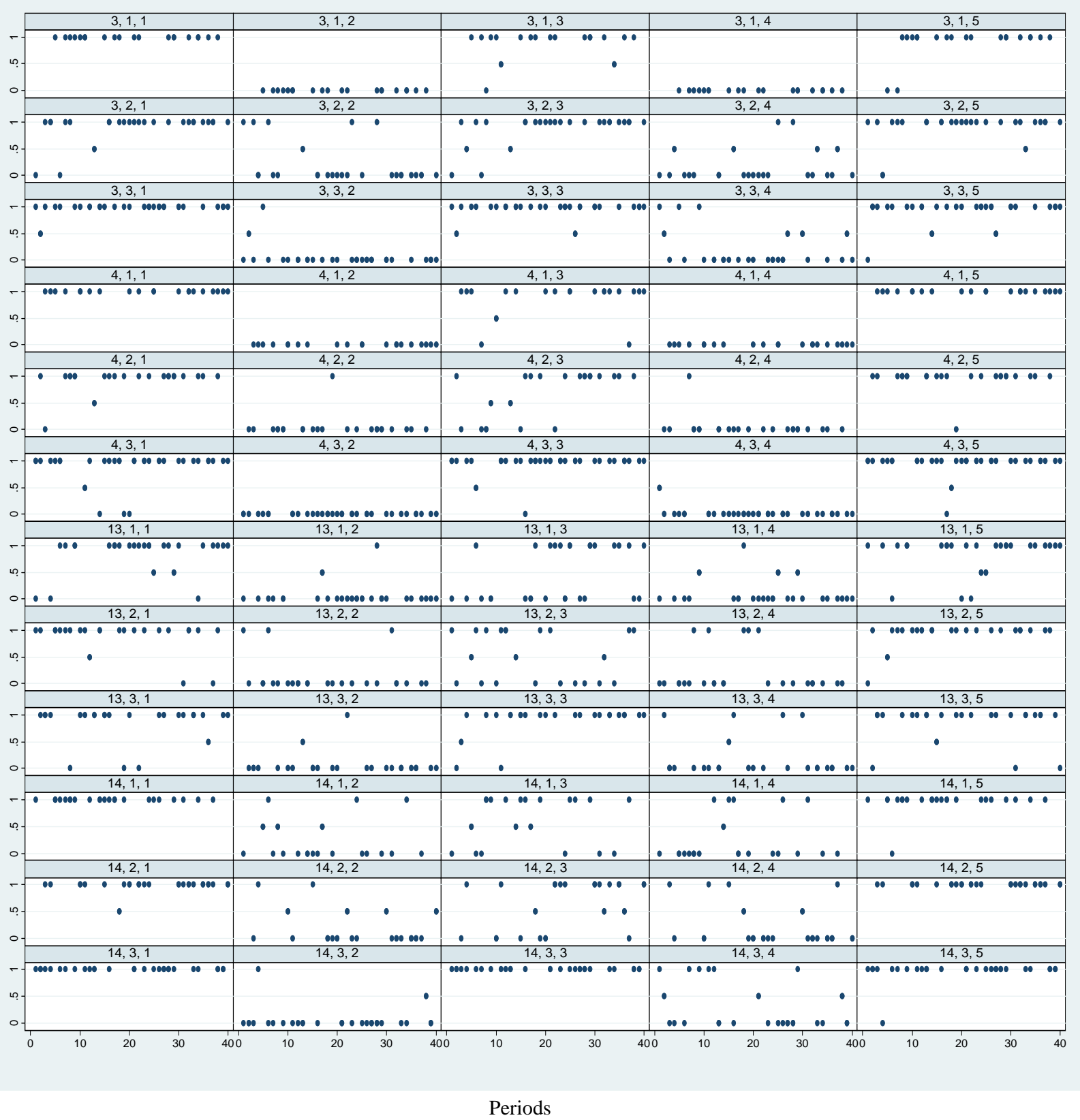


Figure B.2: Complete information and complements: Relative frequencies of active choices across periods, by group and position in the Orange network.

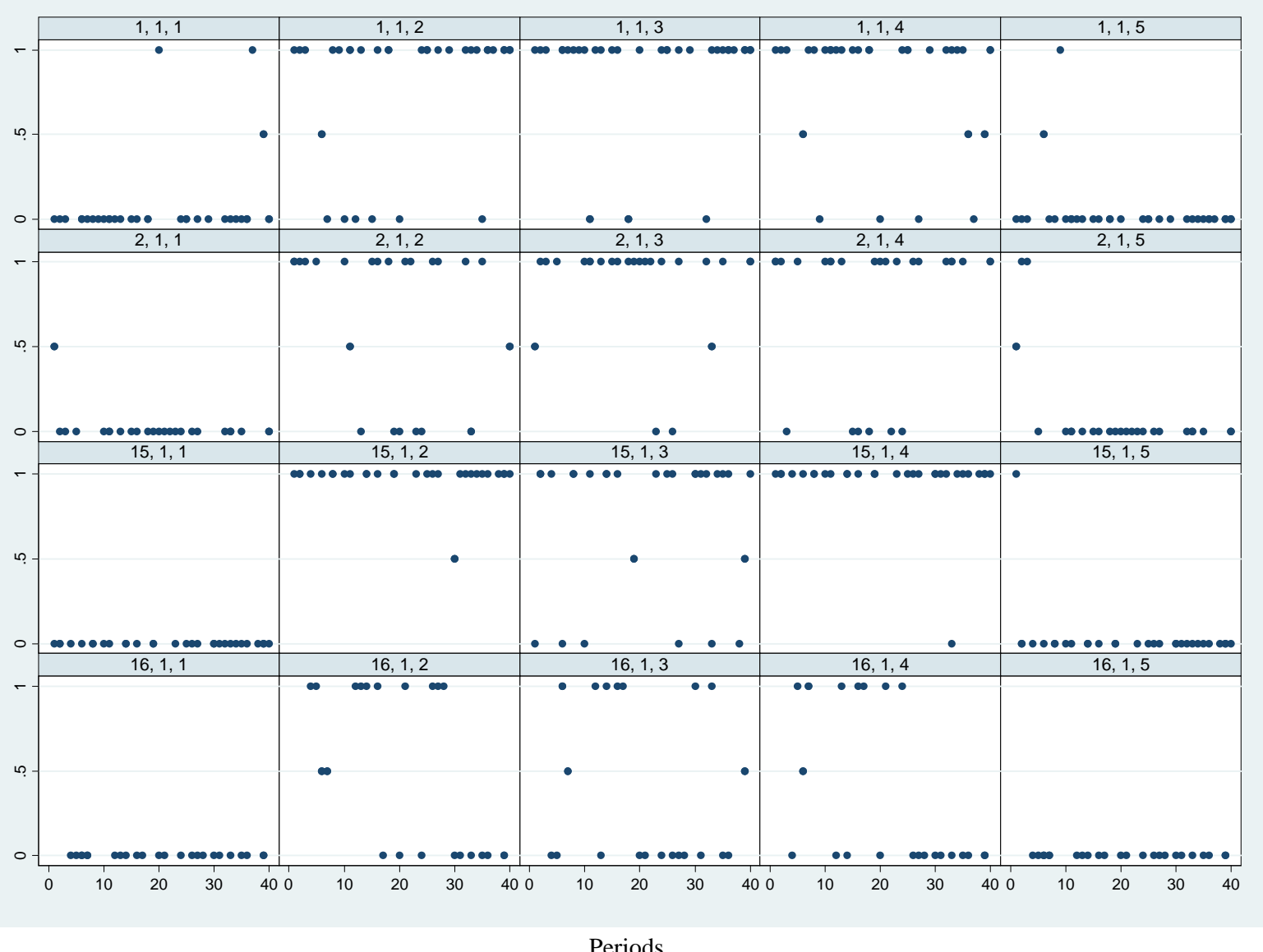

Periods 


\section{Online Supplement B: Econometric model (Variables and Estimations)}

\section{Experiments 1 and 2}

\section{Network:}

Experiment 1

Orange $=1$,

Green $=2$,

Purple $=3$

Experiment 2

Blue $=1$,

Red $=2$,

Brown $=3$

\section{Position:}

$$
\begin{aligned}
& A=1, \\
& B=2, \\
& C=3, \\
& D=4, \\
& E=5 .
\end{aligned}
$$

\section{Complete information}

dij $=1$ if network $=\mathrm{i}$ and position $=\mathrm{j}, 0$ otherwise tij: interaction between dij and period

\section{Incomplete information}

$\mathrm{d} 1=1$ if $\mathrm{p}=0.8,0$ otherwise degree $2=1$ if player's degree $=2,0$ otherwise degree $3=1$ if player's degree $=3$, 0 otherwise d1_period: interaction between period and d1 d1_degree2: interaction between $\mathrm{d} 1$ and degree2 d1_degree3: interaction between $\mathrm{d} 1$ and degree 3 deg2_period: interaction between degree2 and period deg3_period: interaction between degree3 and period deg2_per_d1: interaction between degree2, period and d1 deg3_per_d1: interaction between degree3, period and d1.

risk_0_1: marginal effect of risk when $\mathrm{d} 1=0$ and degree $==1$ risk_0_2: marginal effect of risk when $\mathrm{d} 1=0$ and degree $==2$ risk_0_3: marginal effect of risk when $\mathrm{d} 1=0$ and degree $==3$ risk_1_1: marginal effect of risk when $\mathrm{d} 1=1$ and degree $==1$ risk_1_2: marginal effect of risk when $\mathrm{d} 1=1$ and degree $==2$ risk_1_3: marginal effect of risk when $\mathrm{d} 1=1$ and degree $==3$ 


\section{Experiment 1}

\section{Complete information - Strategic Substitutes}

\begin{tabular}{|c|c|c|c|c|c|c|}
\hline Choice & Coef. & Std. Err. & $\mathrm{z}$ & $\mathrm{P}>\mathrm{Z}$ & \multicolumn{2}{|c|}{ [95\% Conf.Interval] } \\
\hline Period & 0.030663 & 0.047141 & 0.65 & 0.515 & -0.06173 & 0.123057 \\
\hline d12 & -8.40485 & 1.7711 & -4.75 & $\odot$ & -11.8761 & -4.93356 \\
\hline d13 & -3.90144 & 1.265561 & -3.08 & $\odot .0 \odot 2$ & -6.38189 & -1.42098 \\
\hline $\mathrm{d} 14$ & -6.95259 & 1.5288 & -4.55 & 0 & -9.94899 & -3.9562 \\
\hline d15 & -1.80211 & 1.416295 & -1.27 & 0.203 & -4.578 & 0.973777 \\
\hline $\mathrm{d} 21$ & -2.43685 & 1.350567 & -1.8 & 0.071 & -5.08391 & 0.210213 \\
\hline $\mathrm{d} 22$ & -5.75513 & 1.357171 & -4.24 & $\odot$ & -8.41514 & -3.09512 \\
\hline d23 & -4.33103 & 1.260564 & -3.44 & 0.001 & -6.80169 & -1.86037 \\
\hline $\mathrm{d} 24$ & -5.59522 & 1.326514 & -4.22 & $\odot$ & -8.19514 & -2.9953 \\
\hline d25 & -1.22466 & 1.440592 & -0.85 & 0.395 & -4.04816 & 1.598853 \\
\hline d31 & $-\odot .87704$ & 1.366207 & -0.64 & 0.521 & -3.55476 & 1.800676 \\
\hline d32 & -6.18386 & 1.418038 & -4.36 & 0 & -8.96316 & -3.40455 \\
\hline d33 & -2.10023 & 1.34226 & -1.56 & 0.118 & -4.73101 & 0.530551 \\
\hline d34 & -4.85752 & 1.249787 & -3.89 & $\odot$ & -7.30706 & -2.40798 \\
\hline d35 & -1.86108 & 1.328373 & -1.4 & 0.161 & -4.46464 & 0.742488 \\
\hline $\mathrm{t} 12$ & -0.03139 & ๑. 073418 & -0.43 & 0.669 & -0.17528 & 0.112509 \\
\hline $\mathrm{t} 13$ & 0.02826 & $\odot .053593$ & 0.53 & $\odot .598$ & -0.07678 & 0.133299 \\
\hline $\mathrm{t} 14$ & $-\odot .06707$ & $\odot .065325$ & -1.03 & 0.305 & $-\odot .19511$ & $\odot .060959$ \\
\hline $\mathrm{t} 15$ & $\odot .053105$ & 0.068362 & 0.78 & 0.437 & -0.08088 & 0.187091 \\
\hline $\mathrm{t} 21$ & $\odot .078902$ & ๑. 061831 & 1.28 & 0.202 & -0.04228 & 0.200088 \\
\hline $\mathrm{t} 22$ & $-\odot .0878$ & $\odot .059385$ & -1.48 & $\odot .139$ & $-\odot .20419$ & 0.028597 \\
\hline $\mathrm{t} 23$ & $\odot .046098$ & $\odot .053475$ & 0.86 & 0.389 & -0.05871 & 0.150906 \\
\hline $\mathrm{t} 24$ & -0.0617 & $\odot .05645$ & -1.09 & 0.274 & -0.17234 & 0.048942 \\
\hline $\mathrm{t} 25$ & 0.050011 & $\odot .074264$ & 0.67 & 0.501 & -0.09554 & 0.195565 \\
\hline t31 & 0.005485 & ๑. 061208 & $\odot . \odot 9$ & $\odot .929$ & -0.11448 & 0.125452 \\
\hline$t 32$ & $-\odot .13539$ & $\odot .06704$ & -2.02 & 0.043 & -0.26678 & -0.00399 \\
\hline t33 & 0.106867 & $\odot .072718$ & 1.47 & 0.142 & -0.03566 & ๑.249391 \\
\hline t34 & -0.09514 & $\odot .05377$ & -1.77 & $\odot .077$ & -0.20053 & 0.010246 \\
\hline t35 & $\odot .040081$ & ๑. 058256 & 0.69 & $\odot .491$ & $-\odot .0741$ & 0.154262 \\
\hline Risk & $\odot .008371$ & ๑. 011682 & 0.72 & 0.474 & -0.01453 & 0.031267 \\
\hline _cons & 3.491075 & 1.312174 & 2.66 & $\odot .0 \odot 8$ & ๑. 919263 & 6.062888 \\
\hline
\end{tabular}

Marginal effects of risk

\begin{tabular}{rrrrrrr}
\hline & Coef. & Std. Err. & $\mathrm{z}$ & $\mathrm{P}>\mathrm{z}$ & {$[95 \%$ Conf. } & Interval] \\
\hline Risk & .0083711 & .01168 & 0.72 & 0.474 & -.014525 & .031267 \\
\hline
\end{tabular}




\section{Complete information - Strategic Complements}

\begin{tabular}{|c|c|c|c|c|c|c|}
\hline Choice & Coef. & Std. Err. & $z$ & $\mathrm{P}>\mathrm{Z}$ & \multicolumn{2}{|c|}{ [95\% Conf.Interval] } \\
\hline Period & 0.012517 & 0.045182 & 0.28 & 0.782 & -0.07604 & 0.101071 \\
\hline d12 & 7.203613 & 1.407627 & 5.12 & $\odot$ & 4.444714 & 9.962512 \\
\hline d13 & 5.912277 & 1.359848 & 4.35 & $\odot$ & 3.247023 & 8.57753 \\
\hline d14 & 7.21883 & 1.402497 & 5.15 & $\odot$ & 4.469987 & 9.967673 \\
\hline d15 & 4.015273 & 1.500287 & 2.68 & 0.007 & 1.074765 & 6.955781 \\
\hline d21 & 1.862151 & 2.698071 & $\odot .69$ & 0.49 & -3.42597 & 7.150273 \\
\hline d22 & 2.566745 & 1.610425 & 1.59 & 0.111 & -0.58963 & 5.723119 \\
\hline d23 & 5.055612 & 1.463772 & 3.45 & $\odot .001$ & 2.186673 & 7.924552 \\
\hline d24 & 2.257243 & 1.837471 & 1.23 & 0.219 & -1.34413 & 5.85862 \\
\hline d25 & 1.806821 & 2.147597 & 0.84 & 0.4 & -2.40239 & 6.016034 \\
\hline d31 & 4.098842 & 2.678017 & 1.53 & 0.126 & -1.14998 & 9.347658 \\
\hline d32 & 4.726608 & 1.400217 & 3.38 & $\odot .001$ & 1.982232 & 7.470983 \\
\hline d33 & 1.841959 & 2.148467 & $\odot .86$ & 0.391 & -2.36896 & 6.052878 \\
\hline d34 & 3.49502 & 1.504369 & 2.32 & 0.02 & 0.546511 & 6.443529 \\
\hline d35 & 3.505376 & 2.435331 & 1.44 & 0.15 & -1.26779 & 8.278537 \\
\hline t12 & $-\odot .04634$ & $\odot .051205$ & -0.9 & 0.365 & -0.1467 & 0.05402 \\
\hline $\mathrm{t} 13$ & -0.01217 & $\odot .05 \odot 444$ & -0.24 & 0.809 & -0.11104 & $\odot .086698$ \\
\hline t14 & -0.05783 & $\odot .050785$ & -1.14 & $\odot .255$ & -0.15737 & $\odot .041705$ \\
\hline $\mathrm{t} 15$ & -0.35341 & 0.138617 & -2.55 & 0.011 & -0.62509 & -0.08172 \\
\hline t21 & $-\odot .36076$ & $\odot .413782$ & -0.87 & 0.383 & -1.17175 & 0.450241 \\
\hline t22 & -0.1474 & ๑.088299 & -1.67 & 0.095 & -0.32047 & $\odot .025659$ \\
\hline t23 & -0.25116 & $\odot .083561$ & -3.01 & 0.003 & -0.41494 & -0.08738 \\
\hline t24 & -0.23798 & $\odot .174672$ & -1.36 & 0.173 & -0.58033 & 0.104369 \\
\hline t25 & -0.2018 & 0.16164 & -1.25 & 0.212 & -0.51861 & 0.115004 \\
\hline t31 & -0.97318 & $\odot .975$ & -1 & 0.318 & -2.88415 & $\odot .93778$ \\
\hline t32 & -0.15397 & $\odot .062666$ & -2.46 & 0.014 & -0.2768 & -0.03115 \\
\hline t33 & -0.31792 & $\odot .270881$ & -1.17 & $\odot .241$ & -0.84884 & $\odot .212999$ \\
\hline t34 & -0.20028 & ๑.090852 & -2.2 & 0.027 & -0.37835 & -0.02222 \\
\hline t35 & -0.61405 & $\odot .566495$ & -1.08 & 0.278 & -1.72436 & $\odot .496265$ \\
\hline Risk & $\odot .027471$ & $\odot . \odot \odot 7972$ & 3.45 & $\odot .001$ & 0.011846 & $\odot .043095$ \\
\hline _cons & -6.23145 & 1.347641 & -4.62 & $\odot$ & -8.87277 & -3.59012 \\
\hline
\end{tabular}

Marginal effects of risk

\begin{tabular}{rrrrrrr}
\hline & Coef. & Std. Err. & z & P>z & [95\% Conf. & Interval] \\
\hline Risk & .0274705 & .00797 & 3.45 & 0.001 & .011846 & .043095 \\
\hline
\end{tabular}


Incomplete information - Strategic Substitutes

\begin{tabular}{|c|c|c|c|c|c|c|}
\hline \multicolumn{7}{|c|}{ Log likelihood $=-835.0852$} \\
\hline Choice & Coef. & Std. Err. & $z$ & $\mathrm{P}>\mathrm{Z}$ & \multicolumn{2}{|c|}{ [95\% Conf. Interval] } \\
\hline Period & .0353746 & .0157977 & 2.24 & 0.025 & .0044117 & .0663375 \\
\hline d1 & -.6814024 & .6378184 & -1.07 & 0.285 & -1.931503 & .5686988 \\
\hline degree2 & -4.581403 & .4443026 & -10.31 & 0.000 & -5.45222 & -3.710586 \\
\hline degree3 & -7.392577 & 1.027527 & -7.19 & 0.000 & -9.406494 & -5.37866 \\
\hline d1_period & .0466369 & .0242576 & 1.92 & 0.055 & - . $๑ \odot \odot 9 \odot 71$ & .094181 \\
\hline d1_degree2 & 1.671407 & .6139628 & 2.72 & $\odot .006$ & .4680618 & 2.874752 \\
\hline d1_degree3 & 2.461545 & 1.148353 & 2.14 & $\odot . \odot 32$ & .210815 & 4.712274 \\
\hline deg2_period & - . . 0500117 & .0189244 & -2.64 & $\odot . \odot \odot 8$ & - . 0871029 & - . 0129206 \\
\hline deg3_period & -.162495 & .0831316 & -1.95 & 0.051 & -.32543 & .0004399 \\
\hline deg2_per_d1 & .0302095 & .0289505 & 1.04 & $\odot .297$ & - .0265325 & .0869514 \\
\hline deg3_per_d1 & .0148065 & .0875422 & 0.17 & $\odot .866$ & - .1567731 & .1863861 \\
\hline Risk & -.0158006 & .0069383 & -2.28 & 0.023 & - . .0293993 & - . . 0022018 \\
\hline _cons & 4.136116 & .5870075 & 7.05 & 0.000 & 2.985603 & 5.28663 \\
\hline \multicolumn{7}{|c|}{ Marginal effect of risk } \\
\hline risk_0_1 & - . .๑००2897 & .0001697 & -1.71 & 0.088 & -.0006223 & .0000429 \\
\hline risk_0_2 & - . .0021784 & .0010144 & -2.15 & $\odot .032$ & -.0041666 & - . . 0001901 \\
\hline risk_0_3 & - . .๑०००198 & .0000251 & -0.79 & $\odot .431$ & -.000069 & .0000294 \\
\hline risk_1_1 & - . .๑๑2272 & .0001254 & -1.81 & $\odot . \odot 7 \odot$ & - . .๑๑४4729 & $.00 \odot 0185$ \\
\hline risk_1_2 & - . 0032382 & .0014454 & -2.24 & $\odot . \odot 25$ & - . . 060711 & - . . 0004052 \\
\hline risk_1_3 & - . .0003819 & .0002303 & -1.66 & $\odot .097$ & - . .0008333 & .0000695 \\
\hline
\end{tabular}


Incomplete information - Strategic Complements

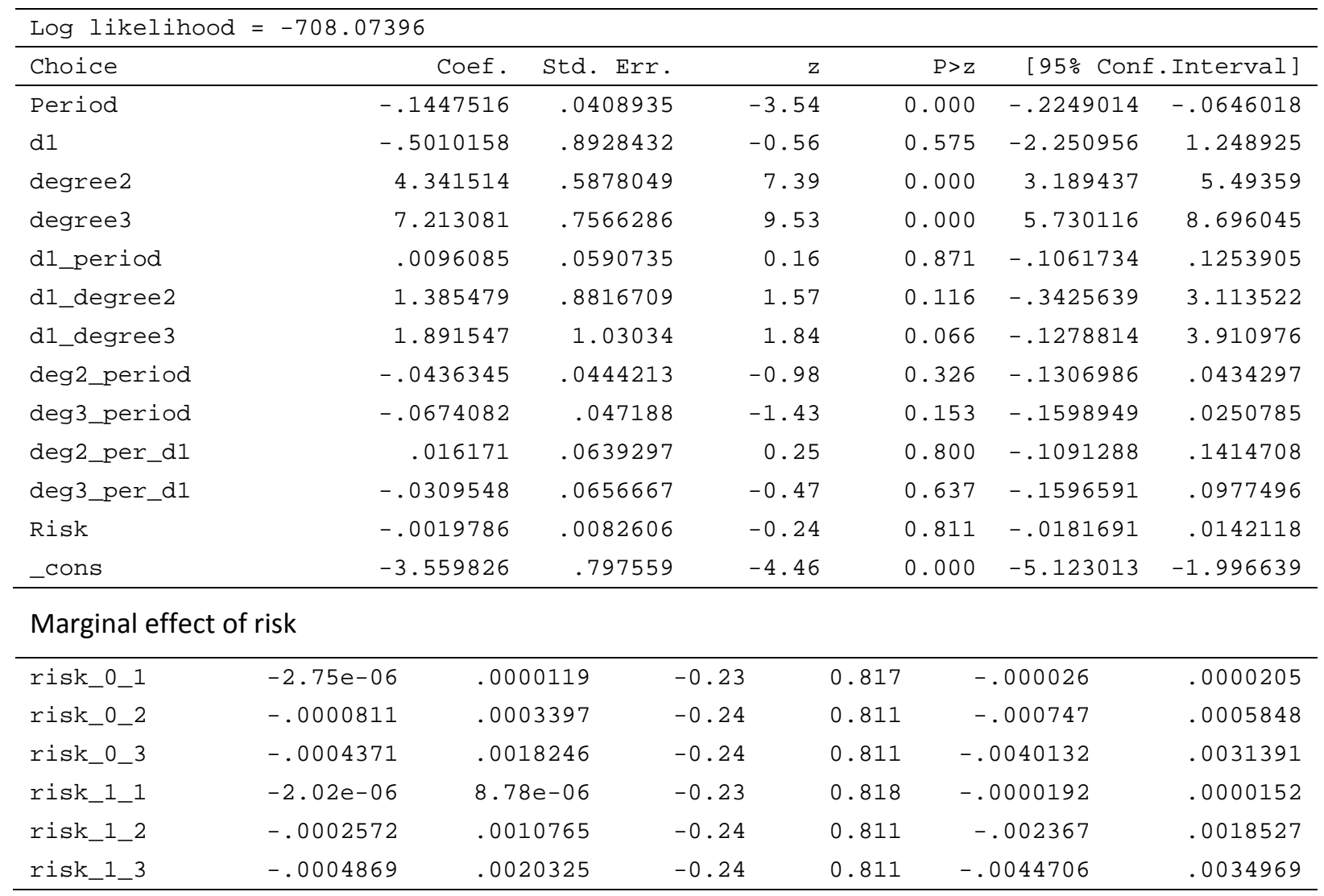

Incomplete information - Strategic Complements - $p=0.95$

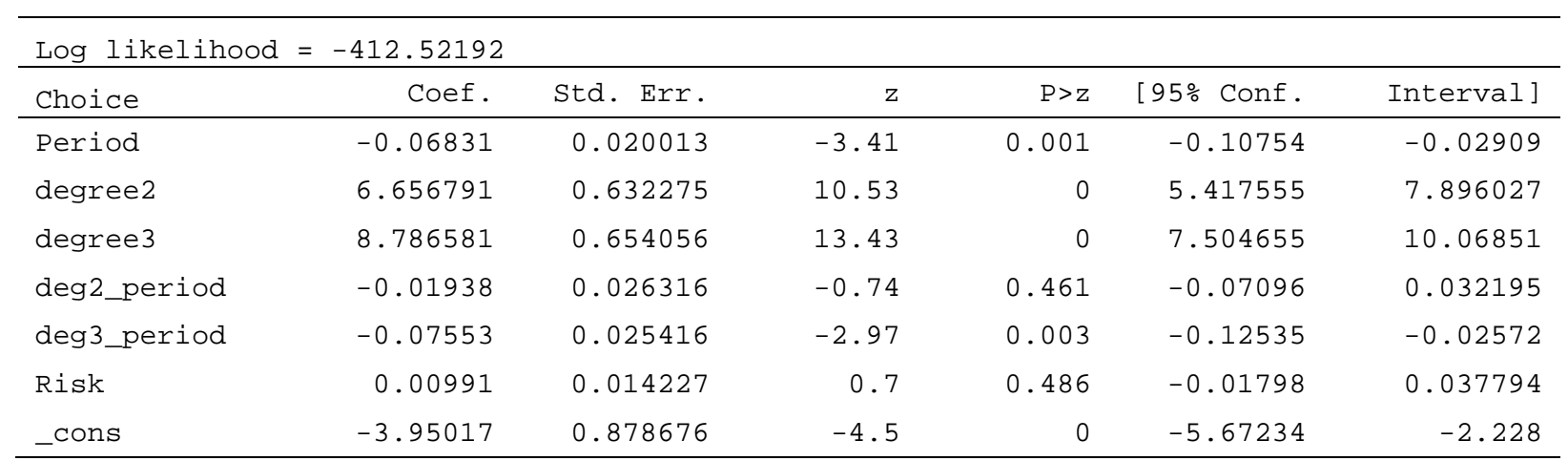




\section{Experiment 2}

\section{Complete information}

\begin{tabular}{|c|c|c|c|c|c|c|}
\hline Choice & Coef. & Std. Err. & $z$ & $\mathrm{P}>\mathrm{Z}$ & {$[95 \%$ Conf. } & Interval] \\
\hline Period & -0.78131 & 0.953394 & -0.82 & 0.412 & -2.64993 & 1.087306 \\
\hline $\mathrm{d} 212$ & 7.296092 & 2.976187 & 2.45 & $\odot .014$ & 1.462872 & 13.12931 \\
\hline $\mathrm{d} 213$ & 8.593323 & 3.199112 & 2.69 & $\odot .0 \odot 7$ & 2.323179 & 14.86347 \\
\hline d214 & 4.512357 & 2.864328 & 1.58 & 0.115 & -1.10162 & 10.12634 \\
\hline d215 & 10.06287 & 3.512437 & 2.86 & 0.004 & 3.178619 & 16.94712 \\
\hline d222 & 3.465771 & 2.897039 & 1.2 & 0.232 & -2.21232 & 9.143864 \\
\hline d223 & 12.1354 & 3.286807 & 3.69 & 0 & 5.69338 & 18.57743 \\
\hline d 224 & 5.38125 & 2.878885 & 1.87 & 0.062 & -0.26126 & 11.02376 \\
\hline d225 & 5.527497 & 2.895627 & 1.91 & 0.056 & -0.14783 & 11.20282 \\
\hline d231 & 3.632378 & 3.391387 & 1.07 & $\odot .284$ & -3.01462 & 10.27937 \\
\hline d232 & 6.245805 & 2.889733 & 2.16 & 0.031 & ๑.582033 & 11.90958 \\
\hline d233 & 4.87267 & 2.872389 & 1.7 & 0.09 & $-\odot .75711$ & 10.50245 \\
\hline d234 & 5.146739 & 2.86956 & 1.79 & 0.073 & $-\odot .4775$ & 10.77097 \\
\hline d235 & 4.783574 & 2.871477 & 1.67 & $\odot .096$ & -0.84442 & 10.41156 \\
\hline $\mathrm{t} 212$ & 0.797173 & 0.954438 & 0.84 & 0.404 & -1.07349 & 2.667836 \\
\hline t213 & $\odot .783776$ & $\odot .955599$ & 0.82 & 0.412 & -1.08916 & 2.656715 \\
\hline $\mathrm{t} 214$ & 0.810881 & $\odot .953742$ & 0.85 & $\odot .395$ & -1.05842 & 2.68018 \\
\hline t215 & 0.774828 & $\odot .957463$ & 0.81 & 0.418 & -1.10177 & 2.651421 \\
\hline t222 & $\odot .629482$ & ๑. 953886 & 0.66 & $\odot .509$ & -1.2401 & 2.499064 \\
\hline t223 & 0.576057 & 0.954651 & 0.6 & 0.546 & -1.29503 & 2.44714 \\
\hline t224 & $\odot .72249$ & ๑. 953831 & 0.76 & 0.449 & -1.14699 & 2.591964 \\
\hline t225 & $\odot .724289$ & $\odot .953799$ & 0.76 & 0.448 & -1.14512 & 2.593701 \\
\hline t231 & -0.43954 & 1.189874 & -0.37 & 0.712 & -2.77165 & 1.89257 \\
\hline t232 & 0.74131 & $\odot .953731$ & 0.78 & 0.437 & -1.12797 & 2.610588 \\
\hline t233 & $\odot .708418$ & $\odot .953723$ & 0.74 & 0.458 & -1.16084 & 2.57768 \\
\hline t234 & $\odot .713307$ & $\odot .953636$ & 0.75 & 0.454 & -1.15578 & 2.582398 \\
\hline t235 & 0.732869 & $\odot .953727$ & 0.77 & 0.442 & -1.1364 & 2.602138 \\
\hline Risk & $\odot .027857$ & ๑. 012704 & 2.19 & $\odot .028$ & ๑. .๑2958 & ๑. 052755 \\
\hline _cons & -4.34676 & 2.900478 & -1.5 & 0.134 & -10.0316 & 1.338073 \\
\hline
\end{tabular}

Marginal effects of risk

\begin{tabular}{rrrrrrr}
\hline & Coef. & Std. Err. & z & P>z & {$[95 \%$ Conf. } & Interval] \\
\hline Risk & 0.027857 & 0.0127 & 2.19 & 0.028 & 0.002958 & 0.052755 \\
\hline
\end{tabular}




\section{Incomplete information}

\begin{tabular}{|c|c|c|c|c|c|c|}
\hline \multicolumn{7}{|c|}{ Log likelihood $=-564.68216$} \\
\hline Choice & Coef. & Std. Err. & $\mathrm{z}$ & $\mathrm{P}>\mathrm{Z}$ & {$[95 \%$ Conf. } & Interval] \\
\hline Period & -0.03954 & 0.028585 & -1.38 & 0.167 & -0.09557 & 0.016485 \\
\hline $\mathrm{d} 1$ & 2.246575 & 1.169403 & 1.92 & $\odot .055$ & -0.04541 & 4.538561 \\
\hline degree2 & 9.844207 & $\odot .898926$ & 10.95 & $\odot$ & 8.082345 & 11.60607 \\
\hline degree3 & 13.70468 & 1.303205 & 10.52 & $\odot$ & 11.15045 & 16.25892 \\
\hline d1_period & -0.0962 & $\odot .04866$ & -1.98 & 0.048 & -0.19157 & -0.00083 \\
\hline d1_degree2 & -5.43655 & 1.117169 & -4.87 & $\odot$ & -7.62616 & -3.24694 \\
\hline d1_degree3 & -4.86144 & 1.554457 & -3.13 & $\odot .002$ & -7.90813 & -1.81476 \\
\hline deg2_period & $-\odot .07148$ & $\odot .0320 \odot 4$ & -2.23 & $\odot .026$ & -0.13421 & $-\odot .00875$ \\
\hline deg3_period & -0.05 & $\odot .043899$ & -1.14 & $\odot .255$ & -0.13604 & $\odot .036 \odot 42$ \\
\hline deg2_per_d1 & $\odot .224834$ & 0.053274 & 4.22 & $\odot$ & 0.120419 & ๑. 329249 \\
\hline deg3_per_d1 & $\odot .222774$ & 0.06418 & 3.47 & $\odot .001$ & $\odot .096984$ & $\odot .348565$ \\
\hline Risk & 0.011876 & 0.012558 & 0.95 & 0.344 & -0.01274 & 0.036489 \\
\hline _cons & -5.29965 & 1.047908 & -5.06 & $\odot$ & -7.35351 & -3.24579 \\
\hline \multicolumn{7}{|c|}{ Marginal effect of risk } \\
\hline Choice & Coef. & Std. Err. & $z$ & $P>Z$ & [95\% Conf. & Interval] \\
\hline risk_0_1 & $4.66 E-05$ & $5.96 E-05$ & 0.78 & 0.435 & $-7 E-05$ & 0.000164 \\
\hline risk_0_2 & $\odot .000597$ & $\odot .00 \odot 709$ & 0.84 & 0.4 & -0.00079 & 0.001986 \\
\hline risk_0_3 & $9.11 E-06$ & $1.29 E-05$ & 0.7 & 0.482 & $-1.6 E-05$ & $3.45 E-05$ \\
\hline risk_1_1 & $6.41 \mathrm{E}-05$ & $\odot .000082$ & 0.78 & 0.434 & $-9.7 E-05$ & $\odot .000225$ \\
\hline risk_1_2 & ๑. 001013 & 0.00118 & 0.86 & $\odot .391$ & -0.0013 & 0.003326 \\
\hline risk_1_3 & $9.90 \mathrm{E}-06$ & 0.000014 & 0.71 & 0.481 & $-1.8 E-05$ & $3.74 \mathrm{E}-05$ \\
\hline
\end{tabular}




\section{Experiment 3}

$\mathrm{n} 2=1$ if network $=2,0$ otherwise

n3 $=1$ if network $=3$, 0 otherwise

$\mathrm{d} 2=1$ if player's degree $=2,0$ otherwise

$\mathrm{d} 3=1$ if player's degree $=3,0$ otherwise

$\mathrm{d} 4=1$ if player's degree $=4,0$ otherwise

$\mathrm{d} 2 \mathrm{n} 2$ : interaction between $\mathrm{d} 2$ and $\mathrm{n} 2$

$\mathrm{d} 2 \mathrm{n} 3$ : interaction between $\mathrm{d} 2$ and $\mathrm{n} 3$

$\mathrm{d} 3 \mathrm{n} 2$ : interaction between $\mathrm{d} 3$ and $\mathrm{n} 2$

$\mathrm{d} 3 \mathrm{n} 3$ : interaction between $\mathrm{d} 3$ and $\mathrm{n} 3$

$\mathrm{d} 4 \mathrm{n} 2$ : interaction between $\mathrm{d} 4$ and $\mathrm{n} 2$

$\mathrm{d} 4 \mathrm{n} 3$ : interaction between $\mathrm{d} 4$ and $\mathrm{n} 3$

$\mathrm{n} 2 \mathrm{p}$ : interaction variable between $\mathrm{n} 2$ and period

$\mathrm{n} 3 \mathrm{p}$ : interaction variable between $\mathrm{n} 3$ and period

$\mathrm{d} 2 \mathrm{p}$ : interaction variable between $\mathrm{d} 2$ and period

$\mathrm{d} 3 \mathrm{p}$ : interaction variable between $\mathrm{d} 3$ and period

$\mathrm{d} 4 \mathrm{p}$ : interaction variable between $\mathrm{d} 4$ and period

d2n2p: interaction variable between $\mathrm{d} 2$, $\mathrm{n} 2$ and period

$\mathrm{d} 2 \mathrm{n} 3 \mathrm{p}$ : interaction variable between $\mathrm{d} 2, \mathrm{n} 3$ and period

$\mathrm{d} 3 \mathrm{n} 2 \mathrm{p}$ : interaction variable between $\mathrm{d} 3, \mathrm{n} 2$ and period

d3n3p: interaction variable between $d 3, n 3$ and period

$\mathrm{d} 4 \mathrm{n} 2 \mathrm{p}$ : interaction variable between $\mathrm{d} 4, \mathrm{n} 2$ and period

$\mathrm{d} 4 \mathrm{n} 3 \mathrm{p}$ : interaction variable between $\mathrm{d} 4, \mathrm{n} 3$ and period

risk_1_1: marginal effect of risk when network 1 and degree==1

risk_1_2: marginal effect of risk when network 1 and degree $==2$

risk_1_3: marginal effect of risk when network 1 and degree $==3$

risk_1_4: marginal effect of risk when network 1 and degree $==4$

risk_2_1: marginal effect of risk when network 2 and degree $==1$

risk_2_2: marginal effect of risk when network 2 and degree $==2$

risk_2_3: marginal effect of risk when network 2 and degree $==3$

risk_2_4: marginal effect of risk when network 2 and degree $==4$

risk_3_1: marginal effect of risk when network 3 and degree==1

risk_3_2: marginal effect of risk when network 3 and degree $==2$

risk_3_3: marginal effect of risk when network 3 and degree $==3$

risk_3_4: marginal effect of risk when network 3 and degree $==4$ 


\begin{tabular}{|c|c|c|c|c|c|c|}
\hline choice & Coef. & Std. Err. & $z$ & $\mathrm{P}>\mathrm{Z}$ & [95\% Conf. & Interval] \\
\hline period & -0.05858 & 0.024295 & -2.41 & 0.016 & -0.10619 & -0.01096 \\
\hline n2 & 1.332241 & 0.784154 & 1.7 & $\odot .089$ & $-\odot .20467$ & 2.869154 \\
\hline n3 & 0.423339 & $\odot .780118$ & 0.54 & 0.587 & -1.10566 & 1.952342 \\
\hline d2 & 4.759135 & 0.528128 & 9.01 & $\odot$ & 3.724024 & 5.794246 \\
\hline d3 & 7.331392 & 0.573277 & 12.79 & $\odot$ & 6.207789 & 8.454995 \\
\hline d4 & 10.60647 & $\odot .856568$ & 12.38 & 0 & 8.927626 & 12.28531 \\
\hline $\mathrm{d} 2 \mathrm{n} 2$ & 0.174066 & 0.832004 & 0.21 & $\odot .834$ & -1.45663 & 1.804763 \\
\hline $\mathrm{d} 2 \mathrm{n} 3$ & 2.272886 & $\odot .879686$ & 2.58 & 0.01 & 0.548733 & 3.997039 \\
\hline$d 3 n 2$ & 21.21926 & 4313.574 & $\odot$ & 0.996 & -8433.23 & 8475.669 \\
\hline d3n3 & 2.881053 & 1.292484 & 2.23 & 0.026 & 0.347831 & 5.414274 \\
\hline $\mathrm{d} 4 \mathrm{n} 2$ & 1.109566 & 2.070312 & 0.54 & 0.592 & -2.94817 & 5.167304 \\
\hline d4n3 & 17.64513 & 4942.264 & $\odot$ & $\odot .997$ & -9669.02 & 9704.305 \\
\hline$n 2 p$ & -0.02345 & $\odot .038179$ & -0.61 & 0.539 & -0.09828 & 0.051381 \\
\hline$n 3 p$ & 0.051894 & 0.031444 & 1.65 & 0.099 & -0.00973 & $\odot .113522$ \\
\hline$d 2 p$ & -0.03878 & $\odot .026727$ & -1.45 & 0.147 & -0.09117 & $\odot .013601$ \\
\hline$d 3 p$ & -0.03067 & 0.027 & -1.14 & 0.256 & -0.08359 & $\odot .022246$ \\
\hline$d 4 p$ & -0.09452 & $\odot .034091$ & -2.77 & $\odot .006$ & $-\odot .16134$ & $-\odot .02771$ \\
\hline$d 2 n 2 p$ & 0.216455 & $\odot .044848$ & 4.83 & $\odot$ & $\odot .128555$ & ๑. 304354 \\
\hline$d 2 n 3 p$ & 0.089878 & $\odot .039223$ & 2.29 & 0.022 & $\odot .0130 \odot 2$ & $\odot .166754$ \\
\hline$d 3 n 2 p$ & 0.101249 & 194.1649 & $\odot$ & 1 & -380.455 & 380.6575 \\
\hline$d 3 n 3 p$ & 0.109483 & 0.068038 & 1.61 & $\odot .108$ & -0.02387 & 0.242834 \\
\hline$d 4 n 2 p$ & 0.277924 & $\odot .140703$ & 1.98 & $\odot .048$ & $\odot . \odot \odot 2151$ & $\odot .553697$ \\
\hline$d 4 n 3 p$ & 0.099375 & 213.2344 & $\odot$ & 1 & -417.832 & 418.0312 \\
\hline risk1 & 0.014794 & $\odot .005353$ & 2.76 & $\odot .0 \odot 6$ & $\odot . \odot \odot 4303$ & 0.025285 \\
\hline _cons & -5.35813 & ๑.596135 & -8.99 & 0 & -6.52653 & -4.18973 \\
\hline
\end{tabular}

Marginal effect of risk

\begin{tabular}{lrrrrrr}
\hline choice & Coef. & Std. Err. & z & P $>$ z & [95\% Conf. & Interval] \\
\hline risk1_1_1 & $4.47 \mathrm{E}-05$ & $2.52 \mathrm{E}-05$ & 1.77 & 0.076 & $-4.72 \mathrm{E}-06$ & $9.42 \mathrm{E}-05$ \\
risk1_1_2 & 0.001786 & 0.000759 & 2.35 & 0.019 & 0.000298 & 0.003274 \\
risk1_1_3 & 0.003011 & 0.001138 & 2.65 & 0.008 & 0.000781 & 0.00524 \\
risk1_1_4 & 0.000718 & 0.000351 & 2.04 & 0.041 & $2.97 \mathrm{E}-05$ & 0.001406 \\
risk1_2_1 & 0.000163 & 0.000133 & 1.23 & 0.22 & $-9.7 \mathrm{E}-05$ & 0.000423 \\
risk1_2_2 & 0.003687 & 0.001344 & 2.74 & 0.006 & 0.001052 & 0.006322 \\
risk1_2_3 & $8.74 \mathrm{E}-13$ &. &. &. & &. \\
risk1_2_4 & $5.34 \mathrm{E}-05$ & $9.63 \mathrm{E}-05$ & 0.55 & 0.58 & -0.00014 & 0.000242 \\
risk1_3_1 & $7.17 \mathrm{E}-05$ & $5.58 \mathrm{E}-05$ & 1.29 & 0.199 & $-3.8 \mathrm{E}-05$ & 0.000181 \\
risk1_3_2 & 0.002873 & 0.001327 & 2.17 & 0.03 & 0.000273 & 0.005473 \\
risk1_3_3 & 0.000179 & 0.000196 & 0.91 & 0.361 & -0.00021 & 0.000563 \\
risk1_3_4 & $9.74 \mathrm{E}-12$ &. &. &. & &. \\
\hline
\end{tabular}




\section{Online Supplement C: Experimental instructions}

\section{I) Complete Information - Substitutes (Experiment 1)}

[Note: The corresponding instructions for Experiment 2 are analogous (it just changes the three networks)]

The aim of this experiment is to study how individuals make decisions in certain contexts. The instructions are simple. You first receive the instructions for Part 1 of the experiment, after which you will receive instructions for a second part that is independent of Part 1.If you follow the instructions carefully you will earn a non-negligible amount of money in cash (Euros) at the end of the experiment. During the experiment, your earnings will be accounted in ECU (Experimental Currency Units). Individual payments will remain private, as nobody will know the other participants' payments. Any communication among you is strictly forbidden and will result in an immediate exclusion from the experiment.

1.- The experiment consists of 40 periods. Please note that there will be 5 unpaid trial periods before the 40 periods start. In each period you will be randomly assigned to a group of 5 participants. This group is determined randomly at the beginning of the period. Therefore, the group you are assigned to changes at each period. In this room, there are 10 participants (including yourself) that are potential members of your group. That is, at every period your group of 5 participants is selected among these 10 participants, each of them being equally likely to be in your group. You will not know the identities of any of these participants.

2.- At each period, the computer selects randomly a network for your group: the orange network, the green network or the purple network:
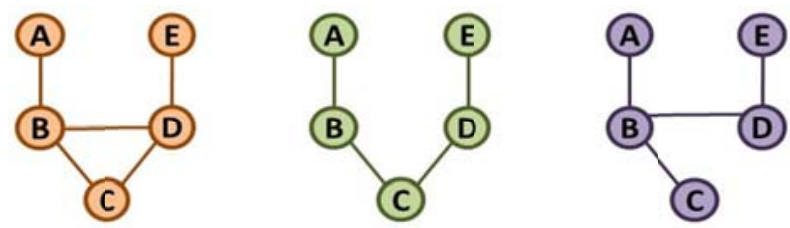

\section{Orange network Green network Purple network}

Once a network is selected, you (and the other members of your group) are randomly assigned to a position: A, B, C, D or E, all of them being equally likely. The assignment process is random: In each period, you are equally likely to be located in each of the 5 positions. At each period, you will be informed of the selected network (color) and of your position (letter).

In a network, a link is represented by a line (connection) between two positions. For example, in the orange network, position $\mathbf{B}$ has three links: it is linked to positions $\mathbf{A}, \mathbf{C}$ and $\mathbf{D}$ (but it is not linked to position E). Summarizing:

- In the orange network there are two positions with 1 link (positions $\mathbf{A}$ and $\mathbf{E}$ ), one position

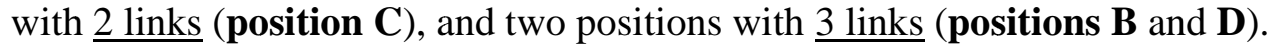

- In the green network there are two positions with 1 link (positions $\mathbf{A}$ and $\mathbf{E}$ ), three positions with 2 links (positions B, $\mathbf{C}$ and $\mathbf{D}$ ), and no position with $\underline{3 \text { links. }}$ 
- In the purple network there are three positions with 1 link (positions $\mathbf{A}, \mathbf{C}$ and $\mathbf{E}$ ), one position with 2 links (position D), and one position with $\underline{3 \text { links (position B). }}$

You can notice that both the green and the purple network have one link less that the orange one: In the green network positions $\mathbf{B}$ and $\mathbf{D}$ are not linked, and in the purple network positions $\mathbf{C}$ and $\mathbf{D}$ are not linked.

Your earnings of the period can only be affected by your decisions and the decisions of those participants located in positions that are linked to yours, as specified below.

3.- In each period, knowing the selected network and your position, you will be asked to make a choice: to be ACTIVE or INACTIVE (the other participants are asked to make the same choice). Your payoff of the period will depend on your choice and on the choices of those participants of your group located in positions linked to yours: You earn 100 ECU if either you or at least one of the participants located in positions linked to yours choose to be ACTIVE. Being active has a cost of 50 ECU. Hence,

- If you choose to be ACTIVE your period payoff is $\mathbf{5 0}$ ECU for sure [100 - 50]

- If you choose to be INACTIVE your period payoff can be:

$>100$ ECU if at least one participant linked to you chooses to be ACTIVE, or

0 ECU if no participant linked to you chooses to be ACTIVE.

4.- At the end of every period, you will get information about current and past periods. The information consists of:

- The selected network.

- Your position in the network.

- Your choice (ACTIVE or INACTIVE).

- The number of participants linked to you that chose to be ACTIVE.

- Your (period) payoff.

5.- Payoffs. At the end of the experiment, you will be paid the earnings that you achieved in 4 periods, that will be randomly selected across the 40 periods of play (all periods selected will have the same probability). These earnings are transformed to cash at the exchange rate of $\mathbf{2 0}$ $\mathbf{E C U}=\mathbf{1} €$. In addition, just by showing up, you will also be paid a fee of $\mathbf{5} €$. 


\section{II) Incomplete Information - Complements $\mathbf{- ~} \mathbf{p}=\mathbf{0 . 8}$ (Experiment 1)}

[Note: The case $p=0.2$ is analogous (it just changes the virtual urn composition). The corresponding instructions for Experiment 2 are also analogous (it just changes the three networks)]

The aim of this experiment is to study how individuals make decisions in certain contexts. The instructions are simple. You first receive the instructions for Part 1 of the experiment, after which there you will receive instructions for a second part that is independent of Part 1. If you follow the instructions carefully you will earn a non-negligible amount of money in cash (Euros) at the end of the experiment. During the experiment, your earnings will be accounted in ECU (Experimental Currency Units). Individual payments will remain private, as nobody will know the other participants' payments. Any communication among you is strictly forbidden and will result in an immediate exclusion from the experiment.

1.- The experiment consists of 40 periods. Please note that there will be 5 unpaid trial periods before the 40 periods start. In each period you will be randomly assigned to a group of 5 participants. This group is determined randomly at the beginning of the period. Therefore, the group you are assigned to changes at each period. In this room, there are 10 participants (including yourself) that are potential members of your group. That is, at every period your group of 5 participants is selected among these 10 participants, each of them being equally likely to be in your group. You will not know the identities of any of these participants.

2.- At each period, the computer selects one color from a virtual urn. The virtual urn contains 10 balls: 8 orange balls, 1 green ball and 1 purple ball.

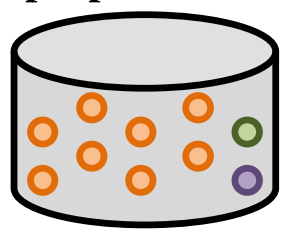

All the 10 balls of the virtual urn are equally likely to be selected by the computer. The color of the selected ball determines a network for your group: the orange network, the green network or the purple network. Once the network has been selected, the ball is returned to the virtual urn. Thus, in each period the color selection process is identical (there are always 8 orange balls, 1 green ball and 1 purple ball, and one of them is randomly picked by the computer). The three possible networks are:
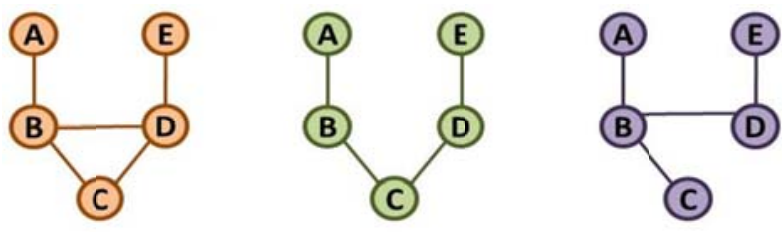

\section{Orange network Green network Purple network}

Once a network is selected, you (and the other members of your group) are randomly assigned to a position: A, B, C, D or E, all of them being equally likely. The assignment process is random: 
At each period, you are equally likely to be located in each of the 5 positions. At each period, you will neither be informed of the selected network (color) nor of your position (letter).

In a network, a link is represented by a line (connection) between two positions. For example, in the orange network, position $\mathbf{B}$ has three links: it is linked to positions $\mathbf{A}, \mathbf{C}$ and $\mathbf{D}$ (but it is not linked to position E). Summarizing:

- In the orange network there are two positions with 1 link (positions $\mathbf{A}$ and $\mathbf{E}$ ), one position

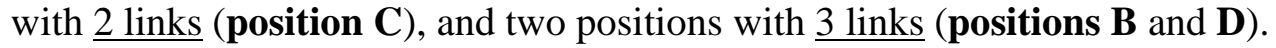

- In the green network there are two positions with 1 link (positions $\mathbf{A}$ and $\mathbf{E}$ ), three position with 2 links (positions B, $\mathbf{C}$ and $\mathbf{D}$ ), and no position with $\underline{3 \text { links. }}$

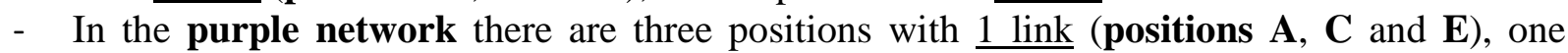

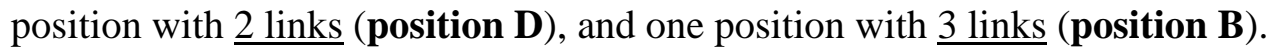

You can notice that both the green and the purple network have one link less that the orange one: In the green network positions $\mathbf{B}$ and $\mathbf{D}$ are not linked, and in the purple network positions $\mathbf{C}$ and $\mathbf{D}$ are not linked.

Your earnings of the period can only be affected by your decisions and the decisions of those participants located in positions that are linked to yours, as specified below.

3.- In each period, you will only be informed about how many links your assigned position has (1 link, 2 links or $\underline{3 \text { links) }}$ in the selected network, but you will neither know with certainty which is the selected network nor your exact position.

For example, if at a particular period you are informed that your position has $\underline{3 \text { links, }}$, there are different paths that could lead to this outcome: It may be the case that the selected network is the orange network and you have been assigned to position $\mathbf{B}$ or $\mathbf{D}$, or it may be the case that the selected network is the purple network and you have been assigned to position $\mathbf{B}$.

4.- In each period, you will be asked to make a choice: to be ACTIVE or INACTIVE (the other participants are asked to make the same choice). Your payoff of the period will depend on your choice and on the choices of those participants of your group located in positions linked to yours. If you choose to be INACTIVE, your period payoff is 50 ECU. If you choose to be ACTIVE, your period payoff is calculated as follows: First, add 100 ECU per participant linked to you that also chooses to be ACTIVE; then, divide the result by 3. Hence,

- If you choose to be ACTIVE your period payoff can be:

$>$ 100.00 ECU if 3 participants linked to you choose to be ACTIVE $[\underline{100+100+1003}]$, or

$>66.66 \mathrm{ECU}$ if 2 participants linked to you choose to be ACTIVE $\left[\frac{100+100}{3}\right]$, or

$>$ 33.33 ECU if 1 participants linked to you chooses to be ACTIVE $\left[\frac{100}{3}\right]$, or

0.00 ECU if no participant linked to you chooses to be ACTIVE.

- If you choose to be INACTIVE your period payoff is 50.00 ECU for sure.

5.- At the end of every period, you will get information about current and past periods. The information consists of: 
- The selected network.

- Your position in the network.

- Your choice (ACTIVE or INACTIVE).

- The number of participants linked to you that chose to be ACTIVE.

- Your (period) payoff.

6.- Payoffs. At the end of the experiment, you will be paid the earnings that you achieved in 4 periods, that will be randomly selected across the 40 periods of play (all periods will be selected with the same probability). These earnings are transformed to cash at the exchange rate of $\mathbf{2 0}$ $\mathbf{E C U}=\mathbf{1} €$. In addition, just by showing up, you will also be paid a fee of $\mathbf{5} €$. 


\section{III) Network 1 (Experiment 3)}

[Note: The corresponding instructions for Network 2 and Network 3 are analogous (they just have a different depiction of the network)]

The aim of this experiment is to study how individuals make decisions in certain contexts. The instructions are simple. First you get the instructions for Part 1 of the experiment, after which there will be a second part that is independent of Part 1. If you follow the instructions carefully you will earn a non-negligible amount of money in cash (Euros) at the end of the experiment. During the experiment, your earnings will be accounted in ECU (Experimental Currency Units). Individual payments will remain private, as nobody will know the other participants' payments. Any communication among you is strictly forbidden and will result in an immediate exclusion from the experiment.

1.- The experiment consists of $\mathbf{4 0}$ periods, and there are $\mathbf{2 0}$ participants, including yourself. The participants will remain the same throughout the experiment. Please note that there will be 5 unpaid trial periods before the 40 periods start. At each period, you and each of the remaining nineteen participants will be assigned one position of the following NETWORK. The positions in the network are numbered from 1 to 20.

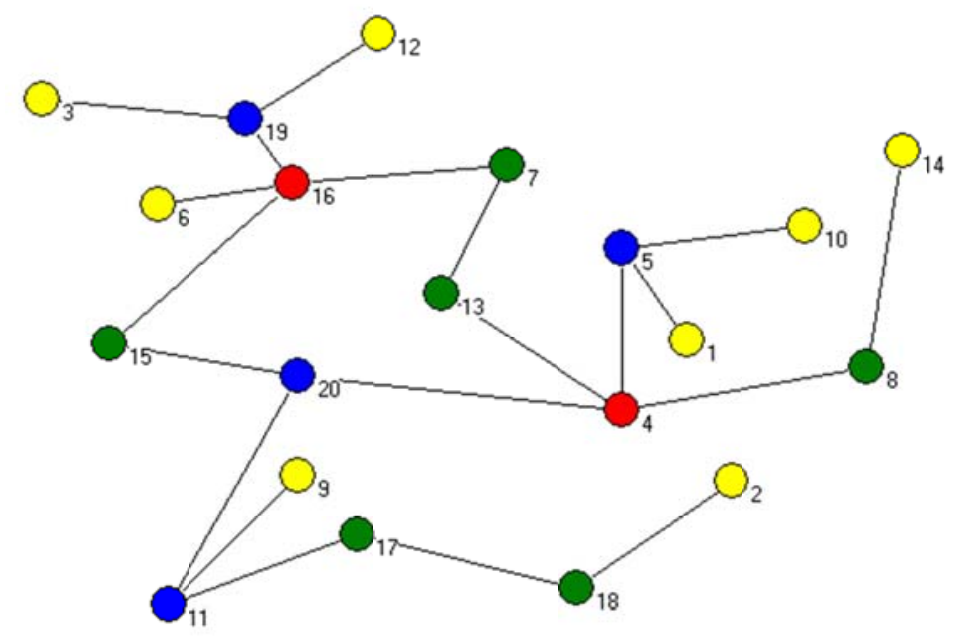

2.- In the network, a link is represented by a line (connection) between two positions. For example, position 16 has four links: it is linked to positions 6, 7, 15 and 19 (but it is not linked to the remaining positions).

Note that there are four classes of positions in the network, identified by different colors.

- There are eight yellow positions: Those positions with one link (1, 2, 3, 6, 9, 10, 12 and 14).

- There are six green positions: Those positions with two links (7, 8, 13, 15, 17 and 18). 
- There are four blue positions: Those positions with three links (5, 11, 19 and 20).

- There are two red positions: Those positions with four links (4 and 16).

3.- In each period, you (and the other participants) are randomly assigned by the computer to a position from 1 to 20 in the network, all of them being equally likely. The assignment process is random: At each period, you are equally likely to be located in each of the 20 positions of the network.

3.- In each period, you will only be informed of the color of your position, that is, you will know how many links your assigned position has: 1 link (yellow), 2 links (green), 3 links (blue) or 4 links (red). However, you will not be informed of which is your exact position.

For example, if in a particular period you are informed that your position has 3 links (blue), then you know that you can be in position $5,11,19$ or 20 , and that you can be in any of them with the same probability. Note that, in such a case, you also know that you cannot be in a yellow, green or red position.

Your earnings of the period can only be affected by your decisions and the decisions of those participants located in positions that are linked to yours, as specified below.

4.- In each period, knowing the selected network and your position, you will be asked to make a choice: to be ACTIVE or INACTIVE (the other participants are asked to make the same choice). Your payoff of the period will depend on your choice and on the choices of those participants located in positions linked to yours. If you choose to be INACTIVE, your period payoff is 50 ECU. If you choose to be ACTIVE, your period payoff is calculated as follows: First, add 100 ECU per participant linked to you that also chooses to be ACTIVE; then, divide the result by 3 . Hence,

- If you choose to be ACTIVE your period payoff can be:

$>$ 133.33 ECU if 4 participants linked to you choose to be ACTIVE $\left[\frac{100+100+100+100}{3}\right]$, or

100.00 ECU if 3 participants linked to you choose to be ACTIVE $\left[\frac{100+100+100}{3}\right]$, or

66. $66 \mathrm{ECU}$ if 2 participants linked to you choose to be ACTIVE $\left[\frac{100+100}{3}\right]$, or

33. 33 ECU if 1 participants linked to you chooses to be ACTIVE $\left[\frac{100}{3}\right]$, or

0. 00 ECU if no participant linked to you chooses to be ACTIVE. 
- If you choose to be INACTIVE your period payoff is 50.00 ECU for sure.

5.- At the end of every period, you will get information about current and past periods. The information consists of:

- Your position in the network.

- Your choice (ACTIVE or INACTIVE).

- The number of participants linked to you that chose to be ACTIVE.

- Your (period) payoff.

6.- Payoffs. At the end of the experiment, you will be paid the earnings that you achieved in 4 periods, that will be randomly selected across the 40 periods of play (all periods will be selected with the same probability). These earnings are transformed to cash at the exchange rate of 20 ECU $=1 €$. In addition, just by showing up, you will also be paid a fee of $5 €$. 


\section{Part 2 of the experiment}

[Note: These are the instructions for the elicitation of risk attitudes. They were used at the end of all experimental sessions]

In part 2 you need to make 12 decisions. In each decision, your choice can only have monetary consequences for you, but not for any other participant.

1) The first decision is as follows:

You have $100 \mathrm{ECU}$, of which you can invest as many as you like into a risky option. Your investment will be successful with 50\% chance. If successful, you get 2.5 times the invested amount back. If not successful, you lose your invested amount of ECU. All ECU that you do not invest are for you to keep.

How many ECU would you like to invest into the risky option?

2) The other 11 decisions are fairly similar.

In each decision you can choose between an option LEFT and an option RIGHT. Your payoff from choosing option LEFT depends on in each of the 11 decisions on chance. Choosing option RIGHT, however, implies a safe payoff. For instance, you could be asked whether you prefer an option LEFT with a 50\% chance of getting 100 ECU and a 50\% chance of getting 0 ECU or an option RIGHT in which you get $c$ ECU for sure. The value $c$ can take on different, positive values that you will see on the experimental screen once this part starts. In each of the 11 decisions you will then need to choose one of the two options. The decision problem will be presented on the screen as follows:

\begin{tabular}{|c|c|c|}
\hline LEFT & RIGHT & Your choice \\
\hline $\begin{array}{c}\text { with } 50 \% \text { chance } 100 \mathrm{ECU} \\
\text { and }\end{array}$ & c ECU for sure & LEFT $\bigcirc \bigcirc$ RIGHT \\
with $50 \%$ chance $0 \mathrm{ECU}$ & & \\
\hline
\end{tabular}

3) Payoff from this part: At the end of the experiment one of your 12 decisions will be randomly selected for payment.

If, for instance, your first decision is selected, then you get with $50 \%$ chance 2.5 times the invested amount, in addition to the amount of ECU that you kept.

If any of the other 11 decisions is selected, is the option that you chose payoff-relevant. If you have chosen the risky option, then you get with 50\% chance 100 ECU and with $50 \%$ chance 0 ECU. If you have chosen the safe amount, then you get the amount that was stated in that decision.

As in Part 1 the exchange rate is: 20 ECU $=1$ Euro.

After this part, the experiment ends. 


\section{Online Supplement D: Generalized Risk-Dominance}

To study (ordinal) Generalized Risk-dominance, we use the concepts (and notation) introduced by Peski (2010). We focus on the scenarios with multiplicity of equilibria. Let $N$ be the set of players and let $a=\left(a_{1}, \ldots, a_{n}\right)$ be an action (strategy) profile.

Definition 1. Given an action profile $a$, two action profiles, $\eta$ and $\bar{\eta}$, are $a$-associated if, for each $i \in N$, either $\eta_{i}=a_{i}$ or $\bar{\eta}_{i}=a_{i}$

Definition 2. An action profile $a$ is ordinal GR-dominant if, for each player $i$, and for each pair of $a$-associated action profiles, $\eta$ and $\bar{\eta}, a_{i}$ is a best response of player $i$ to either $\eta$ or $\bar{\eta}$.

\section{I) Complete information scenario}

We denote by $N_{i}$ the set of player that have a link to player $i$ and $n_{i}=\left|N_{i}\right|$. For each $i \in N$, $a_{i} \in\{0,1\}$, where 0 means inactive and 1 means active.

\section{I.i) Strategic substitutes}

Lemma 1. Consider the game of strategic substitutes defined in Section 3 under the complete information scenario. If an action profile $a$ is such that there exists $i \in N$ such that (i) $n_{i}>1$, and (ii) $a_{i}=1$, then $a$ is not ordinal GR-dominant.

Proof: Let $j, j^{\prime} \in N_{i}$. There exist two $a$-associated action profiles, $\eta$ and $\bar{\eta}$, such that $\eta_{j}=$ $1, \eta_{j \prime}=0, \bar{\eta}_{j}=0, \bar{\eta}_{j^{\prime}}=1$. It clearly follows that $a_{i}=1$ is neither a best response of player $i$ to $\eta$ nor a best response to $\bar{\eta}$. QED

Lemma 2. Consider the game of strategic substitutes defined in Section 3 under the complete information scenario. If each player with degree higher than 1 is linked to at least one player with degree 1 and each player with degree 1 is linked to one player with degree higher than 1 , then an action profile a such that, for each $i \in N, a_{i}=1$ if and only if $n_{i}=1$ is ordinal GRdominant.

Proof: Consider action profile $a$, and $i \in N$ such that $n_{i}=1$, and let $j \in N_{i}$. Then $a_{i}=1$ and $a_{j}=0$. Thus, for each pair of $a$-associated action profiles, $\eta$ and $\bar{\eta}$, it cannot be the case that $\eta_{j}=\bar{\eta}_{j}=1$. It follows that $a_{i}=1$ is a best response of player $i$ to at least one of the two profiles $(\eta, \bar{\eta})$. Now consider $j \in N$ such that $n_{j}>1$ and let $i \in N_{j}$ such that $n_{i}=1$. Then $a_{j}=0$ and $a_{i}=1$. Thus, for each pair of $a$-associated action profiles, $\eta$ and $\bar{\eta}$, it cannot be the 
case that $\eta_{i}=\bar{\eta}_{i}=0$. It follows that $a_{j}=0$ is a best response of player $i$ to at least one of the two profiles $(\eta, \bar{\eta})$. QED

Proposition 7. Consider the game of strategic substitutes defined in Section 3 with complete information, and consider any of the three networks of Experiment 1 . In the Purple network there only exists an ordinal GR-dominant equilibrium, and it is the most secure equilibrium $A C E / B D$. In the other two networks (Orange and Green) no equilibrium is ordinal GR-dominant.

Proof: First consider the Orange network. In the equilibria ACE/BD, BE/ACD and AD/BCE, respectively, the player in position $\mathrm{C}, \mathrm{B}$ and $\mathrm{D}$ satisfies the conditions (i)-(ii) of Lemma 1. Hence, none of the equilibria is ordinal GR-dominant. Now consider the Green network. In the equilibria $\mathrm{ACE} / \mathrm{BD}, \mathrm{BD} / \mathrm{ACE}, \mathrm{BE} / \mathrm{ACD}$ and $\mathrm{AD} / \mathrm{BCE}$, respectively, the player in position $\mathrm{C}, \mathrm{B}$, $\mathrm{B}$ and $\mathrm{D}$ satisfies the conditions (i)-(ii) of Lemma 1. Hence, none of the equilibria is ordinal GRdominant. Finally, consider the Purple network. In the equilibria ACD/BE and BE/ACD, respectively, the player in position $\mathrm{D}$ and $\mathrm{B}$ satisfies the conditions (i)-(ii) of Lemma 1. Therefore, none of them is ordinal GR-dominant. In contrast, the equilibrium ACE/BD is ordinal GR-dominant, since it satisfies the conditions of Lemma 2. QED

\section{I.ii) Strategic Complements}

Lemma 3. Consider the game of strategic complements defined in Section 3 under the complete information scenario. If an action profile $a$ is such that there exists $i \in N$ such that (i) $n_{i}>1$, (ii) $a_{i}=1$ and (iii) $\left|\left\{j \in N_{i}: a_{j}=1\right\}\right|<3$, then a is not ordinal GR-dominant.

Proof. Consider action profile $a$. First, let $i \in N$ be such that $\left|\left\{j \in N_{i}: a_{j}=1\right\}\right|<2$. Then $a_{i}=1$ provides a payoff of at most $100 / 3<50$. Thus, it is not a best response to any action profile. Now, consider the case $\left|\left\{j \in N_{i}: a_{j}=1\right\}\right|=2$, and let $j, j^{\prime} \in N_{i}$ be such that $a_{j}=a_{j \prime}=1$. There exist two $a$-associated action profiles, $\eta$ and $\bar{\eta}$, such that $\eta_{j}=1, \eta_{j^{\prime}}=0, \bar{\eta}_{j}=0, \bar{\eta}_{j^{\prime}}=1$ and that, for any $j^{\prime \prime} \in N_{i} \backslash\left\{j, j^{\prime}\right\}, \eta_{j \prime \prime}=\bar{\eta}_{j^{\prime \prime}}=0$. It clearly follows that $a_{i}=1$ is neither a best response of player $i$ to $\eta$ nor a best response to $\bar{\eta}$, since in both cases it provides a payoff of $100 / 3<50$. QED

Lemma 4. Consider the game of strategic complements defined in Section 3 under the complete information scenario. If the network is such that, for each $i \in N, n_{i}<4$, then the action profile a with $a_{i}=0$ for all $i \in N$ is ordinal GR-dominant.

Proof. Consider, for the sake of contradiction, that given two $a$-associated action profiles, $\eta$ and $\bar{\eta}, a_{i}=0$ is not a best response to any of them. The fact that $a_{i}=0$ is not a best response to $\eta$ implies that $n_{i} \geq 2$ and $\left|\left\{j \in N_{i}: \eta_{j}=1\right\}\right| \geq 2$. But, since $\eta$ and $\bar{\eta}$ are $a$-associated and $n_{i}<4$, it 
follows that $\left|\left\{j \in N_{i}: \bar{\eta}_{j}=1\right\}\right| \leq 1$, a contradiction with the fact that $a_{i}=0$ is not a best response to $\bar{\eta}$. QED

Proposition 8. Consider the game of strategic complements defined in Section 3 with complete information. In any of the networks of Experiments 1 and 2 with multiple equilibria, the secure equilibrium is GR-dominant and the efficient equilibrium is not.

Proof: We first note that, in all the scenarios, the secure equilibrium (all players inactive) satisfies the conditions of Lemma 4 and, therefore, it is ordinal GR-dominant. We now prove that the efficient equilibrium of each scenario is not ordinal GR-dominant by identifying a player position that satisfies the conditions of Lemma 3. In the BCD/AE equilibrium of the Orange network such a player position is $\mathrm{B}$. In the BCDE/A equilibrium of the Blue network such a player position is $\mathrm{B}$. In the $\mathrm{CDE} / \mathrm{AB}$ equilibrium of the Blue network such a player position is $\mathrm{C}$. In the BCDE/A equilibrium of the Brown network such a player position is B. QED

\section{II) Incomplete information scenario}

\section{II.i) Strategic complements -5 player networks}

Since in our set of 5-player networks, the maximum degree is 3, for each player $i \in N, a_{i}=$ $\left(a_{i, 1}, a_{i, 2}, a_{i, 3}\right) \in\{0,1\}^{3}$. For each $k \in\{1,2,3\}, a_{i, k}=0 \quad\left(a_{i, k}=1\right)$ represents the choice of inactive (active) in the event in which player $i$ has degree $k$.

Proposition 9. Consider the game of strategic complements defined in Section 3 under any of the network generating processes of either Experiment 1 or Experiment 2 with incomplete information. The secure equilibrium is ordinal GR-dominant and the efficient equilibrium is not.

Proof. Part 1. We first show that the action profile $a$ such that for each $i \in N, a_{i}=(0,1,1)$ (i.e., the efficient equilibrium) is not ordinal GR-dominant. Consider, without loss of generality, player 1 . There exist two $a$-associated action profiles $\eta$ and $\bar{\eta}$ such that $\eta_{2}=\eta_{3}=(0,1,1)$ and $\eta_{4}=\eta_{5}=(0,0,0)$, and $\bar{\eta}_{2}=\bar{\eta}_{3}=(0,0,0)$ and $\bar{\eta}_{4}=\bar{\eta}_{5}=(0,1,1)$. Since players are randomly allocated in the (selected) network with uniform probability, $a_{1}$ is a best response to $\eta$ if and only if it is a best reponse to $\bar{\eta}$. Consider the choice of player 1 in the event in which $n_{1}=2$, $a_{1,2}=1$. Let a profile $\eta^{\prime}$ be such that $\eta_{2}^{\prime}=\eta_{3}^{\prime}=(1,1,1)$ and $\eta_{4}^{\prime}=\eta_{5}^{\prime}=(0,0,0)$, i.e., players 2 and 3 ( 4 and 5 ) are always active (inactive) regardless of their degree. Since the incentives to be active are (weakly) increasing in the activity levels of other players, in order to show that $a_{1,2}=1$ is not a best response to $\eta$, it suffices to show that $a_{1,2}=1$ is not a best response to $\eta^{\prime}$. Thus, under profile $\eta^{\prime}$, the probability that player 1 has $k \in\{0,1,2\}$ active neighbors is $p_{k}=$ 
$\frac{\left(\begin{array}{l}2 \\ k\end{array}\right) \cdot\left(\begin{array}{c}2 \\ 2-k\end{array}\right)}{\left(\begin{array}{l}4 \\ 2\end{array}\right)}$. Thus, in such a case, the expected payoff to player 1 is $\sum_{k=0}^{k=2} p_{k} \cdot k \cdot \frac{100}{3}=\frac{100}{3}<50$. It follows that $a_{1,2}=1$ is not a best response to $\eta^{\prime}$. Thus it is nor neither a best response to $\eta$ nor to $\bar{\eta}$ and, it follows that $a$ is not ordinal GR-dominant.

Part 2. We now show that the action profile $a$ such that for each $i \in N, a_{i}=(0,0,0)$ (i.e., the secure equilibrium) is ordinal GR-dominant. Consider, without loss of generality, player 1 . There exist two $a$-associated action profiles $\eta$ and $\bar{\eta}$ such that $\eta_{2}=\eta_{3}=(0,0,0)$ and $\eta_{4}=\eta_{5}=$ $(1,1,1)$, and $\bar{\eta}_{2}=\bar{\eta}_{3}=(1,1,1)$ and $\bar{\eta}_{4}=\bar{\eta}_{5}=(0,0,0)$. In each profile there are two players in $N \backslash\{1\}$ that are always active (regardless of their degree), and two players in $N \backslash\{1\}$ that are always inactive. Since players are randomly allocated in the selected network with uniform probability, $a_{1}$ is a best response to $\bar{\eta}$ if and only if it is a best response to $\eta$.

We now prove that $a_{1}=(0,0,0)$ is a best response to $\eta$. We check it entry by entry. First, consider the event in which $n_{1}=1$. Clearly, $a_{1,1}=0$ is a best response. Second, consider the event in which $n_{1}=2$. Under profile $\eta$, the probability that player 1 has $k \in\{0,1,2\}$ active neighbors is $p_{k}=\frac{\left(\begin{array}{l}2 \\ k\end{array}\right) \cdot\left(\begin{array}{c}2 \\ 2-k\end{array}\right)}{\left(\begin{array}{l}4 \\ 2\end{array}\right)}$. Thus, in such a case, the payoff to player 1 by choosing $a_{1,2}=0$ (i.e., 50) exceeds $\sum_{k=0}^{k=2} p_{k} \cdot k \cdot \frac{100}{3}=\frac{100}{3}$, which is the expected payoff he would get by choosing action 1 . Finally, consider the event in which $n_{1}=3$. Under profile $\eta$, with probability $1 / 2$ player 1 has two active neighbors and one inactive neighbor, and with probability $1 / 2$ player 1 has one active neighbor and two inactive neighbors. Thus, in such a case, the payoff to player 1 by choosing $a_{1,2}=0$ (i.e., 50) equals the expected payoff he would get by choosing action 1 $\left(\frac{1}{2} \cdot \frac{100}{3}+\frac{1}{2} \cdot \frac{200}{3}\right)$ and, therefore, it is also a best response.

We now claim that, for any possible pair $\left(\eta^{\prime}, \bar{\eta}^{\prime}\right)$ of $a$-associated action profiles, then $a_{1}=(0,0,0)$ is a best response to at least one of the action profiles $\eta^{\prime}$ and $\bar{\eta}^{\prime}$. To see this, note that by the definition of $a$-associated action profiles, at least one of the profiles $\eta^{\prime}$ and $\bar{\eta}^{\prime}$ must have two or more players choosing $(0,0,0)$. In such a profile, say $\eta^{\prime}$, the activity level in any action chosen by each of the remaining players -those not choosing $(0,0,0)$ - is necessarily (weakly) lower than that of the full activity action $(1,1,1)$. Since in profile $\eta$ exactly two players choose action $(0,0,0)$ and the remaining players choose $(1,1,1)$, and the incentives to play $(0,0,0)$ are (weakly) decreasing in the activity levels of the other players, the claim follows. This completes the proof. QED

\section{II.ii) Strategic complements - 20-player networks}

Since in our set of 20-player networks, the maximum degree is 4, for each player $i \in N, a_{i}=$ $\left(a_{i, 1}, a_{i, 2}, a_{i, 3}, a_{i, 4}\right) \in\{0,1\}^{4}$. For each $k \in\{1,2,3,4\}, a_{i, k}=0\left(a_{i, k}=1\right)$ represents the choice of inactive (active) in the event in which player $i$ has degree $k$. 
Proposition 10. Consider the game of strategic complements defined in Section 3 under any of the network generating processes of Experiment 3. None of the equilibrium profiles is ordinal GR-dominant.

Proof. Part 1. We first show that the action profile $a$ such that for each $i \in N, a_{i}=(0,1,1,1)$ is not ordinal GR-dominant. Consider, without loss of generality, player 1 . There exist two aassociated profiles, $\eta$ and $\bar{\eta}$, such that, for each $i \in\{2, \ldots, 10\}, \eta_{i}=(0,0,0,0)$ and $\bar{\eta}_{i}=(0,1,1,1)$ and, for each $j \in\{11, \ldots, 20\}, \eta_{j}=(0,1,1,1)$ and $\bar{\eta}_{j}=(0,0,0,0)$. Consider the event in which $n_{1}=2$. Clearly, if $a_{1,2}=1$ is not a best response to profile $\eta$, it will neither be to profile $\bar{\eta}$, since the later one has one more player choosing the full-inactivity strategy (10 vs. 9 players $)$ and all the remaining players choosing the same strategy $(0,1,1,1)$ (recall that all players are randomly allocated in the network with uniform probability). Thus, to prove the result it suffices to show that $a_{1,2}=1$ is not a best response to profile $\eta$.

To this aim, consider a profile $\eta^{\prime}$ such that, for each $i \in\{2, \ldots, 10\}, \eta_{i}^{\prime}=(0,0,0,0)$ and, for each $j \in\{11, \ldots, 20\}, \eta_{i}^{\prime}=(1,1,1,1)$. In profile $\eta^{\prime}$ there are ten players in $N \backslash\{1\}$ that are always active (regardless of their degree), and nine players in $N \backslash\{1\}$ that are always inactive. It is straightforward to see that if $a_{1,2}=1$ is not a best response to profile $\eta^{\prime}$, it cannot be a best response to profile $\eta$. Hence, it suffices to prove that $a_{1,2}=1$ is not a best response to profile $\eta^{\prime}$. Under profile $\eta^{\prime}$, when $n_{1}=2$, the probability that player 1 has $k \in\{0,1,2\}$ active neighbors is $p_{k}^{\prime}=\frac{\left(\begin{array}{c}10 \\ k\end{array}\right) \cdot\left(\begin{array}{c}9 \\ 2-k\end{array}\right)}{\left(\begin{array}{c}19 \\ 2\end{array}\right)}$. Thus, in such a case, the expected payoff to player 1 by choosing $a_{1,2}=1$ is $\sum_{k=0}^{k=2} p_{k}^{\prime} \cdot k \cdot \frac{100}{3}=35.1<50$. Thus $a_{1,2}=1$ is not a best response to profile $\eta^{\prime}$. It follows that $a_{1,2}$ (and, therefore, $a_{1}$ ) is neither a best response to $\eta$ nor to $\bar{\eta}$ and, thus, $a$ is not ordinal GRdominant.

Part 2. We now show that the action profile $a$ such that for each $i \in N, a_{i}=(0,0,0,0)$ is not ordinal GR-dominant. Consider, without loss of generality, player 1 . There exist two aassociated profiles, $\eta$ and $\bar{\eta}$, such that, for each $i \in\{2, \ldots, 10\}, \eta_{i}=(1,1,1,1)$ and $\bar{\eta}_{i}=(0,0,0,0)$ and, for each $j \in\{11, \ldots, 20\}, \eta_{j}=(0,0,0,0)$ and $\bar{\eta}_{j}=(1,1,1,1)$. In profile $\eta$ (profile $\bar{\eta}$ ) there are nine (ten) players in $N \backslash\{1\}$ that are always active (regardless of their degree), and ten (nine) players in $N \backslash\{1\}$ that are always inactive. Consider the event in which $n_{1}=4$. Clearly, if $a_{1,4}=0$ is not a best response to profile $\eta$, it will neither be to profile $\bar{\eta}$ (recall that all players are randomly allocated in the network with uniform probability). Thus, to prove the result it suffices to show that $a_{1,4}=0$ is not a best response to profile $\eta$.

Under profile $\eta$, when $n_{1}=4$, the probability that player 1 has $k \in\{0,1,2,3,4\}$ active neighbors is $q_{k}=\frac{\left(\begin{array}{c}9 \\ k\end{array}\right) \cdot\left(\begin{array}{c}10 \\ 4-k\end{array}\right)}{\left(\begin{array}{c}19 \\ 4\end{array}\right)}$. Thus, in such a case, the payoff to player 1 by choosing $a_{1,4}=0$ (i.e., 50) is lower than the expected payoff he would get by choosing action 1 , i.e., $\sum_{k=0}^{k=4} q_{k} \cdot k \cdot \frac{100}{3}=63.2$. 
It follows that $a_{1,4}$ (and, therefore, $a_{1}$ ) is neither a best response to $\eta$ nor to $\bar{\eta}$ and, thus, $a$ is not ordinal GR-dominant. QED 